\title{
PROOF OF THE ALTERNATING SIGN MATRIX CONJECTURE ${ }^{1}$
}

\author{
Doron ZEILBERGER ${ }^{2}$
}

Submitted: May 1, 1995; Accepted: July 25, 1995

Checked by ${ }^{3}$ : David Bressoud and

Gert Almkvist, Noga Alon, George Andrews, Anonymous, Dror Bar-Natan, Francois Bergeron, Nantel Bergeron, Gaurav Bhatnagar, Anders Björner, Jonathan Borwein, Mireille Bousquest-Mélou, Francesco Brenti, E. Rodney Canfield, William Chen, Chu Wenchang, Shaun Cooper, Kequan Ding, Charles Dunkl, Richard Ehrenborg, Leon Ehrenpreis, Shalosh B. Ekhad, Kimmo Eriksson, Dominique Foata, Omar Foda, Aviezri Fraenkel, Jane Friedman, Frank Garvan, George Gasper, Ron Graham, Andrew Granville, Eric Grinberg, Laurent Habsieger, Jim Haglund, Han Guo-Niu, Roger Howe, Warren Johnson, Gil Kalai, Viggo Kann, Marvin Knopp, Don Knuth, Christian Krattenthaler, Gilbert Labelle, Jacques Labelle, Jane Legrange, Pierre Leroux, Ethan Lewis, Daniel Loeb, John Majewicz, Steve Milne, John Noonan, Kathy O'Hara, Soichi Okada, Craig Orr, Sheldon Parnes, Peter Paule, Bob Proctor, Arun Ram, Marge Readdy, Amitai Regev, Jeff Remmel, Christoph Reutenauer, Bruce Reznick, Dave Robbins, Gian-Carlo Rota, Cecil Rousseau, Bruce Sagan, Bruno Salvy, Isabella Sheftel, Rodica Simion, R. Jamie Simpson, Richard Stanley, Dennis Stanton, Volker Strehl, Walt Stromquist, Bob Sulanke, X.Y. Sun, Sheila Sundaram, Raphaële Supper, Nobuki Takayama, Xavier G. Viennot, Michelle Wachs, Michael Werman, Herb Wilf, Celia Zeilberger, Hadas Zeilberger, Tamar Zeilberger, Li Zhang, Paul Zimmermann .

\section{Dedicated to my Friend, Mentor, and Guru, Dominique Foata.}

Two stones build two houses. Three build six houses. Four build four and twenty houses. Five build hundred and twenty houses. Six build Seven hundreds and twenty houses. Seven build five thousands and forty houses. From now on, [exit and] ponder what the mouth cannot speak and the ear cannot hear.

(Sepher Yetsira IV,12)

\begin{abstract}
The number of $n \times n$ matrices whose entries are either $-1,0$, or 1 , whose row- and column- sums are all 1 , and such that in every row and every column the non-zero entries alternate in sign, is proved to be $[1 ! 4 ! \ldots(3 n-2) !] /[n !(n+1) ! \ldots(2 n-1) !]$, as conjectured by Mills, Robbins, and Rumsey.

1 original version written December 1992. The Maple package ROBBINS accompanying this paper, can be downloaded from the www address in footnote 2 below.

2 Department of Mathematics, Temple University, Philadelphia, PA 19122, USA.

E-mail:zeilberg@math.temple.edu. WWW:http://www.math.temple.edu/ zeilberg. Supported in part by the NSF.

3 See the Exodion for affiliations, attribution, and short bios.
\end{abstract}




\section{INTRODUCTION}

The number of permutations ("houses") that can be made using $n$ objects ("stones"), for $n \leq 7$, is given in Sepher Yetsira (Ch. IV, v. 12), a Cabalistic text written more than 1700 years ago. The general formula, $n$ !, was stated and proved about 1000 years later by Rabbi Levi Ben Gerson ("Ralbag"). The Cabala, which is a combinatorial Theory Of Everything (both physical and spiritual), was interested in this problem because $n$ ! is the number of inverse images $\tau^{-1}(w)$ of a generic $n$-lettered word $w$ under the canonical homomorphism $\tau$ :

$\tau:\{\aleph, \text { bet }, \ldots, \text { shin }, \text { tav }\}^{*} \rightarrow\{\aleph, \ldots, \text { tav }\}^{*} /\{\aleph($ bet $)=($ bet $) \aleph, \ldots,($ shin $)($ tav $)=($ tav $)($ shin $)\} \quad$.

The homomorphism $\tau$ is of considerable interest, since two words $w_{1}$, and $w_{2}$ are temura-equivalent (anagrams) if $\tau\left(w_{1}\right)=\tau\left(w_{2}\right)$.

A coarser, but just as important, equivalence relation on Hebrew words and sentences is the one induced by the homomorphism

$$
G:\{\aleph, \text { bet }, \ldots, \operatorname{shin}, \operatorname{tav}\}^{*} \rightarrow \mathcal{N} \quad
$$

defined by $G(\aleph)=1, G($ bet $)=2, \ldots, G($ shin $)=300, G($ tav $)=400$, and extended homomorphically. Two words $w_{1}, w_{2}$ are said to be Gematrically equivalent if $G\left(w_{1}\right)=G\left(w_{2}\right)$.

Modern-day numerologists, like Mills, Robbins, and Rumsey, use a different kind of Gematria, one that searches for equality between sequences of cardinalities of combinatorial families. This enabled them to conjecture a series of remarkable and tantalizing enumeration identities[MRR1-3], [Stanl]. Most of these conjectures concern a natural generalization of the notion of permutation, called alternating sign matrix.

A permutation $\pi$ may be described in terms of its corresponding permutation matrix, that is the $0-1$ matrix obtained by making the $i^{\text {th }}$ row have all zeros except for a 1 at the $\pi(i)^{t h}$ column. What emerges is a $0-1$ matrix whose row- and column- sums are all 1 .

Since 3 is the cardinality of the set $\{\aleph$, mem, shin $\}$, the "mother-letters", dear to the authors of Sepher Yetsira, they would have most likely enthusiastically approved of the generalization of permutation matrices, alternating sign matrices, introduced by Robbins and Rumsey[RR] in their study of a determinant-evaluation rule due to yet another wizard, the Rev. Charles Dodgson. Rather than use only the two symbols 0,1 as entries, an alternating sign matrix is allowed the use of the three symbols $\{-1,1,0\}$ (corresponding to guilt, innocence, and the tongue of the law, respectively). The row- and column- sums have still to be 1 , and in addition, in every row and every column, the non-zero elements, $1,-1$ (right and wrong), have to alternate.

Mills, Robbins, and Rumsey[MRR1] discovered that, like their predecessors the permutations, that are enumerated by the beautiful formula $n$ !, these new mysterious objects seem to be enumerated by an almost equally simple formula:

$$
A_{n}:=\prod_{i=0}^{n-1} \frac{(3 i+1) !}{(n+i) !}
$$


the now famous[R][Z3] sequence 1,2,7,42,429,7436 .., first encountered by George Andrews[A1].

In this paper I present the first proof of this fact. I do hope that this is not the last proof, and that a shorter, more direct, elegant, and combinatorial proof will be found one day. Meanwhile, this paper should relieve at least some of Dave Robbins's mathschmerz, expressed so eloquently in [R]:

These conjectures are of such compelling simplicity that it is hard to understand how any mathematician can bear the pain of living without understanding why they are true.

Much more serious than not knowing whether a given fact is true, is the agony of realizing that our cherished tools of the trade are inadequate to tackle a given problem. The fact that a conjecture resists vigorous attacks by skilled practitioners is an impetus for us either to sharpen our existing tools, or else create new ones. The value of a proof of an outstanding conjecture should be judged, not by its cleverness and elegance, and not even by its "explanatory power", but by the extent in which it enlarges our toolbox. By this standard, the present proof is adequate. Like most new tools, the present method of proof is a judicious assembly of existing tools, which I will now describe.

The first ingredient consists of partial recurrence equations (alias partial difference equations) and operators. The calculus of finite differences was introduced in the last century by discrete mathematician George Boole, but in this century was taken up, and almost monopolized by, continuous number crunchers, who called them finite difference schemes. A notable exception was Dick Duffin (e.g. [D]) through whose writings I learnt about these objects, and immediately fell in love with them (e.g. [Z1][Z2]). It was fun returning to my first love. ${ }^{4}$

Conspicuously missing from the present paper is my second love, bijective proofs (e.g. [ZB]), that were taught to me by Dominique Foata, Xavier G. Viennot, Herb Wilf and many others. However, doing bijections made me a better mathematician and person, so their implicit impact is considerable.

The second ingredient is my third love, constant term identities introduced to me by Dick Askey. Dennis Stanton[Stant] and John Stembridge[Ste] showed me how to crack them[Z4][Z5]. The Stanton-Stembridge trick (see below) was indeed crucial.

The third and last ingredient, which is not mentioned explicitly, but without which this proof could never have come to be, is my current love: computer algebra and Maple. Practically every lemma, sublemma, subsublemma ..., was first conjectured with the aid of Maple, and then tested by it. A Maple package, ROBBINS, that empirically (and in a few cases rigorously,) checks every non-trivial fact proved in this paper, is given as a companion to this paper, and should be used in conjunction with it. Almost every statement is followed by a reference to the procedure in ROBBINS that empirically corroborates it. [These are enclosed in square brackets; For example to see all alternating sign matrices of size 4, type $\operatorname{ASM}(4):$.]

4 "Mathematicians, like Proust and everyone else, are at their best when writing about their first love"- Gian-Carlo Rota (in: 'Discrete Thoughts', p. 3). 
Once you have downloaded ROBBINS to your favorite directory, get into Maple by typing maple $<\mathrm{CR}>$. Once in Maple, type read ROBBINS:, and follow the instructions given there. (By the way ezra means 'help' in Hebrew.) No knowledge of Maple is required, except for the fact that every command must end with a colon or semi-colon. For example, typing S15(4): would verify sublemma 1.5 for $k=4$.

I wish to thank Shalosh B. Ekhad for its diligent computations, and Russ de Flavia, our dedicated local UNIX guru, for his constant technical support.

This paper would have been little more than a curiosity if not for George Andrews's[A2] recent brilliant proof of another conjecture of Mills, Robbins, and Rumsey[MRR3] (conj. 2 of [Stanl]), that the number of so-called Totally Symmetric, Self- Complementary Plane Partitions (TSSCPP) is also given by $A_{n}$. All that I show is, that the sequence enumerating ASMs is the same as the one enumerating TSSCPPs, and then I take a free ride on Andrews's[A2] result that the latter is indeed $\{1,2,7,42,429, \ldots\}$.

This paper only settles the first, and simplest, conjecture, concerning the enumeration of alternating sign matrices. There are many variations and refinements listed in $[\mathrm{Stanl}][\mathrm{R}][\mathrm{MRR} 2,3]$. I am sure that the method of this paper should be capable of proving all of them. It is also possible that the present method of proof, combined with the multi-WZ method[WZ], could be used to prove a stronger conjecture of [MRR1,2](conj. 3 of [Stanl]), directly, in which case the present paper would also furnish an alternative proof of Andrews's[A2] TSSCPP theorem.

\section{A MORE GENERAL, AND HENCE EASIER, CONJECTURE}

The first step, already undertaken in [MRR2-3], is to find more congenial "data structures" for both alternating sign matrices, and for TSSCPPs. Alternating sign matrices of size $n$ are in easy bijection ([MRR2][R]) with monotone triangles. A monotone triangle is a triangular array of positive integers $a_{i, j}, 1 \leq i \leq n, 1 \leq j \leq n-i+1$, such that, $a_{i, j}<a_{i, j+1}$, and $a_{i, j} \leq a_{i+1, j} \leq a_{i, j+1}$. In addition we require that the first row is $1,2, \cdots, n$, i.e. $a_{1, j}=j$, for $j=1, \cdots, n$. We will rename them $n$-Gog triangles, and the creatures obtained by chopping off all entries with $j>k$, will be called $n \times k$-Gog trapezoids. An $n$-Gog triangle is an $n \times n$-Gog trapezoid. For example the following is one of the $429 \quad 5-$ Gog triangles (formerly called monotone triangles of size 5 .)

$\begin{array}{lllll}1 & 2 & 3 & 4 & 5 \\ 1 & 3 & 4 & 5 & \\ 2 & 4 & 5 & & \\ 3 & 5 & & & \\ 4 & & & & \end{array}$.

[In order to view all of them type 'GOG(5,5):' in ROBBINS.] Retaining only the first three columns of the above triangle, yields one of the $3875 \times 3$-Gog trapezoids:

$\begin{array}{lll}1 & 2 & 3 \\ 1 & 3 & 4 \\ 2 & 4 & 5 \\ 3 & 5 & \\ 4 & & \end{array}$.


[In order to view all of them type 'GOG(3,5):' in ROBBINS.]

On the TSSCPP side, it was shown in [MRR3] that TSSCPPs whose 3D Ferrers graphs lie in the cube $[0,2 n]^{3}$ are in trivial bijection with triangular arrays $c_{i, j}, 1 \leq i \leq n, 1 \leq j \leq n-i+1$, of integers such that: (i) $1 \leq c_{i, j} \leq j$, (ii) $c_{i, j} \geq c_{i+1, j}$, and (iii) $c_{i, j} \leq c_{i, j+1}$. We will call such triangles $n$-Magog triangles, and the corresponding chopped variety, with exactly the same conditions as above, but $c_{i, j}$ is only defined for $1 \leq i \leq k$ rather than for $1 \leq i \leq n, n \times k$-Magog trapezoids. For example the following is one of the $429 \quad 5$-Magog triangles:

$\begin{array}{lllll}1 & 2 & 3 & 3 & 5 \\ 1 & 2 & 2 & 3 & \\ 1 & 2 & 2 & & \\ 1 & 2 & & & \\ 1 & & & & \end{array}$.

[In order to view all of them type 'MAGOG(5,5):' in ROBBINS.] Retaining only the first three rows of the above Magog-triangle, yields one of the $3875 \times 3$-Magog trapezoids:

$\begin{array}{lllll}1 & 2 & 3 & 3 & 5 \\ 1 & 2 & 2 & 3 & \\ 1 & 2 & 2 & & \end{array}$.

[ In order to view all of them type 'MAGOG(3,5):' in ROBBINS.]

Our goal is to prove the following statement, conjectured in [MRR3], and proved there for $k=2$.

Lemma 1: For $n \geq k \geq 1$, the number of $n \times k$-Gog trapezoids equals the number of $n \times k$-Magog trapezoids.

[ The number of $n$ by $k$ Magog trapezoids, for specific $n$ and $k$, is obtained by typing $b(k, n)$; while the number of $\mathrm{n}$ by $\mathrm{k}$ Gog trapezoids is given by $\mathrm{m}(\mathrm{k}, \mathrm{n})$; . To verify lemma 1 , type $\mathrm{S} 1(\mathrm{k}, \mathrm{n}):$. ]

This would imply, by setting $n=k$, that,

Corollary 1': For $n \geq 1$, the number of $n$-Gog triangles equals the number of $n$-Magog triangles.

Since $n$-Gog triangles are equi-numerous with $n \times n$ alternating sign matrices, and $n$-Magog triangles are equi-numerous with TSSCPPs bounded in $[0,2 n]^{3}$, this would imply, together with Andrews's[A2] affirmative resolution of the TSCCPP conjecture, the following result, that was conjectured in [MRR1].

The Alternating Sign Matrix Theorem: The number of $n \times n$ alternating sign matrices, for $n \geq 1$, is:

$$
\frac{1 ! 4 ! \ldots(3 n-2) !}{n !(n+1) ! \ldots(2 n-1) !}=\prod_{i=0}^{n-1} \frac{(3 i+1) !}{(n+i) !}
$$


The rest of this paper will consist of a proof of Lemma 1.

\section{NOMENCLATURE}

Throughout this paper, we will meet discrete functions $F\left(n ; a_{1}, \ldots, a_{k}\right)$ of $k+1$ discrete variables, $n, a_{1}, \ldots, a_{k}$, where $n \geq 0$, and $\left(a_{1}, \ldots, a_{k}\right)$ is confined to certain regions of discrete $k$-space $\mathcal{N}^{k}$, that depend on $n$. We will make extensive use of the shift operators $A_{i}, i=1, \ldots, k$, defined by:

$$
A_{i} F\left(a_{1}, \ldots, a_{i}, \ldots, a_{k}\right)=F\left(a_{1}, \ldots, a_{i}+1, \ldots, a_{k}\right) \quad .
$$

For any (positive or negative) integer $r$, we have:

$$
A_{i}^{r} F\left(a_{1}, \ldots, a_{i}, \ldots, a_{k}\right)=F\left(a_{1}, \ldots, a_{i}+r, \ldots, a_{k}\right)
$$

and more generally,

$$
A_{1}^{r_{1}} \ldots A_{i}^{r_{i}} \ldots A_{k}^{r_{k}} F\left(a_{1}, \ldots, a_{i}, \ldots, a_{k}\right)=F\left(a_{1}+r_{1}, \ldots, a_{i}+r_{i}, \ldots, a_{k}+r_{k}\right)
$$

We denote the identity operator by $I$. A partial linear recurrence operator (with constant coefficients) $P\left(A_{1}, \ldots, A_{k}\right)$ is any Laurent polynomial in the fundamental shift operators $A_{1}, \ldots, A_{k}$. For example

$$
\left(I-A_{1}^{-1}\right)\left(I-A_{2}^{-1}\right) F\left(a_{1}, a_{2}\right)=F\left(a_{1}, a_{2}\right)-F\left(a_{1}-1, a_{2}\right)-F\left(a_{1}, a_{2}-1\right)+F\left(a_{1}-1, a_{2}-1\right)
$$

We will also meet polynomials and rational functions $f\left(x_{1}, \ldots, x_{k}\right)$ that live on continuous $k$-space. The symmetric group $\mathcal{S}_{k}$ of permutations acts on $f\left(x_{1}, \ldots, x_{k}\right)$ as follows. For any permutation $\pi=[\pi(1), \ldots, \pi(k)]$, let $\pi f\left(x_{1}, \ldots, x_{k}\right)$ be the rational function obtained from $f\left(x_{1}, \ldots, x_{k}\right)$ by replacing $x_{i}$ by $x_{\pi(i)}$, for $i=1 \ldots k$. In symbols:

$$
\pi f\left(x_{1}, \ldots, x_{k}\right)=\left.f\left(x_{1}, \ldots, x_{k}\right)\right|_{\left\{x_{1} \rightarrow x_{\pi(1)}, \ldots, x_{k} \rightarrow x_{\pi(k)}\right\}} \quad,
$$

for example, $[2,3,1]\left(x_{1}+2 x_{2}+3 x_{3}\right)=x_{2}+2 x_{3}+3 x_{1}$.

Recall that the number of inversions, inv $\pi$, of a permutation $\pi \in S_{k}$ is the number of pairs $(i, j)$, with $1 \leq i<j \leq k$ such that $\pi(i)>\pi(j)$. Recall also that the sign of a permutation $\operatorname{sgn}(\pi)$ may be defined by $(-1)^{\operatorname{inv} \pi}$.

A rational function $f\left(x_{1}, \ldots, x_{k}\right)$ is called $\mathcal{S}_{k}$-antisymmetric, or antisymmetric for short, if for $i=1, \ldots, k-1$ :

$$
\left.f\left(x_{1}, \ldots, x_{k}\right)\right|_{x_{i} \leftrightarrow x_{i+1}}=-f\left(x_{1}, \ldots, x_{k}\right)
$$


or equivalently, since the transpositions $\{(i, i+1), 1 \leq i \leq k-1\}$ generate the symmetric group $\mathcal{S}_{k}$, the equality $: \pi f(x)=\operatorname{sgn}(\pi) f(x)$, holds for every $\pi \in \mathcal{S}_{k}$.

The group of signed permutations, that we will denote by $W\left(\mathcal{B}_{k}\right)$ (since it happens to be the Weyl group of the root system $\mathcal{B}_{k}$, but this is not (directly) relevant to our proof), consists of pairs $(\pi, \varepsilon)$, where $\pi \in \mathcal{S}_{k}$ and $\varepsilon$ is a sign-assignment: $\varepsilon=\left(\varepsilon_{1}, \ldots, \varepsilon_{k}\right)$, where the $\varepsilon_{i}$ are either +1 or -1 . A sign assignment $\varepsilon$ acts on $f\left(x_{1}, \ldots, x_{k}\right)$ by

$$
\varepsilon f(x)=f\left(\varepsilon_{1}\left(x_{1}\right), \ldots, \varepsilon_{k}\left(x_{k}\right)\right) \quad,
$$

where, for any variable $t$ :

$$
\varepsilon_{i}(t):=t \quad \text { if } \quad \varepsilon_{i}=1, \quad \text { and } \quad \varepsilon_{i}(t):=1-t \quad \text { if } \quad \varepsilon_{i}=-1 \quad .
$$

For example $[-1,-1,+1]\left(x_{1}^{2} x_{2}^{3} x_{3}^{4}\right)=\left(1-x_{1}\right)^{2}\left(1-x_{2}\right)^{3} x_{3}^{4}$.

A signed permutation $(\pi, \varepsilon)$ acts on $f\left(x_{1}, \ldots, x_{k}\right)$ in the following way:

$$
(\pi, \varepsilon) f(x)=\varepsilon \pi f(x)
$$

For example $([2,3,1],[+1,-1,-1])\left[x_{1}^{2}+2 x_{2}+x_{3}^{3}\right]=\left(1-x_{2}\right)^{2}+2\left(1-x_{3}\right)+x_{1}^{3}$.

Now it is time to define the sign of a signed permutation (no pun intended!), $g=(\pi, \varepsilon)$.

Definition SIGN: For any signed permutation $g=(\pi, \varepsilon)$, we define $\operatorname{sgn}(g):=\operatorname{sgn}(\pi) \operatorname{sgn}(\varepsilon)$, where $\operatorname{sgn}(\varepsilon)$ is $(-1)$ [number of -1 's].

Throughout this paper $\bar{t}:=1-t$. Since this notation will be used so frequently we will say it again, in bold face:

Crucial Notation $: \overline{\mathbf{t}}:=\mathbf{1}-\mathbf{t}$.

Warning: $\bar{t}$ Is Not Complex Conjugation!

A rational function $f\left(x_{1}, \ldots, x_{k}\right)$ is $W\left(\mathcal{B}_{k}\right)$-antisymmetric if it is $\mathcal{S}_{k}$-antisymmetric, and also

$$
f\left(\bar{x}_{1}, \ldots, x_{k}\right)=-f\left(x_{1}, \ldots, x_{k}\right) .
$$

Equivalently, $f\left(x_{1}, \ldots, x_{k}\right)$ is $W\left(\mathcal{B}_{k}\right)$-antisymmetric if for any signed-permutation $g \in W\left(\mathcal{B}_{k}\right)$, we have: $g f(x)=\operatorname{sgn}(g) f(x)$. This follows from the well-known (and easy) fact that the group $W\left(\mathcal{B}_{k}\right)$ is generated by the generators $(i, i+1)$ of the symmetric group, along with the 'sign change' $x_{1} \rightarrow \bar{x}_{1}$.

A Laurent formal power series $f\left(x_{1}, \ldots, x_{k}\right)$ is anything of the form

$$
f\left(x_{1}, \ldots, x_{k}\right)=\sum_{\alpha_{1} \geq L, \ldots, \alpha_{k} \geq L} a_{\alpha_{1}, \ldots, \alpha_{k}} x_{1}^{\alpha_{1}} \ldots x_{k}^{\alpha_{k}}
$$


where $L$ is a (non-positive) integer. If $L=0$ then it is a run-of-the-mill formal power series. If $a_{\alpha_{1}, \ldots, \alpha_{k}}$ is zero except for finitely many $\alpha$ 's then we have a Laurent polynomial. The constant term of a Laurent formal power series $f(x)=f\left(x_{1}, \ldots, x_{k}\right)$, denoted by $C T f(x)$, is the coefficient of $x_{1}^{0} \ldots x_{k}^{0}$, i.e. $a_{0,0, \ldots, 0,0}$.

\section{CRUCIAL FACTS}

Crucial Fact $\aleph_{0}$ : A rational function $f\left(x_{1}, \ldots, x_{k}\right)$ possesses a Laurent expansion if it is of the form

$$
f\left(x_{1}, \ldots, x_{k}\right)=\frac{P\left(x_{1}, \ldots, x_{k}\right)}{x_{1}^{\gamma_{1}} \ldots x_{k}^{\gamma_{k}} Q\left(x_{1}, \ldots, x_{k}\right)}
$$

where $P$ and $Q$ are polynomials in $\left(x_{1}, \ldots, x_{k}\right)$, and most importantly, $Q$ has a non-zero constant term, i.e. $Q(0,0, \ldots, 0) \neq 0$, and the $\gamma_{i}$ are (not necessarily positive) integers.

Proof: Let the constant term of $Q, Q(0,0, \ldots, 0)$, be denoted by $C$. Expanding, we get:

$\frac{1}{Q\left(x_{1}, \ldots, x_{k}\right)}=\left(C+\sum_{\alpha_{1}+\ldots+\alpha_{k}>0} q_{\alpha_{1}, \ldots, \alpha_{k}} x_{1}^{\alpha_{1}} \ldots x_{k}^{\alpha_{k}}\right)^{-1}=\sum_{l=0}^{\infty}(-1)^{l} \frac{\left(\sum_{\alpha_{1}+\ldots+\alpha_{k}>0} q_{\alpha_{1}, \ldots, \alpha_{k}} x_{1}^{\alpha_{1}} \ldots x_{k}^{\alpha_{k}}\right)^{l}}{C^{l+1}}$

The right side "converges" in the ring of formal power series (the coefficient of any fixed monomial gets contributions from only finitely many terms in the above sum). It follows that, under the condition on $Q, P / Q$ also possesses a formal power series, and dividing by the monomial $x_{1}^{\gamma_{1}} \ldots x_{k}^{\gamma_{k}}$ results in a formal Laurent series.

The condition $Q(0, \ldots, 0) \neq 0$ is necessary, since, for example, $1 /(x+y)$ does not have a formal Laurent series. (Try it!)

\section{Iterated Constant-Terming}

The constant term $C T_{x} f(x)$ of a rational function $f(x)$ of a single variable $x$, is defined to be the coefficient of $x^{0}$ in its Laurent expansion. This is well-defined, since such a rational function $f(x)$ of the single variable, $x$, can always be written in the form $P(x) /\left(x^{a} Q(x)\right)$, where $P(x)$ is a polynomial and $Q(x)$ is a polynomial with $Q(0) \neq 0$, and $a$ is a non-negative integer. By crucial fact $\aleph_{0}$, it possesses a genuine Laurent expansion, and $C T_{x} f(x)$ is always well-defined.

Consider now an arbitrary rational function $f\left(x_{1}, \ldots, x_{k}\right)$. It can be viewed as a rational function in the variable $x_{k}$, with coefficients that are rational functions of the other variables $\left(x_{1}, \ldots, x_{k-1}\right)$. It follows that $C T_{x_{k}} f\left(x_{1}, \ldots, x_{k}\right)$ is well-defined, and is a certain rational function of $\left(x_{1}, \ldots, x_{k-1}\right)$, Hence $C T_{x_{k-1}} C T_{x_{k}} f\left(x_{1}, \ldots, x_{k}\right)$ is well-defined, and is a certain rational function of $\left(x_{1}, \ldots, x_{k-2}\right)$ and so on, until we get that $C T_{x_{1}} C T_{x_{2}} \ldots C T_{x_{k}} f\left(x_{1}, \ldots, x_{k}\right)$, that we will abbreviate to $C T_{x_{1}, \ldots, x_{k}} f\left(x_{1}, \ldots, x_{k}\right)$, is well-defined and is a certain number. 
More formally we have the following recursive definition:

Definition ITERCT: Let $f\left(x_{1}, \ldots, x_{k}\right)$ be any rational function. For $r=1, \ldots, k, C T_{x_{1}, \ldots, x_{r}} f\left(x_{1}, \ldots, x_{k}\right)$ is equal to $C T_{x_{1}} f\left(x_{1}, \ldots, x_{k}\right)$ if $r=1$, and otherwise

$$
C T_{x_{1}, \ldots, x_{r}} f\left(x_{1}, \ldots, x_{k}\right):=C T_{x_{1}, \ldots, x_{r-1}}\left[C T_{x_{r}} f\left(x_{1}, \ldots, x_{k}\right)\right]
$$

Crucial Fact $\aleph_{1}$ : Let $f\left(x_{1}, \ldots, x_{k}\right)$ be a rational function that possesses a Laurent formal power series, and that is antisymmetric w.r.t. two variables $x_{i}, x_{j}$ (i.e. $\left.f\right|_{x_{i} \leftrightarrow x_{j}}=-f$.) Then $C T_{x_{1}, \ldots, x_{k}} f\left(x_{1}, \ldots, x_{k}\right)=0$.

Crucial Fact $\aleph_{2}$ : Let $P(x)$ be a polynomial of degree $\leq 2 A$, in (the single variable) $x$, that is anti-symmetric w.r.t. to the operation $x \rightarrow 1-x$ (i.e. $P(1-x)=-P(x)$ ). Then

$$
C T_{x}\left[\frac{P(x)}{(1-x)^{A+1} x^{A}}\right]=0 .
$$

Proof: Immediate from crucial fact $\aleph_{5}$ below.

Crucial Fact $\aleph_{3}$ : Let $f\left(x_{1}, \ldots, x_{k}\right)$ be a rational function, and define a discrete function $F\left(a_{1}, \ldots, a_{k}\right)$ by

$$
F\left(a_{1}, \ldots, a_{k}\right):=C T_{x_{1}, \ldots, x_{k}} \frac{f\left(x_{1}, \ldots, x_{k}\right)}{x_{1}^{a_{1}} \ldots x_{k}^{a_{k}}}
$$

Then for any Laurent polynomial $P\left(x_{1}, \ldots, x_{k}\right)$, we have

$$
P\left(A_{1}^{-1}, \ldots, A_{k}^{-1}\right) F\left(a_{1}, \ldots, a_{k}\right)=C T_{x_{1}, \ldots, x_{k}} \frac{P\left(x_{1}, \ldots, x_{k}\right) f\left(x_{1}, \ldots, x_{k}\right)}{x_{1}^{a_{1}} \ldots x_{k}^{a_{k}}}
$$

Proof: This is obvious if $P$ is a monomial. Since a polynomial is a linear combination of monomials, and both sides are linear in $P$, it is also clear in general.

Crucial Fact $\aleph_{4}$ (The Stanton-Stembridge trick): For any permutation $\pi \in \mathcal{S}_{k}$ and any rational function that possesses a formal Laurent series, $f(x)=f\left(x_{1}, \ldots, x_{k}\right)$, we have

$$
C T_{x_{1}, \ldots, x_{k}}[\pi f(x)]=C T_{x_{1}, \ldots, x_{k}} f(x)
$$

Equivalently: 


$$
C T_{x_{\pi(1)}, \ldots, x_{\pi(k)}} f(x)=C T_{x_{1}, \ldots, x_{k}} f(x)
$$

Proof: Applying $\pi$ amounts to renaming the variables. However, the constant term is obviously unaffected by this renaming.

Warning: Crucial fact $\aleph_{4}$ is false if the rational function $f$ does not possess a Laurent series, for example when $f\left(x_{1}, x_{2}\right)=x_{1} /\left(x_{1}+x_{2}\right)$.

Crucial Fact $\aleph_{5}$ : Let $A$ be a non-negative integer, and let $P(x)$ be a Laurent polynomial in $x$ of degree $\leq 2 A$, then

$$
C T_{x}\left[\frac{P(x)}{(1-x)^{A+1} x^{A}}\right]=C T_{x}\left[\frac{P(1-x)}{(1-x)^{A+1} x^{A}}\right] .
$$

Proof: $P(x)$ is a linear combination of powers $x^{b}$, for $b \leq 2 A$. If $P(x)=x^{b}$, with $A<b \leq 2 A$, both sides vanish, while for $P(x)=x^{b}$, with $b \leq A$, we have

$$
\begin{aligned}
& C T_{x}\left[\frac{x^{b}}{(1-x)^{A+1} x^{A}}\right]=C T_{x}\left[\frac{1}{(1-x)^{A+1} x^{A-b}}\right]=\left(\begin{array}{c}
2 A-b \\
A-b
\end{array}\right) \\
= & \left(\begin{array}{c}
2 A-b \\
A
\end{array}\right)=C T_{x}\left[\frac{1}{(1-x)^{A-b+1} x^{A}}\right]=C T_{x}\left[\frac{(1-x)^{b}}{(1-x)^{A+1} x^{A}}\right] .
\end{aligned}
$$

We also need the celebrated

Vandermonde's Determinant Identity:

$$
\sum_{\pi \in \mathcal{S}_{k}} \operatorname{sgn}(\pi) \cdot \pi\left(\prod_{i=1}^{k} x_{i}^{i-1}\right)=\prod_{1 \leq i<j \leq k}\left(x_{j}-x_{i}\right)
$$

Proof: View both sides as polynomials in $x_{1}$ of degree $k-1$. Both sides agree (and in fact are zero) at $x_{1}=x_{2}, \ldots, x_{1}=x_{k}$, and they also agree at $x_{1}=0$ by induction on $k$. Since they agree at $k$ distinct values, they agree everywhere.

A longer, but much nicer, proof (that turned out to be seminal[ZB]) was given by Ira Gessel[G].

\section{Iterated Residues}

Later, we will find it necessary to convert our constant-term expressions to "residue" expressions. People who, like myself, (and John Riordan), are horrified by analysis, need not worry. The residue, $\operatorname{Res}_{x} f(x)$, of a rational function of a single variable $x$, is defined as the coefficient of $x^{-1}$ in the (formal) Laurent expansion of $f(x)$. It is well-defined for the same reason that $C T_{x} f(x)$ is: a function of a single variable always possesses a Laurent series, thanks to crucial fact $\aleph_{0}$. 
The iterated residue $\operatorname{Res}_{x_{1}, \ldots, x_{k}} f$ is defined to be $\operatorname{Res}_{x_{1}}\left[\operatorname{Res}_{x_{2}}\left[\ldots\left[\operatorname{Res}_{x_{k}} f\right] \ldots\right]\right]$. [Try out IterRes in ROBBINS].

Trivial (Yet Crucial) Fact $\aleph_{6}$ :

$$
C T_{x_{1}, \ldots, x_{k}} f\left(x_{1}, \ldots, x_{k}\right)=\operatorname{Res}_{x_{1}, \ldots, x_{k}}\left[\frac{f\left(x_{1}, \ldots, x_{k}\right)}{x_{1} \ldots x_{k}}\right] .
$$

For future reference, we need to recast crucial facts $\aleph_{4}$ and $\aleph_{5}$ in terms of residues.

Crucial Fact $\aleph_{4}^{\prime}$ (The Stanton-Stembridge trick): For any permutation $\pi \in \mathcal{S}_{k}$ and any rational function that possesses a formal Laurent series, $f(x)=f\left(x_{1}, \ldots, x_{k}\right)$, we have

$$
\operatorname{Res}_{x_{1}, \ldots, x_{k}}[\pi f(x)]=\operatorname{Res}_{x_{1}, \ldots, x_{k}} f(x)
$$

Equivalently:

$$
\operatorname{Res}_{x_{\pi(1)}, \ldots, x_{\pi(k)}} f(x)=\operatorname{Res}_{x_{1}, \ldots, x_{k}} f(x)
$$

Crucial Fact $\aleph_{5}^{\prime}$ : Let $A$ be a non-negative integer, and let $P(x)$ be a Laurent polynomial in $x$ of degree $\leq 2 A$, then

$$
\operatorname{Res}_{x}\left[\frac{P(x)}{(1-x)^{A+1} x^{A+1}}\right]=\operatorname{Res}_{x}\left[\frac{P(1-x)}{(1-x)^{A+1} x^{A+1}}\right] .
$$

\section{antisymmetrizers}

Crucial Fact $\aleph_{7}$ : For any rational function $f\left(x_{1}, \ldots, x_{k}\right)$, its antisymmetrizer w.r.t. $\mathcal{S}_{k}$ :

$$
\sum_{\pi \in \mathcal{S}_{k}} \operatorname{sgn}(\pi) \cdot \pi f\left(x_{1}, \ldots, x_{k}\right)
$$

is an $\mathcal{S}_{k^{-}}$antisymmetric function.

[ Try antisymmetrizerS_k in ROBBINS].

Crucial Fact $\aleph_{7}^{\prime}$ : For any rational function $f\left(x_{1}, \ldots, x_{k}\right)$, its antisymmetrizer w.r.t. $W\left(\mathcal{B}_{k}\right)$ :

$$
\sum_{g \in W\left(\mathcal{B}_{k}\right)} \operatorname{sgn}(g) \cdot g f\left(x_{1}, \ldots, x_{k}\right)
$$

is a $W\left(\mathcal{B}_{k}\right)$-anti-symmetric function.

[ Try antisymmetrizerWB_k in ROBBINS].

Crucial Fact $\aleph_{8}$ : Any $S_{k}-$ antisymmetric polynomial is divisible by 


$$
\prod_{1 \leq i<j \leq k}\left(x_{j}-x_{i}\right)
$$

Proof: Let's view the polynomial as a polynomial in $x_{1}$. By anti-symmetry, it vanishes at $x_{1}=x_{i}$, for $i=2,3, \ldots, k$, hence is divisible by $\left(x_{1}-x_{2}\right) \cdots\left(x_{1}-x_{n}\right)$. Similarly (or by (anti-)symmetry) it is also divisible by all $\left(x_{i}-x_{j}\right), 2 \leq i<j \leq k$, and hence by their product.

Crucial Fact $\aleph_{8}^{\prime}$ : Any $W\left(\mathcal{B}_{k}\right)-$ antisymmetric polynomial is divisible by

$$
\Delta_{k}\left(x_{1}, \ldots, x_{k}\right):=\prod_{i=1}^{k}\left(1-2 x_{i}\right) \prod_{1 \leq i<j \leq k}\left(x_{j}-x_{i}\right)\left(x_{j}+x_{i}-1\right)
$$

Proof: Let's view the polynomial as a polynomial in $x_{1}$. By $W\left(\mathcal{B}_{k}\right)$-antisymmetry, it vanishes at $x_{1}=x_{i}$, for $i=2,3, \ldots, k$, as well as at $x_{1}=\bar{x}_{i}$, and at $x_{1}=1 / 2$. Hence it is divisible by

$$
\left(1-2 x_{1}\right) \prod_{j=2}^{k}\left(x_{1}-x_{j}\right)\left(x_{1}+x_{j}-1\right)
$$

Similarly, (or by (anti-)symmetry) it is divisible by all the other factors of $\Delta_{k}$, and hence by $\Delta_{k}$ itself. 


\section{Act I. COUNTING MAGOG}

And I will send a fire on Magog... and they shall know that I am the Lord.

(Ezekiel XXXIX,6)

Recall that an $n \times k$-Magog trapezoid, where $n \geq k \geq 1$, is a trapezoidal array of integers $\left(c_{i, j}\right)$, $1 \leq i \leq k, 1 \leq j \leq n-i+1$, such that:

$$
\text { (i) } 1 \leq c_{i, j} \leq j \quad, \quad(i i) \quad c_{i, j} \geq c_{i+1, j} \quad, \quad \text { (iii) } \quad c_{i, j} \leq c_{i, j+1}
$$

Sublemma 1.1: The total number of $n \times k-$ Magog trapezoids, let's call it $b_{k}(n)$, is given by the following constant term expression:

$$
b_{k}(n)=C T_{x_{1}, \ldots, x_{k}}\left\{\frac{\Delta_{k}\left(x_{1}, \ldots, x_{k}\right)}{\prod_{i=1}^{k} x_{i}^{n+k-i-1}\left(\bar{x}_{i}\right)^{n+k+1} \prod_{1 \leq i<j \leq k}\left(1-x_{i} x_{j}\right)}\right\},
$$

where,

$$
\Delta_{k}\left(x_{1}, \ldots, x_{k}\right):=\prod_{i=1}^{k}\left(1-2 x_{i}\right) \prod_{1 \leq i<j \leq k}\left(x_{j}-x_{i}\right)\left(x_{j}+x_{i}-1\right)
$$

[ Type 'S11 $(k, n)$ :' in ROBBINS, for specific k,n.]

Proof: Let $B_{k}\left(n ; a_{1}, \ldots, a_{k}\right)$ be the number of $n \times k$ Magog trapezoids $\left(c_{i, j}\right)$, such that for $i=$ $1, \ldots, k, c_{i, n-i+1}=a_{i}$. In other words, the rightmost border is prescribed by the $a_{i}$. By the definition of Magog trapezoids, the natural domain of the $(k+1)$-variable discrete function $B_{k}(-;-, \ldots,-)$ is

$$
\begin{gathered}
\operatorname{Land\_ Of\_ Magog} k_{k}:= \\
\left\{\left(n ; a_{1}, \ldots, a_{k}\right) \mid n \geq k, \quad n \geq a_{1} \geq a_{2} \geq \ldots \geq a_{k} \geq 1, \quad \text { and } a_{i} \leq n-i+1 \quad \text { for } i=1, \ldots, k\right\} .
\end{gathered}
$$

It is convenient to extend this region to the larger region

$$
\left\{\left(n ; a_{1}, \ldots, a_{k}\right) \mid n \geq k, \quad n \geq a_{1} \geq a_{2} \geq \ldots \geq a_{k} \geq 1\right\}
$$

and to define $B_{k}\left(n ; a_{1}, \ldots, a_{k}\right)$ to be 0 whenever $a_{i}>n-i+1$ for one or more $i(1 \leq i \leq k)$. This makes perfect combinatorial sense, since the number of Magog-trapezoids with such $a_{i}$ 's, that break the rules, is 0 .

The following subsublemma gives a constant term expression for $B_{k}$.

Subsublemma 1.1.1: Let

$$
C_{k}\left(n ; a_{1}, \ldots, a_{k}\right):=C T_{x_{1}, \ldots, x_{k}}\left\{\frac{\Delta_{k}\left(x_{1}, \ldots, x_{k}\right)}{\prod_{i=1}^{k} x_{i}^{a_{i}+k-i-1}\left(\bar{x}_{i}\right)^{k+n}}\right\},
$$


where $\Delta_{k}$ is defined in Eq. (Delta) in the statement of sublemma 1.1 above. For $n \geq k \geq 1$ and for all $\left(n ; a_{1}, \ldots, a_{k}\right)$ for which $n \geq a_{1} \geq \ldots \geq a_{k} \geq 1$, we have:

$$
B_{k}\left(n ; a_{1}, \ldots, a_{k}\right)=C_{k}\left(n ; a_{1}, \ldots, a_{k}\right)
$$

[ Type 'S111 $(\mathrm{k}, \mathrm{n})$ :' in ROBBINS, for specific k,n.]

Proof: It is convenient to extend $B_{k}$ even further to the following larger domain:

$$
\begin{aligned}
& \overline{{\operatorname{Land} \_O f \_M a g o g_{k}}_{1}}:= \\
& \left\{\left(n ; a_{1}, \ldots, a_{k}\right) \mid n \geq k \geq 1, \quad n-a_{1} \geq-1, a_{1}-a_{2} \geq-1, \ldots, a_{k-1}-a_{k} \geq-1, a_{k} \geq 0 \quad\right\} \quad
\end{aligned}
$$

and to define $B_{k}$ to be zero in the "no-man's-land" points that are in $\overline{\operatorname{Land}_{-} O f \_M a g o g_{k}}$ but not in Land_Of_Magog L $_{-}$.

We will prove that subsublemma 1.1.1 holds in this larger domain $\overline{\operatorname{Land\_ Of\_ Magog}_{k}}$. This would follow from the following three subsubsublemmas.

Subsubsublemma 1.1.1.1: Let $k \geq 1$. The following partial recurrence holds in the subset of $\overline{\operatorname{Land}_{-} O f_{-} \operatorname{Magog}_{k}}$ of points $\left(n ; a_{1}, \ldots, a_{k}\right)$ for which $n>k$, and $n \geq a_{1} \geq a_{2} \geq \ldots \geq a_{k} \geq 1$ :

$$
\left\{\prod_{i=1}^{k}\left(I-A_{i}^{-1}\right)\right\} B_{k}\left(n ; a_{1}, \ldots, a_{k}\right)=B_{k}\left(n-1 ; a_{1}, \ldots, a_{k}\right) .
$$

$B_{k}\left(n ; a_{1}, \ldots, a_{k}\right) \quad$ also satisfies the following initial/boundary conditions in $\left(n ; a_{1}, \ldots, a_{k}\right) \in \overline{\text {Land_Of_Magog }_{k}}$ :

For $i=1,2, \ldots, k-1$,

$$
B_{k}\left(n ; a_{1}, \ldots, a_{k}\right)=0 \quad, \quad \text { on } \quad a_{i}-a_{i+1}=-1
$$

Also:

$$
B_{k}\left(n ; a_{1}, \ldots, a_{k}\right)=0 \quad, \quad \text { on } \quad a_{k}=0
$$

Furthermore:

$$
\begin{gathered}
B_{k}\left(n ; n+1, a_{2}, \ldots, a_{k}\right)=0, \text { and } \\
B_{k}\left(k ; a_{1}, \ldots, a_{k-1}, a_{k}\right)=B_{k-1}\left(k ; a_{1}, \ldots, a_{k-1}\right) \delta\left(a_{k}, 1\right)
\end{gathered}
$$

where, as usual, $\delta(i, j)$ is 1 or 0 according to whether $i=j$ is true or false respectively. Finally, $B_{k}(n ;-)$ satisfies the "Eve" condition:

$$
B_{1}\left(1 ; a_{1}\right)=\delta\left(a_{1}, 1\right), \quad\left(1 ; a_{1}\right) \in \overline{\operatorname{Land\_ Of\_ Magog}_{1}}
$$

[ Type 'S1111all $(\mathrm{k}, \mathrm{n})$ :' in ROBBINS, for specific $\mathrm{k}, \mathrm{n}$. 
Proof: Let's try to compute $B_{k}(n ;-)$ from $B_{k}(n-1 ;-)$. By looking at what vectors $\left(b_{1}, \ldots, b_{k}\right)$ can occupy the diagonal immediately to the left of the rightmost one, $c_{i, n-i}=b_{i}$, say, we get a

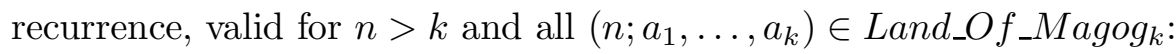

$$
B_{k}\left(n ; a_{1}, \ldots, a_{k}\right)=\sum_{\left(b_{1}, \ldots, b_{k}\right)} B_{k}\left(n-1 ; b_{1}, \ldots, b_{k}\right) .
$$

The summation extends over all the $b=\left(b_{1}, \ldots, b_{k}\right)$ such that $a_{i+1} \leq b_{i} \leq \min \left(a_{i}, n-i\right), i=$ $1 \ldots k-1, b_{k} \leq \min \left(a_{k}, n-k\right)$. Note that these conditions imply that $n-1 \geq b_{1} \geq b_{2} \ldots \geq b_{k}$.

(Ekhad) can be used to compile a table of $B_{k}$, together with the initial condition

$$
B_{k}\left(k ; a_{1}, \ldots, a_{k-1}, 1\right)=B_{k-1}\left(k ; a_{1}, \ldots, a_{k-1}\right),
$$

that holds because when $n=k$, we have a Magog triangle, and for it necessarily $c_{k, 1}=1$ and there is a one-one correspondence between $k \times k$ Magog triangles and $k \times(k-1)$ Magog-trapezoids obtained by deleting that $c_{k, 1}$ entry (or putting it back, if one wishes to go the other way.) This enables us to go down in the $k$-ladder until we reach $k=1$ for which $B_{1}(1 ; 1)=1$.

We can extend the set of $\left(n-1 ; b_{1}, \ldots, b_{k}\right)$ over which the summation in (Ekhad) takes place, so that $(E k h a d)$ becomes $\left(E k h a d^{\prime}\right)$ :

$$
B_{k}\left(n ; a_{1}, \ldots, a_{k}\right)=\sum_{a_{i+1} \leq b_{i} \leq a_{i}(1 \leq i \leq k)} B_{k}\left(n-1 ; b_{1}, b_{2}, \ldots, b_{k}\right)
$$

(where we put $a_{k+1}=0$,) since, whenever $b_{i}>n-i$, the contribution is zero, by our extended definition of $B_{k}$. The next thing to observe is that $\left(E k h a d^{\prime}\right)$ holds not only for $\left(n ; a_{1}, \ldots, a_{k}\right) \in$ Land_Of_Magog $f_{k}$ but for all points for which $n \geq a_{1} \geq \ldots \geq a_{k} \geq 1$, since for these extra points, the left side is 0 by the extended definition of $B_{k}$, and all the terms on the right side are also 0 for the same reason. Now,

$$
\begin{gathered}
\left\{\left(n-1 ; b_{1}, \ldots, b_{k}\right) \mid a_{2} \leq b_{1} \leq a_{1}, a_{3} \leq b_{2} \leq a_{2}, \ldots, a_{k} \leq b_{k-1} \leq a_{k-1}, b_{k} \leq a_{k}\right\} \\
-\left\{\left(n-1 ; b_{1}, \ldots, b_{k}\right) \mid a_{2} \leq b_{1} \leq\left(a_{1}-1\right), a_{3} \leq b_{2} \leq a_{2}, \ldots, a_{k} \leq b_{k-1} \leq a_{k-1}, b_{k} \leq a_{k}\right\} \\
=\left\{\left(n-1 ; a_{1}, b_{2}, \ldots, b_{k}\right) \mid a_{3} \leq b_{2} \leq a_{2}, \ldots, a_{k} \leq b_{k-1} \leq a_{k-1}, b_{k} \leq a_{k}\right\} .
\end{gathered}
$$

Using $\left(E k h a d^{\prime}\right)$, we get that for all $n \geq a_{1} \geq \ldots \geq a_{k} \geq 1$ :

$$
\left(I-A_{1}^{-1}\right) B_{k}\left(n ; a_{1}, \ldots, a_{k}\right)=\sum_{a_{i+1} \leq b_{i} \leq a_{i}(2 \leq i \leq k)} B_{k}\left(n-1 ; a_{1}, b_{2}, \ldots, b_{k}\right),
$$

(with the convention that $a_{k+1}=0$, ) for $\left(n ; a_{1}, \ldots, a_{k}\right)$ for which $n>k$ and $n \geq a_{1} \geq a_{2} \geq \ldots \geq$ $a_{k} \geq 1$.

Iterating the process, we get, in turn, that

$$
\left(I-A_{1}^{-1}\right) \ldots\left(I-A_{l}^{-1}\right) B_{k}\left(n ; a_{1}, \ldots, a_{k}\right)=\sum_{a_{i+1} \leq b_{i} \leq a_{i}(l+1 \leq i \leq k)} B_{k}\left(n-1 ; a_{1}, \ldots, a_{l}, b_{l+1}, \ldots, b_{k}\right)
$$


for $l=2, \ldots, k$. The final $l=k$ is $\left(P \Delta E\left(B_{k}\right)\right)$.

The boundary/initial conditions (B1)-(B2) follow from the fact that $B_{k}$ is defined to be 0 in the 'boundary' $\overline{\operatorname{Land}_{-} O f \_M a g o g_{k}} \backslash$ Land_Of_Magog ${ }_{k}$, while (B3) and (B4) follow straight from the (combinatorial) definition of $B_{k}$. This completes the proof of $(s u b)^{3}$ lemma 1.1.1.1.

Subsubsublemma 1.1.1.2: Let $C_{k}\left(n: a_{1}, \ldots, a_{k}\right)$ be the constant term expression defined in the statement of subsublemma 1.1.1. The following partial recurrence holds in the subset of $\overline{\text { Land_Of_Magog }_{k}}$ of points $\left(n ; a_{1}, \ldots, a_{k}\right)$ for which $n>k$, and $n \geq a_{1} \geq a_{2} \geq \ldots \geq a_{k} \geq 1$ :

$$
\left\{\prod_{i=1}^{k}\left(I-A_{i}^{-1}\right)\right\} C_{k}\left(n ; a_{1}, \ldots, a_{k}\right)=C_{k}\left(n-1 ; a_{1}, \ldots, a_{k}\right) \cdot \quad\left(P \Delta E\left(C_{k}\right)\right)
$$

$C_{k}\left(n ; a_{1}, \ldots, a_{k}\right)$ also satisfies the following initial/boundary conditions for $\left(n ; a_{1}, \ldots, a_{k}\right) \in \overline{\text { Land_Of_Magog }_{k}}$.

For $i=1,2, \ldots, k-1$,

$$
C_{k}\left(n ; a_{1}, \ldots, a_{k}\right)=0 \quad, \quad \text { on } \quad a_{i}-a_{i+1}=-1
$$

Also:

$$
C_{k}\left(n ; a_{1}, \ldots, a_{k}\right)=0 \quad, \quad \text { on } \quad a_{k}=0
$$

Furthermore:

$$
\begin{gathered}
C_{k}\left(n ; n+1, a_{2}, \ldots, a_{k}\right)=0, \text { and } \\
C_{k}\left(k ; a_{1}, \ldots, a_{k-1}, a_{k}\right)=C_{k-1}\left(k ; a_{1}, \ldots, a_{k-1}\right) \delta\left(a_{k}, 1\right),
\end{gathered}
$$

and

$$
C_{1}\left(1 ; a_{1}\right)=\delta\left(1, a_{1}\right) \quad, \quad\left(1 ; a_{1}\right) \in \overline{{\operatorname{Land\_ } \_f_{-} M_{a g o g}}_{1}} \quad .
$$

[ Type 'S1112(k):' in ROBBINS, for specific k.]

Proof: $\left(P \Delta E\left(C_{k}\right)\right)$ follows from crucial fact $\aleph_{3}$. (In fact it holds for all $\left(n ; a_{1}, \ldots, a_{k}\right)$, without the indicated restriction, but we only need it there.)

$\left(C 1_{i}\right),(1 \leq i<k)$, is satisfied by crucial fact $\aleph_{1} .\left(C 1_{k}\right)$ is satisfied, since when $a_{k}=0$, the constant-termand of the constant term expression defining $C_{k}$ is a multiple of $x_{k}$, and hence its constant term w.r.t. to $x_{k}$, and hence w.r.t. to all variables, is $0 .(C 2)$ is satisfied thanks to crucial fact $\aleph_{2}$, since the degree of the numerator in $x_{1}, 2 k-1$ is $\leq 2(n+k-1)$ (because we have $n \geq k \geq 1$.)

The proof of $(C 3)$ is not quite so easy. First let's prove $(C 3)$ when $a_{k}=1$. Since

$$
\Delta_{k}\left(x_{1}, \ldots, x_{k}\right)=\Delta_{k-1}\left(x_{1}, \ldots, x_{k-1}\right) \cdot\left(1-2 x_{k}\right) \prod_{i=1}^{k-1}\left(x_{k}-x_{i}\right)\left(x_{k}-\bar{x}_{i}\right)
$$


we have

$$
\begin{gathered}
C_{k}\left(k ; a_{1}, \ldots, a_{k-1}, 1\right)= \\
C T_{x_{1}, \ldots, x_{k-1}}\left\{\frac{\Delta_{k-1}\left(x_{1}, \ldots, x_{k-1}\right)}{\prod_{i=1}^{k-1}\left(x_{i}^{a_{i}+k-i-1} \bar{x}_{i}^{2 k}\right)} \cdot C T_{x_{k}}\left[\frac{\left(1-2 x_{k}\right) \prod_{i=1}^{k-1}\left(x_{i}-x_{k}\right)\left(\bar{x}_{i}-x_{k}\right)}{x_{k}^{0}\left(\bar{x}_{k}\right)^{2 k}}\right]\right\} \\
=C T_{x_{1}, \ldots, x_{k-1}}\left\{\frac{\Delta_{k-1}\left(x_{1}, \ldots, x_{k-1}\right)}{\prod_{i=1}^{k-1}\left(x_{i}^{a_{i}+k-i-1} \bar{x}_{i}^{2 k}\right)} \cdot\left[\prod_{i=1}^{k-1}\left(x_{i} \bar{x}_{i}\right)\right]\right\} \\
=C T_{x_{1}, \ldots, x_{k-1}}\left\{\frac{\Delta_{k-1}\left(x_{1}, \ldots, x_{k-1}\right)}{\prod_{i=1}^{k-1}\left(x_{i}^{a_{i}+(k-1)-i-1} \bar{x}_{i}^{(k-1)+k}\right)}\right\}=C_{k-1}\left(k ; a_{1}, \ldots, a_{k-1}\right) .
\end{gathered}
$$

We will now show that $(C 3)$ holds when $a_{k} \geq 2$. When $\left(k ; a_{1}, \ldots, a_{k-1}, a_{k}\right) \in \overline{\text { Land_Of_Magog }_{k}}$ does not satisfy $k \geq a_{1} \geq a_{2} \geq \ldots \geq a_{k}$, then the left side of $(C 3)$ is already known to be 0 in virtue of $(C 1)$ or $(C 2)$. We are left with the task of showing that when $k \geq a_{1} \geq \ldots \geq a_{k} \geq 2$, the following expression vanishes:

$$
\begin{gathered}
C_{k}\left(k ; a_{1}, \ldots, a_{k}\right)=C T_{x_{1}, \ldots, x_{k}}\left\{\frac{\Delta_{k}\left(x_{1}, \ldots, x_{k}\right)}{\prod_{i=1}^{k} x_{i}^{a_{i}+k-i-1}\left(\bar{x}_{i}\right)^{2 k}}\right\}=C T_{x_{1}, \ldots, x_{k}}\left\{\frac{\left[\prod_{i=1}^{k} x_{i}^{k-a_{i}+i}\right] \Delta_{k}\left(x_{1}, \ldots, x_{k}\right)}{\prod_{i=1}^{k} x_{i}^{2 k-1}\left(\bar{x}_{i}\right)^{2 k}}\right\} \\
=\operatorname{Res}_{x_{1}, \ldots, x_{k}}\left\{\frac{\left[\prod_{i=1}^{k} x_{i}^{k-a_{i}+i}\right] \Delta_{k}\left(x_{1}, \ldots, x_{k}\right)}{\prod_{i=1}^{k} x_{i}^{2 k}\left(\bar{x}_{i}\right)^{2 k}}\right\} .
\end{gathered}
$$

In order to show that the right side of $(E f e s)$ indeed vanishes, we will first prove a $(s u b)^{4}$ lemma that asserts that, under the stated conditions on the $a_{i}$, the right side of (Efes) remains invariant whenever its residuand is replaced by any of its images under the action of the group of signed permutations $W\left(\mathcal{B}_{k}\right)$.

Subsubsubsublemma 1.1.1.2.1: Let $F_{k ; a_{1}, \ldots, a_{k}}\left(x_{1}, \ldots, x_{k}\right)$ be the residuand of the right side of (Efes), i.e.

$$
F_{k ; a_{1}, \ldots, a_{k}}\left(x_{1}, \ldots, x_{k}\right):=\frac{\left[\prod_{i=1}^{k} x_{i}^{k-a_{i}+i}\right] \Delta_{k}\left(x_{1}, \ldots, x_{k}\right)}{\prod_{i=1}^{k} x_{i}^{2 k}\left(\bar{x}_{i}\right)^{2 k}},
$$

where $k \geq a_{1} \geq a_{2} \ldots \geq a_{k} \geq 2$, and $\Delta_{k}$ is defined in Eq. (Delta) in the statement of sublemma 1.1. Then for any signed permutation $g=(\pi, \varepsilon) \in W\left(\mathcal{B}_{k}\right)$, we have

$$
\operatorname{Res}_{x_{1}, \ldots, x_{k}}\left\{g\left[F_{k ; a_{1}, \ldots, a_{k}}(x)\right]\right\}=\operatorname{Res}_{x_{1}, \ldots, x_{k}}\left\{F_{k ; a_{1}, \ldots, a_{k}}(x)\right\} \quad .
$$

[ Type 'S11121(k, a) :' in ROBBINS, for specific k and a.]

Proof: $\Delta_{k}$, defined in (Delta), is a polynomial of degree $2 k-1$ in any one of its variables. By Crucial Fact $\aleph_{5}^{\prime}$ we can repeatedly change all the -1 's in $\varepsilon$ to +1 's since the numerator is a polynomial of degree $\leq(2 k-1)+(k+k-2)=4 k-3 \leq 2(2 k-1)$. Finally we can change the $\pi$ in 
$g$ into the identity element, by Crucial Fact $\aleph_{4}^{\prime}$. This completes the proof of $(s u b)^{4}$ lemma 1.1.1.2.1.

We can now replace the right side of $(E f e s)$ by the iterated residue of the average of all its images under $W\left(\mathcal{B}_{k}\right)$ :

$$
\begin{gathered}
\operatorname{Res}_{x_{1}, \ldots, x_{k}}\left\{F_{k ; a_{1}, \ldots, a_{k}}(x)\right\}=\frac{1}{2^{k} k !} \sum_{g \in W\left(\mathcal{B}_{k}\right)} \operatorname{Res}_{x_{1}, \ldots, x_{k}}\left\{g\left[F_{k ; a_{1}, \ldots, a_{k}}(x)\right]\right\} \\
=\frac{1}{2^{k} k !} \operatorname{Res}_{x_{1}, \ldots, x_{k}}\left\{\sum_{g \in W\left(\mathcal{B}_{k}\right)} g\left[F_{k ; a_{1}, \ldots, a_{k}}(x)\right]\right\} .
\end{gathered}
$$

We now will prove that not only is the iterated residue on the right side of (Efes') identically zero, but so is the whole residuand.

Subsubsubsublemma 1.1.1.2.2: Let $F_{k ; a_{1}, \ldots, a_{k}}\left(x_{1}, \ldots, x_{k}\right)$ be the residuand of the right side of (Efes), i.e.

$$
F_{k ; a_{1}, \ldots, a_{k}}\left(x_{1}, \ldots, x_{k}\right):=\frac{\left[\prod_{i=1}^{k} x_{i}^{k-a_{i}+i}\right] \Delta_{k}\left(x_{1}, \ldots, x_{k}\right)}{\prod_{i=1}^{k} x_{i}^{2 k}\left(\bar{x}_{i}\right)^{2 k}}
$$

where $k \geq a_{1} \geq a_{2} \ldots \geq a_{k} \geq 2$, and $\Delta_{k}$ is defined in Eq. (Delta) in the statement of sublemma 1.1. Then

$$
\sum_{g \in W\left(\mathcal{B}_{k}\right)} g\left[F_{k ; a_{1}, \ldots, a_{k}}(x)\right]=0
$$

[ Type 'S11122(k,a) :' in ROBBINS, for specific $k$ and a.]

Proof: By the symmetry of the denominator and the anti-symmetry of $\Delta_{k}$ w.r.t. the group of signed permutations $W\left(\mathcal{B}_{k}\right)$, we have:

$$
\sum_{g \in W\left(\mathcal{B}_{k}\right)} g\left[F_{k ; a_{1}, \ldots, a_{k}}(x)\right]=\frac{\left\{\sum_{g \in W\left(\mathcal{B}_{k}\right)} \operatorname{sgn}(g) \cdot g\left[\prod_{i=1}^{k} x_{i}^{k-a_{i}+i}\right]\right\} \Delta_{k}\left(x_{1}, \ldots, x_{k}\right)}{\prod_{i=1}^{k} x_{i}^{2 k}\left(\bar{x}_{i}\right)^{2 k}} \cdot\left(\text { Efes }^{\prime \prime \prime}\right)
$$

By crucial fact $\aleph_{7}^{\prime}$, the expression inside the braces of $\left(E f e s^{\prime \prime \prime}\right)$ is a $W\left(\mathcal{B}_{k}\right)$ - anti-symmetric polynomial. By crucial fact $\aleph_{8}^{\prime}$, it is divisible by $\Delta_{k}\left(x_{1}, \ldots, x_{k}\right)$. The degree in $x_{1}$ of the polynomial inside the braces of $\left(E f e s^{\prime \prime \prime}\right)$ is $\leq k-2+k=2 k-2$, while the degree of $\Delta_{k}(x)$, in $x_{1}$, is $2 k-1$. Hence it must be the zero polynomial. This completes the proof of subsubsubsublemma 1.1.1.2.2

This completes the proof that $C_{k}$ satisfies $(C 3)$. Finally $(C 4)$ is completely routine. This completes the proof of subsubsublemma 1.1.1.2.

Subsubsublemma 1.1.1.3: There is a unique sequence of discrete functions $X_{k}\left(n ; a_{1}, \ldots, a_{k}\right)$, defined for $k \geq 1$ and $\left(n ; a_{1}, \ldots, a_{k}\right) \in \overline{{\operatorname{Land} \_O f_{-} M a g o g_{k}}_{1}}$, satisfying the following partial difference 
equation:

$$
\left\{\prod_{i=1}^{k}\left(I-A_{i}^{-1}\right)\right\} X_{k}\left(n ; a_{1}, \ldots, a_{k}\right)=X_{k}\left(n-1 ; a_{1}, \ldots, a_{k}\right)
$$

for $n>k$ and $n \geq a_{1} \geq a_{2} \geq \ldots \geq a_{k} \geq 1$, and the following boundary/initial conditions for $\left(n ; a_{1}, \ldots, a_{k}\right) \in \overline{\text { Land_Of_Magog }_{k}}$.

For $i=1,2, \ldots, k-1$,

$$
X_{k}\left(n ; a_{1}, \ldots, a_{k}\right)=0 \quad, \quad \text { on } \quad a_{i}-a_{i+1}=-1
$$

Also:

$$
X_{k}\left(n ; a_{1}, \ldots, a_{k}\right)=0 \quad, \quad \text { on } \quad a_{k}=0 .
$$

Furthermore:

$$
\begin{gathered}
X_{k}\left(n ; n+1, a_{2}, \ldots, a_{k}\right)=0 \text { and } \\
X_{k}\left(k ; a_{1}, \ldots, a_{k-1}, a_{k}\right)=X_{k-1}\left(k ; a_{1}, \ldots, a_{k-1}\right) \delta\left(a_{k}, 1\right)
\end{gathered}
$$

and

$$
X_{1}\left(1 ; a_{1}\right)=\delta\left(1, a_{1}\right) \quad, \quad\left(1 ; a_{1}\right) \in \overline{\text { Land_Of_Magog }_{1}} .
$$

[ Type 'S1113(k,n):' in ROBBINS, for specific $k$ and $n$.

Proof:

Subsublemma 1.1.1 now follows from subsubsublemmas 1.1.1.1, 1.1.1.2, and 1.1.1.3. This completes the proof of subsublemma 1.1.1.

Subsublemma 1.1.1 gave us a constant term expression for the number of $n \times k$ - Magog trapezoids with a prescribed rightmost border. In order to complete the proof of sublemma 1.1, we would have to sum them all up. In order to establish the constant term expression (MagogTotal) for $b_{k}(n)$, we would need to go through an intermediary, not-so-nice expression, given by subsublemma 1.1.2 below.

Subsublemma 1.1.2: The number of $n \times k$ Magog-trapezoids, $b_{k}(n)$, is given by the following constant term expression:

$b_{k}(n)=C T_{x_{1}, \ldots, x_{k}}\left\{\frac{x_{1} x_{2}^{2} \ldots x_{k}^{k} \cdot \Delta_{k}\left(x_{1}, \ldots, x_{k}\right)}{\prod_{i=1}^{k}\left(\bar{x}_{i}\right)^{k+n} x_{i}^{n+k-1}} \cdot \frac{1}{\left(1-x_{k}\right)\left(1-x_{k-1} x_{k}\right) \ldots\left(1-x_{k} x_{k-1} \ldots x_{1}\right)}\right\}$

(George)

[ Type 'S112(k,n):' in ROBBINS, for specific $\mathrm{k}$ and $\mathrm{n}$.]

Proof: We will establish (George) by starting from the right side, and proving that it equals the left side. By the well-known symmetry property of the '=' relation, it would follow that the left side is equal to its right side. 
Since $(1-y)^{-1}=\sum_{\alpha=0}^{\infty} y^{\alpha}$, we have

$$
\begin{gathered}
\left(1-x_{k}\right)^{-1}\left(1-x_{k-1} x_{k}\right)^{-1} \ldots\left(1-x_{k} x_{k-1} \ldots x_{1}\right)^{-1}=\left[\sum_{\alpha_{k}=0}^{\infty} x_{k}^{\alpha_{k}}\right]\left[\sum_{\alpha_{k-1}=0}^{\infty}\left(x_{k-1} x_{k}\right)^{\alpha_{k-1}}\right] \ldots\left[\sum_{\alpha_{1}=0}^{\infty}\left(x_{1} \ldots x_{k-1} x_{k}\right)^{\alpha_{1}}\right] \\
=\sum_{0 \leq \alpha_{1}, \ldots, \alpha_{k}<\infty} x_{1}^{\alpha_{1}} x_{2}^{\alpha_{1}+\alpha_{2}} \ldots x_{k}^{\alpha_{1}+\alpha_{2}+\ldots+\alpha_{k}}=\sum_{0 \leq \lambda_{1} \leq \ldots \leq \lambda_{k}<\infty} x_{1}^{\lambda_{1}} \ldots x_{k}^{\lambda_{k}}
\end{gathered}
$$

The right side of (George) is hence equal to:

$$
\begin{gathered}
C T_{x_{1}, \ldots, x_{k}}\left\{\frac{x_{1} x_{2}^{2} \ldots x_{k}^{k} \cdot \Delta_{k}\left(x_{1}, \ldots, x_{k}\right)}{\prod_{i=1}^{k}\left(\bar{x}_{i}\right)^{k+n} x_{i}^{n+k-1}} \cdot \sum_{0 \leq \lambda_{1} \leq \ldots \leq \lambda_{k}<\infty} x_{1}^{\lambda_{1}} \ldots x_{k}^{\lambda_{k}}\right\} \\
=\sum_{0 \leq \lambda_{1} \leq \ldots \leq \lambda_{k}<\infty} C T_{x_{1}, \ldots, x_{k}}\left\{\frac{x_{1} x_{2}^{2} \ldots x_{k}^{k} \cdot \Delta_{k}\left(x_{1}, \ldots, x_{k}\right)}{\prod_{i=1}^{k}\left(\bar{x}_{i}\right)^{k+n} x_{i}^{\left(n-\lambda_{i}\right)+k-1}}\right\} \\
=\sum_{n \geq\left(n-\lambda_{1}\right) \geq \ldots \geq\left(n-\lambda_{k}\right)>-\infty} C T_{x_{1}, \ldots, x_{k}}\left\{\frac{x_{1} x_{2}^{2} \ldots x_{k}^{k} \cdot \Delta_{k}\left(x_{1}, \ldots, x_{k}\right)}{\prod_{i=1}^{k}\left(\bar{x}_{i}\right)^{k+n} x_{i}^{\left(n-\lambda_{i}\right)+k-1}}\right\} \\
=\sum_{n \geq a_{1} \geq \ldots \geq a_{k}>-\infty} C T_{x_{1}, \ldots, x_{k}}\left\{\frac{x_{1} x_{2}^{2} \ldots x_{k}^{k} \cdot \Delta_{k}\left(x_{1}, \ldots, x_{k}\right)}{\prod_{i=1}^{k}\left(\bar{x}_{i}\right)^{k+n} x_{i}^{a_{i}+k-1}}\right\} .
\end{gathered}
$$

The sum on the right side of $($ George $)$ can be replaced by a sum over the restricted region $n \geq$ $a_{1} \geq \ldots \geq a_{k} \geq 1$, since whenever $a_{k}<1$, the constant-termand there is a multiple of $x_{k}$, and hence its constant term is 0 . So the right side of (George') (and hence the right side of (George)) equals:

$$
\begin{gathered}
\sum_{n \geq a_{1} \geq \ldots \geq a_{k} \geq 1} C T_{x_{1}, \ldots, x_{k}}\left\{\frac{x_{1} x_{2}^{2} \ldots x_{k}^{k} \cdot \Delta_{k}\left(x_{1}, \ldots, x_{k}\right)}{\prod_{i=1}^{k}\left(\bar{x}_{i}\right)^{k+n} x_{i}^{a_{i}+k-1}}\right\} \\
=\sum_{n \geq a_{1} \geq \ldots \geq a_{k} \geq 1} C_{k}\left(n ; a_{1}, \ldots, a_{k}\right)=\sum_{n \geq a_{1} \geq \ldots \geq a_{k} \geq 1} B_{k}\left(n ; a_{1}, \ldots, a_{k}\right)=b_{k}(n) \quad, \quad\left(\text { George }^{\prime \prime}\right)
\end{gathered}
$$

where the last equality in (George $\left.{ }^{\prime \prime}\right)$ is obvious, and the second-to-last equality follows from subsublemma 1.1.1. This completes the proof of subsublemma 1.1.2.

By crucial fact $\aleph_{4}$, we can replace the right side of (George) by the constant term of the average of all the $\mathcal{S}_{k}$-images of its constant-termand. Since $\Delta_{k}$ is antisymmetric in $\left(x_{1}, \ldots, x_{k}\right)$, we see that $b_{k}(n)$ equals

$$
\frac{1}{k !} C T_{x_{1}, \ldots, x_{k}}\left\{\frac{\Delta_{k}\left(x_{1}, \ldots, x_{k}\right)}{\prod_{i=1}^{k}\left(\bar{x}_{i}\right)^{k+n} x_{i}^{n+k-1}}\left(\sum_{\pi \in \mathcal{S}_{k}} \operatorname{sgn}(\pi) \cdot \pi\left[\frac{x_{1} x_{2}^{2} \ldots x_{k}^{k}}{\left(1-x_{k}\right)\left(1-x_{k} x_{k-1}\right) \ldots\left(1-x_{k} x_{k-1} \ldots x_{1}\right)}\right]\right)\right\} .
$$

(Stanley)

We now need: 


\section{Subsublemma 1.1.3:}

$$
\sum_{\pi \in \mathcal{S}_{k}} \operatorname{sgn}(\pi) \cdot \pi\left[\frac{x_{1} x_{2}^{2} \ldots x_{k}^{k}}{\left(1-x_{k}\right)\left(1-x_{k} x_{k-1}\right) \cdots\left(1-x_{k} x_{k-1} \ldots x_{1}\right)}\right]=\frac{x_{1} \cdots x_{k} \prod_{1 \leq i<j \leq k}\left(x_{j}-x_{i}\right)}{\prod_{i=1}^{k}\left(1-x_{i}\right) \prod_{1 \leq i<j \leq k}\left(1-x_{i} x_{j}\right)} .
$$

[ Type 'S113(k);' in ROBBINS, for specific k.]

Proof : See [PS], problem VII.47. Alternatively, (Issai) is easily seen to be equivalent to Schur's identity that sums all the Schur functions ([Ma], ex I.5.4, p. 45). This takes care of subsublemma 1.1.3.

Inserting (Issai) into (Stanley), expanding $\prod_{1 \leq i<j \leq k}\left(x_{j}-x_{i}\right)$ by Vandermonde's expansion,

$$
\sum_{\pi \in \mathcal{S}_{k}} \operatorname{sgn}(\pi) \cdot \pi\left(x_{1}^{0} x_{2}^{1} \cdots x_{k}^{k-1}\right)
$$

using the antisymmetry of $\Delta_{k}$ once again, and employing crucial fact $\aleph_{4}$, we get the following string of equalities:

$$
\begin{gathered}
b_{k}(n)=\frac{1}{k !} C T_{x_{1}, \ldots, x_{k}}\left\{\frac{\Delta_{k}\left(x_{1}, \ldots, x_{k}\right)}{\prod_{i=1}^{k}\left(\bar{x}_{i}\right)^{k+n} x_{i}^{n+k-1}}\left(\frac{x_{1} \cdots x_{k} \prod_{1 \leq i<j \leq k}\left(x_{j}-x_{i}\right)}{\prod_{i=1}^{k}\left(1-x_{i}\right) \prod_{1 \leq i<j \leq k}\left(1-x_{i} x_{j}\right)}\right)\right\} \\
=\frac{1}{k !} C T_{x_{1}, \ldots, x_{k}}\left\{\frac{\Delta_{k}\left(x_{1}, \ldots, x_{k}\right)}{\prod_{i=1}^{k}\left(\bar{x}_{i}\right)^{k+n+1} x_{i}^{n+k-2} \prod_{1 \leq i<j \leq k}\left(1-x_{i} x_{j}\right)}\left(\sum_{\pi \in \mathcal{S}_{k}} \operatorname{sgn}(\pi) \cdot \pi\left(x_{1}^{0} x_{2}^{1} \ldots x_{k}^{k-1}\right)\right)\right\} \\
=\frac{1}{k !} \sum_{\pi \in \mathcal{S}_{k}} C T_{x_{1}, \ldots, x_{k}}\left\{\pi\left[\frac{\Delta_{k}\left(x_{1}, \ldots, x_{k}\right)}{\prod_{i=1}^{k}\left(\bar{x}_{i}\right)^{k+n+1} x_{i}^{n+k-2} \prod_{1 \leq i<j \leq k}\left(1-x_{i} x_{j}\right)}\left(\prod_{i=1}^{k} x_{i}^{i-1}\right)\right]\right\} \\
=\frac{1}{k !} \sum_{\pi \in \mathcal{S}_{k}} C T_{x_{1}, \ldots, x_{k}}\left\{\pi\left[\frac{\Delta_{k}\left(x_{1}, \ldots, x_{k}\right)}{\prod_{i=1}^{k}\left(\bar{x}_{i}\right)^{k+n+1} x_{i}^{n+k-i-1} \prod_{1 \leq i<j \leq k}\left(1-x_{i} x_{j}\right)}\right]\right\} \\
=\frac{1}{k !} \sum_{\pi \in \mathcal{S}_{k}} C T_{x_{1}, \ldots, x_{k}}\left\{\frac{\Delta_{k}\left(x_{1}, \ldots, x_{k}\right)}{\prod_{i=1}^{k}\left(\bar{x}_{i}\right)^{k+n+1} x_{i}^{n+k-i-1} \prod_{1 \leq i<j \leq k}\left(1-x_{i} x_{j}\right)}\right\} \\
=\frac{1}{k !}\left(\sum_{\pi \in \mathcal{S}_{k}} 1\right) C T_{x_{1}, \ldots, x_{k}}\left\{\frac{\Delta_{k}\left(x_{1}, \ldots, x_{k}\right)}{\prod_{i=1}^{k}\left(\bar{x}_{i}\right)^{k+n+1} x_{i}^{n+k-i-1} \prod_{1 \leq i<j \leq k}\left(1-x_{i} x_{j}\right)}\right\} \\
=C T_{x_{1}, \ldots, x_{k}}\left\{\frac{\Delta_{k}\left(x_{1}, \ldots, x_{k}\right)}{\prod_{i=1}^{k}\left(\bar{x}_{i}\right)^{k+n+1} x_{i}^{n+k-i-1} \prod_{1 \leq i<j \leq k}\left(1-x_{i} x_{j}\right)}\right\},
\end{gathered}
$$

where in the last equality we have used Levi Ben Gerson's celebrated result that the number of elements in $\mathcal{S}_{k}$ (the symmetric group on $k$ elements,) equals $k$ !. The extreme right of $\left(\right.$ George $\left.e^{\prime \prime \prime}\right)$ is exactly the right side of (MagogTotal). This completes the proof of sublemma 1.1. 


\section{Act II. COUNTING GOG}

And say, Thus saith the Lord God; Behold, I am against thee, O Gog...

(Ezekiel XXXIIX,3)

An $n \times k-$ Gog trapezoid (where $n \geq k \geq 1$ ) is a trapezoidal array of integers

$$
\left(d_{i, j}\right) \quad, \quad 1 \leq i \leq n \quad, \quad 1 \leq j \leq \min (k, n+1-i)
$$

such that:

$$
\begin{gathered}
\text { (i) } \quad d_{i, j}<d_{i, j+1} \quad(i i) \quad d_{i, j} \leq d_{i+1, j} \quad(i i i) \quad d_{i, j} \geq d_{i+1, j-1} \\
(i v) \quad d_{1, j}=j \quad \text { (v) } \quad d_{i, k} \leq i+k-1 .
\end{gathered}
$$

(Note that these five conditions imply that $1 \leq d_{i, j} \leq n$.)

[ To view all $\mathrm{n}$ by $\mathrm{k}$ Gog trapezoids, for any given $\mathrm{k}$ and $\mathrm{n}$, type 'GOG(k,n):' in ROBBINS. For example, GOG(3,4) would display all 4 by 3 Gog trapezoids.]

Sublemma 1.2: The total number of $n \times k-$ Gog trapezoids, let's call it $m_{k}(n)$, is given by the following constant term expression:

$$
m_{k}(n)=C T_{x_{1}, \ldots, x_{k}}\left[\frac{\Phi_{k}\left(x_{1}, \ldots, x_{k}\right)}{\left\{\prod_{i=1}^{k} x_{i}^{n}\left(\bar{x}_{i}\right)^{n+i+1}\right\}\left\{\prod_{1 \leq i<j \leq k}\left(1-x_{i} x_{j}\right)\left(1-\bar{x}_{i} x_{j}\right)\right\}}\right] \quad, \quad \text { (GogTotal) }
$$

where the polynomial $\Phi_{k}\left(x_{1}, \ldots, x_{k}\right)$ is defined by:

$$
\Phi_{k}\left(x_{1}, \ldots, x_{k}\right)=(-1)^{k} \sum_{g \in W\left(\mathcal{B}_{k}\right)} \operatorname{sgn}(g) \cdot g\left[\prod_{i=1}^{k} \bar{x}_{i}^{k-i} x_{i}^{k} \prod_{1 \leq i<j \leq k}\left(1-x_{i} \bar{x}_{j}\right)\left(1-\bar{x}_{i} \bar{x}_{j}\right)\right]
$$

[ Type 'S12(k,n):' in ROBBINS, for specific $k$ and $n$.]

Proof: Let $M_{k}\left(n ; a_{1}, a_{2}, \ldots, a_{k}\right)$ be the number of $n \times k$ - Gog trapezoids

$$
\left(d_{i, j}\right), \quad 1 \leq i \leq n \quad, \quad 1 \leq j \leq \min (k, n+1-i) \quad,
$$

such that $d_{n-k+i, k-i+1}=a_{i}$, for $i=1, \ldots, k$. In other words:

$$
d_{n-k+1, k}=a_{1} \quad, \quad d_{n-k+2, k-1}=a_{2} \quad, \quad \ldots \quad, \quad d_{n-k+i, k-i+1}=a_{i} \quad, \quad \ldots \quad, \quad d_{n, 1}=a_{k} \quad .
$$

The conceivable $\left(n ; a_{1}, \ldots, a_{k}\right)$ range over the set

$$
\begin{gathered}
\operatorname{Land\_ Of\_ Gog}_{k}:=\left\{\left(n ; a_{1}, \ldots, a_{k}\right) \mid\right. \\
\left.n \geq k, \quad n \geq a_{1} \geq a_{2} \geq \ldots \geq a_{k} \geq 1 \quad, \quad a_{1} \geq k \quad, \quad a_{2} \geq k-1 \quad, \quad \ldots \quad, \quad a_{k} \geq 1\right\} .
\end{gathered}
$$


[ To view this set, for specific $k$ and $n$, type 'LOGOG $(k, n)$ :' in ROBBINS.]

For any $\left(n ; a_{1}, \ldots, a_{k}\right) \in L_{a n d \_O f_{-} G o g_{k}}$, define the set:

$\widetilde{T}_{k}\left(n ; a_{1}, \ldots, a_{k}\right):=\left\{\left(b_{1}, \ldots, b_{k}\right) \quad \mid \quad n-1 \geq b_{1} \geq b_{2} \geq \ldots \geq b_{k} \geq 1 \quad, \quad k-i+1 \leq b_{i} \leq a_{i}\right\} \quad$.

It turns out to be convenient (and most likely necessary) to introduce a related discrete function

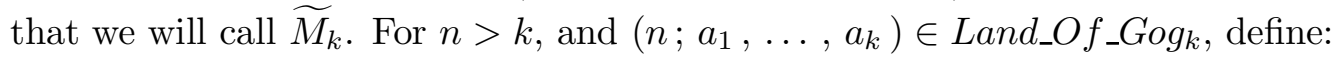

$$
\widetilde{M}_{k}\left(n ; a_{1}, a_{2}, \ldots, a_{k}\right):=\sum_{b \in \widetilde{T}_{k}\left(n ; a_{1}, \ldots, a_{k}\right)} M_{k}\left(n-1 ; b_{1}, \ldots, b_{k}\right)
$$

and when $n=k>1$ define $\widetilde{M}_{k}(k ; k,-, \ldots,-)$ by

$$
\widetilde{M}_{k}\left(k ; k, a_{2}, \ldots, a_{k}\right)=\widetilde{M}_{k-1}\left(k ; a_{2}, \ldots, a_{k}\right) \quad,(k>1) \quad, \quad\left(\widetilde{M}_{k}(n=k)\right)
$$

while for $n=k=1$ define

$$
\widetilde{M}_{1}(1 ; 1):=1 \quad\left(\widetilde{M}_{k}(n=k=1)\right)
$$

The following subsublemma gives a constant term expression for $\widetilde{M}_{k}$.

Subsublemma 1.2.1: Let

$$
H_{k}\left(n ; a_{1}, \ldots, a_{k}\right):=C T_{x_{1}, \ldots, x_{k}}\left\{\frac{\Phi_{k}\left(x_{1}, \ldots, x_{k}\right)}{\left\{\prod_{i=1}^{k} x_{i}^{a_{i}-1}\left(\bar{x}_{i}\right)^{n+i}\right\} \prod_{1 \leq i<j \leq k}\left(1-x_{i} x_{j}\right)\left(1-\bar{x}_{i} x_{j}\right)}\right\}
$$

where $\Phi_{k}$ is defined in the statement of sublemma 1.2 above. For all $\left(n ; a_{1}, \ldots, a_{k}\right) \in L_{a n d \_O f \_G o g}$, we have

$$
\widetilde{M}_{k}\left(n ; a_{1}, \ldots, a_{k}\right)=H_{k}\left(n ; a_{1}, \ldots, a_{k}\right)
$$

[ Type 'S121 $(\mathrm{k}, \mathrm{n})$ :' in ROBBINS, for specific $\mathrm{k}$ and $\mathrm{n}$.

Proof: It is convenient to extend $\widetilde{M}_{k}$ to the following larger domain (except that we slightly 'chop' it when $n=k)$ :

$$
\begin{gathered}
\overline{\text { Land_Of_Gog }_{k}}:=\left\{\quad\left(n ; a_{1}, \ldots, a_{k}\right) \quad \mid n \geq k, n-a_{1} \geq-1,\right. \\
a_{1} \geq a_{2} \geq \ldots \geq a_{k} \quad, \quad a_{1} \geq k-1 \quad, \quad a_{2} \geq k-2 \quad, \quad \ldots \quad, \quad a_{k} \geq 0 \\
\text { and } \left.\left.a_{1}=n+1 \Rightarrow a_{2} \leq n \quad \text { and } \quad n=k=a_{1} \Rightarrow a_{2}<k\right)\right\} \quad,
\end{gathered}
$$

[ To view this set, for specific $\mathrm{k}$ and $\mathrm{n}$, type 'ELOGOG (k,n):' in ROBBINS], and to define $\widetilde{M}_{k}$ to be 0 at all the

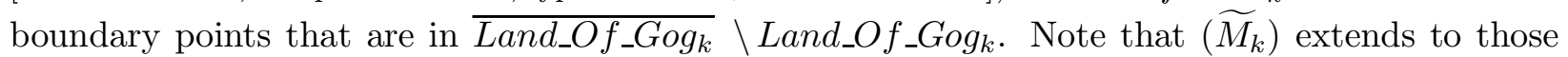
boundary points for which $a_{i}=k-i$ for one or more $i$ 's, since then the summation-set, $\widetilde{T}_{k}$, is the empty set. However, $\left(\widetilde{M}_{k}\right)$ does not hold for those boundary points for which $a_{1}=n+1$. 
We will prove that subsublemma 1.2.1 even holds in this larger domain $\overline{\text { and_Of_Gog }_{k}}$. This would follow from the following three subsubsublemmas.

Subsubsublemma 1.2.1.1: Let $k \geq 1$ and for any non-increasing vector of non-negative integers $a=\left(a_{1}, \ldots, a_{k}\right)$, define the partial difference operator $P^{(a)}$ by:

$$
P^{(a)}\left(A_{1}, \ldots, A_{k}\right):=\prod_{\left\{i \mid a_{i}-a_{i+1}>0\right\}}\left(I-A_{i}^{-1}\right) \quad,
$$

where we declare that $a_{k+1}=0 . \widetilde{M}_{k}$ satisfies the following partial recurrence:

$$
\begin{gathered}
P^{(a)}\left(A_{1}, \ldots, A_{k}\right) \widetilde{M}_{k}\left(n ; a_{1}, \ldots, a_{k}\right) \\
=\widetilde{M}_{k}\left(n-1 ; a_{1}, \min \left(a_{1}-1, a_{2}\right), \ldots, \min \left(a_{k-1}-1, a_{k}\right)\right)
\end{gathered}
$$

valid whenever $n>k$ and $\left(n ; a_{1}, \ldots, a_{k}\right) \in$ Land_Of_Gog $_{k} . \widetilde{M}_{k}\left(n ; a_{1}, \ldots, a_{k}\right)$ also satisfies the following initial/boundary conditions for $\left(n ; a_{1}, \ldots, a_{k}\right) \in \overline{\text { Land_Of_Gog }_{k}}$ :

$$
\begin{gathered}
\widetilde{M}_{k}\left(n ; a_{1}, \ldots, a_{k}\right)=0 \quad \text { on } \quad a_{i}=k-i, \\
\widetilde{M}_{k}\left(n ; n+1, a_{2}, \ldots, a_{k}\right)=0, \\
\widetilde{M}_{k}\left(k ; k, a_{2}, \ldots, a_{k}\right)=\widetilde{M}_{k-1}\left(k ; a_{2}, \ldots, a_{k}\right)
\end{gathered}
$$

and, finally the "Adam" condition:

$$
\widetilde{M}_{1}(1 ; 1)=1
$$

[ Type 'S1211all $(k, n)$ :' in ROBBINS, for specific $k$ and $n$.

Proof: Consider a typical $n \times k$ Gog trapezoid $\left(d_{i, j}\right)$ that is counted by $M_{k}\left(n ; a_{1}, \ldots, a_{k}\right)$, where $n>k$ and $\left(n ; a_{1}, \ldots, a_{k}\right) \in$ Land_Of_Gog $_{k}$. This means that

$$
d_{n-k+1, k}=a_{1} \quad, \quad d_{n-k+2, k-1}=a_{2} \quad, \quad \ldots \quad, \quad d_{n-k+i, k-i+1}=a_{i} \quad, \quad \ldots \quad, \quad d_{n, 1}=a_{k} .
$$

Now let

$d_{n-k, k}=b_{1} \quad, \quad d_{n-k+1, k-1}=b_{2} \quad, \quad \ldots \quad, \quad d_{n-k+i-1, k-i+1}=b_{i} \quad, \quad \ldots \quad, \quad d_{n-1,1}=b_{k} \quad$.

By the conditions $(i)-(v)$ defining Gogs, given right at the beginning of this section, it follows that the allowed $b=\left(b_{1}, \ldots, b_{k}\right)$ range over the set

$$
\begin{gathered}
T_{k}\left(n ; a_{1}, \ldots, a_{k}\right):= \\
\left\{\left(b_{1}, \ldots, b_{k}\right) \quad \mid \quad n-1 \geq b_{1} \geq b_{2} \geq \ldots \geq b_{k} \geq 1 \quad \text { and } \quad k-i+1 \leq b_{i} \leq \min \left(a_{i}, a_{i-1}-1\right) \quad(i=1, \ldots, k)\right\} \quad,
\end{gathered}
$$

where $a_{0}:=\infty$. By deleting $d_{n, 1}=a_{k}, \quad d_{n-1,2}=a_{k-1} \quad, \quad \ldots \quad, \quad d_{n-k+1, k}=a_{1}$, we obtain a certain $(n-1) \times k-$ Gog trapezoid. Looking at all the conceivable possibilities for $b=\left(b_{1}, \ldots, b_{k}\right)$, we get the following (non-local) recurrence, valid for $n>k$, and $\left(n ; a_{1}, \ldots, a_{k}\right) \in$ Land_Of_Gog $_{k}$,

$$
M_{k}\left(n ; a_{1}, \ldots, a_{k}\right)=\sum_{b \in T_{k}\left(n ; a_{1}, \ldots, a_{k}\right)} M_{k}\left(n-1 ; b_{1}, \ldots, b_{k}\right)
$$

(Howard) 
(Howard) Can be used to compile a table of $M_{k}$ together with the obvious initial condition

$$
M_{k}\left(k ; k, a_{2}, \ldots, a_{k}\right)=M_{k-1}\left(k ; a_{2}, \ldots, a_{k}\right) \quad,(k>1),
$$

$\left(\right.$ Howard $\left.^{\prime}\right)$

(note that, when $n=k, a_{1}$ must be $k$,) and the "Adam" condition $M_{1}(1 ; 1)=1$.

Using the definition $\left(\widetilde{M}_{k}\right)$, Eq. (Howard) can be rewritten as:

$$
M_{k}\left(n ; a_{1}, \ldots, a_{k}\right)=\widetilde{M}_{k}\left(n ; a_{1}, \min \left(a_{1}-1, a_{2}\right), \ldots, \min \left(a_{k-1}-1, a_{k}\right)\right)
$$

valid for $n>k$ and $\left(n ; a_{1}, \ldots, a_{k}\right) \in L_{\text {Land_Of_Gog }}$. We are now ready to prove that $\widetilde{M}_{k}$ satisfies $\left(P \Delta E\left(\widetilde{M}_{k}\right)\right)$ in the statement of the current subsubsublemma 1.2.1.1. Note that since $a_{k}>0$, the factor $\left(I-A_{k}^{-1}\right)$ is always present in $P^{(a)}$.

Let $\left(a_{1}, \ldots, a_{k}\right)$ be arranged in maximal blocks of equal components as follows:

$$
a_{1}=a_{2}=\ldots=a_{r_{1}}>a_{r_{1}+1}=\ldots=a_{r_{2}}>\ldots>a_{r_{l-1}+1}=\ldots=a_{r_{l}} \geq 1
$$

where $r_{l}=k$, and $r_{1}, r_{2}-r_{1}, \ldots, r_{l}-r_{l-1}$, are the lengths of (maximal) blocks of consecutive equal components of $a$. Let's put

$$
c_{1}:=a_{r_{1}}, \ldots, c_{l-1}:=a_{r_{l-1}}, c_{l}:=a_{r_{l}}\left(=a_{k}\right)
$$

We have:

$$
P^{(a)}=\left(I-A_{r_{1}}^{-1}\right) \ldots\left(I-A_{r_{l}}^{-1}\right)
$$

The recurrence that we have to show, $\left(P \Delta E\left(\widetilde{M}_{k}\right)\right)$, spells out to:

$$
\begin{aligned}
& \left(I-A_{r_{1}}^{-1}\right)\left(I-A_{r_{2}}^{-1}\right) \ldots\left(I-A_{r_{l}}^{-1}\right) \widetilde{M}_{k}\left(n ; c_{1}, \ldots, c_{1}, c_{2}, \ldots, c_{2}, \ldots, \ldots, c_{l}, \ldots, c_{l}\right) \\
= & \widetilde{M}_{k}\left(n-1 ; c_{1}, c_{1}-1, \ldots, c_{1}-1, c_{2}, c_{2}-1, \ldots, c_{2}-1, \ldots, \ldots, c_{l}, c_{l}-1, \ldots, c_{l}-1\right),
\end{aligned}
$$

where the length of the ' $c_{i}$ ' block is $r_{i}-r_{i-1}$, for $i=1 \ldots l$ (where we agree that $r_{0}=0$.)

Suppose first that $c_{1}=a_{1}<n$, so that $\left(n-1 ; a_{1}, \ldots, a_{k}\right) \in$ Land_Of_Gog $_{k}$.

Applying $\left(I-A_{k}^{-1}\right)$ (note that $\left.k=r_{l}\right)$ to the sum $\left(\widetilde{M}_{k}\right)$, yields a sum over the subset:

$$
\begin{gathered}
\widetilde{T}_{k}\left(n ; a_{1}, \ldots, a_{k-1}, a_{k}\right) \backslash \widetilde{T}_{k}\left(n ; a_{1}, \ldots, a_{k-1}, a_{k}-1\right)= \\
\widetilde{T}_{k}\left(n ; c_{1}, \ldots, c_{1}, c_{2}, \ldots, c_{2}, \ldots, \ldots, c_{l-1}, \ldots, c_{l-1}, c_{l}, \ldots, c_{l}, c_{l}\right) \backslash \\
\widetilde{T}_{k}\left(n ; c_{1}, \ldots, c_{1}, c_{2}, \ldots, c_{2}, \ldots, \ldots, c_{l-1}, \ldots, c_{l-1}, c_{l}, \ldots, c_{l}, c_{l}-1\right) \\
=\left\{\left(b_{1}, \ldots, b_{k}\right) \quad \mid \quad n-1 \geq b_{1} \geq b_{2} \geq \ldots \geq b_{k} \geq 1\right. \\
\left.k-i+1 \leq b_{i} \leq a_{i} \text { for } i=1, \ldots, r_{l-1}, \text { and } b_{i}=c_{l}, \text { for } i=r_{l-1}+1, \ldots, k\right\}
\end{gathered}
$$

The last equality follows from the fact that

$$
b_{r_{l-1}+1} \geq \ldots \geq b_{r_{l}}=c_{l} \quad \text { and } \quad b_{r_{l-1}+1} \leq c_{l}
$$


implies that $b_{r_{l-1}+1}=\ldots=b_{r_{l}}=c_{l}$. Applying next $\left(I-A_{r_{l-1}}^{-1}\right)$ to this smaller sum, yields a sum with the same summand, but over the smaller subset:

$$
\begin{gathered}
\left\{\left(b_{1}, \ldots, b_{k}\right) \quad \mid \quad n-1 \geq b_{1} \geq b_{2} \geq \ldots \geq b_{k} \geq 1 \quad, \quad k-i+1 \leq b_{i} \leq a_{i}, \quad \text { for } \quad i=1, \ldots, r_{l-2}\right. \\
\left.b_{i}=c_{l-1}, \text { for } \quad i=r_{l-2}+1 \ldots r_{l-1}, \text { and } \quad b_{i}=c_{l}, \text { for } \quad i=r_{l-1}+1, \ldots, k\right\}
\end{gathered}
$$

Continuing to apply $\left(I-A_{r_{i}}^{-1}\right)$ with $i=l-2, l-3, \ldots, 1$, we keep getting a sum over continuously shrinking subsets of $\widetilde{T}_{k}$, until at the end we get the sum of $M_{k}\left(n-1 ; b_{1}, \ldots, b_{k}\right)$ over the singleton set

$$
\left\{\left(n-1 ; b_{1}, \ldots, b_{k}\right)=\left(n-1 ; a_{1}, \ldots, a_{k}\right)\right\}
$$

We have thus shown that, whenever $n>k$ and $\left(n ; a_{1}, \ldots, a_{k}\right) \in L_{a n d \_O f \_G o g}$, we have

$$
P^{(a)}\left(A_{1}, \ldots, A_{k}\right) \widetilde{M}_{k}\left(n ; a_{1}, \ldots, a_{k}\right)=M_{k}\left(n-1 ; a_{1}, \ldots, a_{k}\right) \quad .
$$

Now use (Bill), with $n$ replaced by $n-1$. ((Bill) holds with $n$ replaced by $n-1$ if $n-1>k$ and $\left(n-1 ; a_{1}, \ldots, a_{k}\right) \in$ Land_Of_Gog $_{k}$. For this we need the assumed hypothesis $\left.a_{1}<n.\right)$

This leaves those elements $\left(n ; a_{1}, \ldots, a_{k}\right)$ in Land_Of_Gog $L_{k}, n>k$, for which $\left(n-1 ; a_{1}, \ldots, a_{k}\right)$ does not lie in $L_{a n d} O f_{-} G o g_{k}$, namely the cases $n-1=k$ or $a_{1}=n$. These are done in the next two paragraphs.

When $n=k+1$, the sole surviving term, after applying $P^{(a)}$ to $\left(\widetilde{M}_{k}\right)$, is $M_{k}\left(k ; k, a_{2}, \ldots, a_{k}\right)$, which by $($ Howard $)$, equals $M_{k-1}\left(k ; a_{2}, \ldots, a_{k}\right)$, which by (Bill) equals $\widetilde{M}_{k-1}\left(k ; a_{2}, \min \left(a_{2}-\right.\right.$ $\left.\left.1, a_{3}\right), \ldots, \min \left(a_{k-1}-1, a_{k}\right)\right)$, which, in turn, by the definition $\left(\widetilde{M}_{k}(n=k)\right)$ equals $\widetilde{M}_{k}\left(k ; k, a_{2}, \min \left(a_{2}-\right.\right.$ $\left.\left.1, a_{3}\right), \ldots, \min \left(a_{k-1}-1, a_{k}\right)\right)$, which disposes of the case $n=k+1$.

Now suppose that $a_{1}=n$, so that $a_{1}=\ldots=a_{r_{1}}=n$. As before, applying $\left(I-A_{r_{2}}^{-1}\right) \ldots\left(I-A_{r_{l}}^{-1}\right)$ to the sum on the right of Eq. $\left(\widetilde{M}_{k}\right)$, leaves us a much reduced sum over the subset of $\widetilde{T}_{k}\left(n ; a_{1}, \ldots, a_{k}\right)$ for which $b_{r_{1}+1}=a_{r_{1}+1}, \ldots, b_{k}=a_{k}$. Since we must have $b_{1} \leq n-1$ this is the same set as the one for which $a_{1}=\ldots=a_{r_{1}-1}=n$ and $a_{r_{1}}=n-1$, so that applying $\left(1-A_{r_{1}}^{-1}\right)$ to that sum gives 0 . But the right side of $\left(P \Delta E\left(\widetilde{M}_{k}\right)\right)$ is also 0 then, by the extended definition of $\widetilde{M}_{k}$, since $a_{1}=n={ }^{\prime} n-1$ ' +1 , and $n=' n-1$ '.

$\left(M 1_{i}\right)$ and $(M 2)$ hold because $\widetilde{M}_{k}$ was defined to be 0 there, while $(M 3)$ is the definition at $n=k$, given by $\left(\widetilde{M}_{k}(n=k)\right)$, and $(M 4)$ follows from the definition $\left(\widetilde{M}_{1}\right)$. This completes the proof of $(s u b)^{3}$ lemma 1.2.1.1.

Subsubsublemma 1.2.1.2: Let $k \geq 1$, and for any non-increasing vector of non-negative integers $a=\left(a_{1}, \ldots, a_{k}\right)$, define the partial difference operator $P^{(a)}$ by:

$$
P^{(a)}\left(A_{1}, \ldots, A_{k}\right):=\prod_{\left\{i \mid a_{i}-a_{i+1}>0\right\}}\left(I-A_{i}^{-1}\right)
$$


where we declare that $a_{k+1}=0$. The sequence of discrete functions $H_{k}\left(n ; a_{1}, \ldots, a_{k}\right),(n \geq k \geq 1)$, defined in the statement of the subsublemma 1.2.1, satisfies the following partial recurrence:

$$
\begin{gathered}
P^{(a)}\left(A_{1}, \ldots, A_{k}\right) H_{k}\left(n ; a_{1}, \ldots, a_{k}\right) \\
=H_{k}\left(n-1 ; a_{1}, \min \left(a_{1}-1, a_{2}\right), \ldots, \min \left(a_{k-1}-1, a_{k}\right)\right) \quad, \quad\left(P \Delta E\left(H_{k}\right)\right)
\end{gathered}
$$

valid whenever $n>k$ and $\left(n ; a_{1}, \ldots, a_{k}\right) \in{\operatorname{Land} \_O f_{-} G_{o g} .}_{k} H_{k}\left(n ; a_{1}, \ldots, a_{k}\right)$ also satisfies the following initial/boundary conditions for $\left(n ; a_{1}, \ldots, a_{k}\right) \in \overline{\text { Land_Of_Gog }_{k}}$ :

$$
\begin{gathered}
H_{k}\left(n ; a_{1}, \ldots, a_{k}\right)=0 \quad \text { on } \quad a_{i}=k-i \\
H_{k}\left(n ; n+1, a_{2}, \ldots, a_{k}\right)=0 \\
H_{k}\left(k ; k, a_{2}, \ldots, a_{k}\right)=H_{k-1}\left(k ; a_{2}, \ldots, a_{k}\right)
\end{gathered}
$$

and, finally the "Adam" condition

$$
H_{1}(1 ; 1)=1
$$

Proof: The task of proving this $(s u b)^{3}$ lemma will be divided between the following $(s u b)^{4}$ lemmas: 1.2.1.2.1, 1.2.1.2.2, 1.2.1.2.3, and 1.2.1.2.4, and 1.2.1.2.5, which will prove $(P \Delta E(H k)),\left(H 1_{i}\right)$, $(H 2),(H 3)$, and $(H 4)$ respectively.

Subsubsubsublemma 1.2.1.2.1: Let $H_{k}\left(n ; a_{1}, \ldots, a_{k}\right)$ be the discrete function defined in the statement of subsublemma 1.2.1, i.e. :

$$
H_{k}\left(n ; a_{1}, \ldots, a_{k}\right):=C T_{x_{1}, \ldots, x_{k}}\left\{\frac{\Phi_{k}\left(x_{1}, \ldots, x_{k}\right)}{\left\{\prod_{i=1}^{k} x_{i}^{a_{i}-1}\left(\bar{x}_{i}\right)^{n+i}\right\} \prod_{1 \leq i<j \leq k}\left(1-x_{i} x_{j}\right)\left(1-\bar{x}_{i} x_{j}\right)}\right\}
$$

where $\Phi_{k}$ is the anti-symmetric polynomial defined in the statement of sublemma 1.2. (In fact all we need is that $\Phi_{k}$ is some anti-symmetric polynomial.) For all $n>k$, and $\left(n ; a_{1}, \ldots, a_{k}\right) \in$ $L_{\text {and_Of_Gog }}$ (in fact whenever $a_{1} \geq \ldots \geq a_{k}$, ) we have that the partial-recurrence equation $\left(P \Delta E\left(H_{k}\right)\right)$, given in the statement of the parent $(s u b)^{3}$ lemma 1.2.1.2, holds.

[ Type 'S12121all $(\mathrm{k}, \mathrm{n})$ :' in ROBBINS, for specific $\mathrm{k}$ and $\mathrm{n}$.]

Proof: Suppose that we have:

$$
a_{1}=a_{2}=\ldots=a_{r_{1}}>a_{r_{1}+1}=\ldots=a_{r_{2}}>\ldots>a_{r_{l-1}+1}=\ldots=a_{r_{l}} \geq 1
$$

where $r_{l}=k$, and $r_{1}, r_{2}-r_{1}, \ldots, r_{l}-r_{l-1}$, are the lengths of maximal blocks of equal components of $a=\left(a_{1}, \ldots, a_{k}\right)$. Putting $c_{i}:=a_{r_{i}}$, for $i=1, \ldots, l$, we have to show that

$$
\begin{gathered}
\left(I-A_{r_{1}}^{-1}\right)\left(I-A_{r_{2}}^{-1}\right) \ldots\left(I-A_{r_{l}}^{-1}\right) H_{k}\left(n ; c_{1}, \ldots, c_{1}, c_{2}, \ldots, c_{2}, \ldots, \ldots, c_{l}, \ldots, c_{l}\right) \\
=H_{k}\left(n-1 ; c_{1}, c_{1}-1, \ldots, c_{1}-1, c_{2}, c_{2}-1, \ldots, c_{2}-1, \ldots, \ldots, c_{l}, c_{l}-1, \ldots, c_{l}-1\right) \quad, \quad(\text { Rodica })
\end{gathered}
$$


where, in the left, the block of $c_{1}$ is $r_{1}$-component-long, the block of $c_{2}$ is $r_{2^{-}}$component-long, $\ldots$, the block of $c_{l}$ is $r_{l}$-component long. In the right side, the $c_{i}-1$ block is $r_{i}-1$-component long, for $i=1, \ldots, l$.

By crucial fact $\aleph_{3}$, the difference between the left and right sides of (Rodica) equals:

$C T_{x_{1}, \ldots, x_{k}}\left[\frac{\left\{\bar{x}_{r_{1}} \bar{x}_{r_{2}} \ldots \bar{x}_{r_{l}}-\left[\bar{x}_{1} \bar{x}_{2} \ldots \bar{x}_{k}\right]\left(x_{2} \ldots x_{r_{1}}\right)\left(x_{r_{1}+2} \ldots x_{r_{2}}\right) \ldots\left(x_{r_{l-1}+2} \ldots x_{r_{l}}\right)\right\} \Phi_{k}\left(x_{1}, \ldots, x_{k}\right)}{\left\{\prod_{i=1}^{k} x_{i}^{a_{i}-1}\left(\bar{x}_{i}\right)^{n+i}\right\} \prod_{1 \leq i<j \leq k}\left(1-x_{i} x_{j}\right)\left(1-\bar{x}_{i} x_{j}\right)}\right]$

(Dave)

In order to prove $(s u b)^{4}$ lemma 1.2.1.2.1, we need to show that the constant-term expression in (Dave) is identically zero. To this end we need the following $(s u b)^{5}$ lemma:

Subsubsubsubsublemma 1.2.1.2.1.1: Let $\operatorname{Jamie}\left(x_{1}, \ldots, x_{k}\right)$ be the polynomial in $\left(x_{1}, \ldots, x_{k}\right)$ inside the braces of (Dave), i.e. the polynomial:

$$
\operatorname{Jamie}\left(x_{1}, \ldots, x_{k}\right):=\prod_{j=1}^{l} \bar{x}_{r_{j}}-\left\{\prod_{i=1}^{k} \bar{x}_{i}\right\}\left\{\prod_{j=1}^{l} \prod_{i=r_{j-1}+2}^{r_{j}} x_{i}\right\} .
$$

$\operatorname{Jamie}\left(x_{1}, \ldots, x_{k}\right)$ can be written as follows (recall that $r_{l}=k$ and we put $r_{0}=0$ ):

$$
\operatorname{Jamie}\left(x_{1}, \ldots, x_{k}\right)=\sum_{\substack{p=2 \\ p \notin\left\{r_{1}+1, r_{2}+1, \ldots, r_{l-1}+1\right\}}}^{k} \operatorname{POL}\left(\widehat{x}_{p-1}, \widehat{x}_{p}\right) \cdot \bar{x}_{p}\left(1-\bar{x}_{p-1} x_{p}\right),
$$

where $\operatorname{POL}\left(\widehat{x}_{p-1}, \widehat{x}_{p}\right)$ is shorthand for: "some polynomial of $\left(x_{1}, \ldots, x_{k}\right)$ that does not depend on the variables $x_{p-1}$ and $x_{p}$ ". (In other words, $P O L\left(\widehat{x}_{p-1}, \widehat{x}_{p}\right)$ is some polynomial in the $k-2$ variables $\left(x_{1}, \ldots, x_{p-2}, x_{p+1}, \ldots, x_{k}\right)$.)

Proof: Since (let $\left.\bar{x}_{0}:=1\right)$

$$
\begin{gathered}
\prod_{i=1}^{k} \bar{x}_{i}=\left\{\prod_{j=1}^{l} \prod_{i=r_{j-1}+1}^{r_{j}} \bar{x}_{i-1}\right\} \cdot \bar{x}_{k}=\left\{\prod_{j=1}^{l} \prod_{i=r_{j-1}+2}^{r_{j}} \bar{x}_{i-1}\right\} \cdot\left\{\prod_{j=1}^{l} \prod_{i=r_{j-1}+1}^{r_{j-1}+1} \bar{x}_{i-1}\right\} \cdot \bar{x}_{k} \\
=\left\{\prod_{j=1}^{l} \prod_{i=r_{j-1}+2}^{r_{j}} \bar{x}_{i-1}\right\} \cdot\left\{\prod_{j=1}^{l} \bar{x}_{r_{j}}\right\},
\end{gathered}
$$

we have that

$$
\begin{gathered}
\operatorname{Jamie}\left(x_{1}, \ldots, x_{k}\right)=\prod_{j=1}^{l} \bar{x}_{r_{j}}-\left\{\prod_{i=1}^{k} \bar{x}_{i}\right\}\left\{\prod_{j=1}^{l} \prod_{i=r_{j-1}+2}^{r_{j}} x_{i}\right\} \\
=\prod_{j=1}^{l} \bar{x}_{r_{j}}-\left\{\prod_{j=1}^{l} \prod_{i=r_{j-1}+2}^{r_{j}}\left(\bar{x}_{i-1} x_{i}\right)\right\} \cdot \prod_{j=1}^{l} \bar{x}_{r_{j}}=\left\{\prod_{j=1}^{l} \bar{x}_{r_{j}}\right\} \cdot\left[1-\prod_{j=1}^{l} \prod_{i=r_{j-1}+2}^{r_{j}}\left(\bar{x}_{i-1} x_{i}\right)\right] .
\end{gathered}
$$

(Marvin) 
We now need the following $(s u b)^{6}$ lemma:

Subsubsubsubsubsublemma 1.2.1.2.1.1.1: Let $U_{j}, j=1, \ldots, l$, be quantities in an associative algebra, then:

$$
1-\prod_{j=1}^{l} U_{j}=\sum_{j=1}^{l}\left\{\prod_{h=1}^{j-1} U_{h}\right\}\left(1-U_{j}\right)
$$

Proof: The series on the right telescopes to the expression on the left. Alternatively, use increasing induction on $l$, starting with the tautologous ground case $l=0$.

Using $(s u b)^{6}$ lemma 1.2.1.2.1.1.1 with

$$
U_{j}=\prod_{i=r_{j-1}+2}^{r_{j}}\left(\bar{x}_{i-1} x_{i}\right)
$$

we get that (Marvin) implies:

$$
\operatorname{Jamie}\left(x_{1}, \ldots, x_{k}\right)=\left\{\prod_{m=1}^{l} \bar{x}_{r_{m}}\right\} \cdot \sum_{j=1}^{l}\left\{\prod_{h=1}^{j-1} \prod_{i=r_{h-1}+2}^{r_{h}}\left(\bar{x}_{i-1} x_{i}\right)\right\} \cdot\left(1-\prod_{i=r_{j-1}+2}^{r_{j}}\left(\bar{x}_{i-1} x_{i}\right)\right) .
$$

We can split (Marvin') yet further apart, with the aid of the following $(s u b)^{6}$ lemma:

Subsubsubsubsubsublemma 1.2.1.2.1.1.2: Let $U_{j},(j=K, \ldots, L)$, be quantities in an associative algebra, then:

$$
1-\prod_{i=K}^{L} U_{i}=\sum_{p=K}^{L}\left(1-U_{p}\right)\left\{\prod_{h=p+1}^{L} U_{h}\right\}
$$

Proof: The sum on the right telescopes to the expression on the left. (Note that it is in the opposite direction to the way in which it happened in 1.2.1.2.1.1.1.) Alternatively, the identity is tautologous when $K=L+1$, and follows by decreasing induction on $K$. This completes the proof of $(s u b)^{6}$ lemma 1.2.1.2.1.1.2.

Going back to $\left(\right.$ Marvin $\left.^{\prime}\right)$, we use the last $(s u b)^{6}$ lemma (1.2.1.2.1.1.2), with $K=r_{j-1}+2, L=r_{j}$, and $U_{i}:=\left(\bar{x}_{i-1} x_{i}\right)$, to rewrite:

$$
\begin{gathered}
\operatorname{Jamie}\left(x_{1}, \ldots, x_{k}\right)=\left\{\prod_{j=1}^{l} \bar{x}_{r_{j}}\right\} \cdot \sum_{j=1}^{l}\left\{\prod_{h=1}^{j-1} \prod_{i=r_{h-1}+2}^{r_{h}}\left(\bar{x}_{i-1} x_{i}\right)\right\} \cdot \sum_{p=r_{j-1}+2}^{r_{j}}\left(1-\bar{x}_{p-1} x_{p}\right) \prod_{i=p+1}^{r_{j}}\left(\bar{x}_{i-1} x_{i}\right) \\
=\sum_{j=1}^{l} \sum_{p=r_{j-1}+2}^{r_{j}}\left\{\prod_{m=1}^{l} \bar{x}_{r_{m}}\right\}\left\{\prod_{h=1}^{j-1} \prod_{i=r_{h-1}+2}^{r_{h}}\left(\bar{x}_{i-1} x_{i}\right)\right\} \cdot\left(1-\bar{x}_{p-1} x_{p}\right)\left\{\prod_{i=p+1}^{r_{j}}\left(\bar{x}_{i-1} x_{i}\right)\right\} .
\end{gathered}
$$


The right side of $\left(\right.$ Marvin $\left.^{\prime \prime}\right)$ is a sum over all $p$ in the range $1 \leq p \leq k$ except that $p=r_{j}+1$, for $j=0, \ldots, l-1$, are omitted (where $r_{0}:=0$.) For each such participating $p$, the summand is $\left(1-\bar{x}_{p-1} x_{p}\right)$ times a product of $x_{i}$ 's and $\bar{x}_{i}$ 's, neither of which is $x_{p}, \bar{x}_{p}, x_{p-1}, \bar{x}_{p-1}$ except for a single $\bar{x}_{p}$, which comes out of the first term of the product $\prod_{i=p+1}^{r_{j}}\left(\bar{x}_{i-1} x_{i}\right)$ in $\left(\right.$ Marvin $\left.^{\prime \prime}\right)$, when $p<r_{j}$ for some $j$, or from the $j$ 'th term of the product $\prod_{j=1}^{l} \bar{x}_{r_{j}}$ in case $p=r_{j}$ for one of the $j$ 's. Thus the polynomial Jamie $\left(x_{1}, \ldots, x_{k}\right)$ can indeed be expressed as claimed in (Herb). This completes the proof of $(s u b)^{5}$ lemma 1.2.1.2.1.1.

Going back to the proof of $(s u b)^{4}$ lemma 1.2.1.2.1, we insert Eq. (Herb) into Eq. (Dave), to get that the expression in (Dave) equals:

$$
\sum_{\substack{p=2 \\ p \notin\left\{r_{1}+1, r_{2}+1, \ldots, r_{l-1}+1\right\}}}^{k} C T_{x_{1}, \ldots, x_{k}}\left\{\frac{\operatorname{POL}\left(\widehat{x}_{p-1}, \widehat{x}_{p}\right) \cdot \bar{x}_{p}\left(1-\bar{x}_{p-1} x_{p}\right) \Phi_{k}\left(x_{1}, \ldots, x_{k}\right)}{\left\{\prod_{i=1}^{k} x_{i}^{a_{i}-1}\left(\bar{x}_{i}\right)^{n+i}\right\} \prod_{1 \leq i<j \leq k}\left(1-x_{i} x_{j}\right)\left(1-\bar{x}_{i} x_{j}\right)}\right\} .
$$

$\left(\right.$ Dave $\left.^{\prime}\right)$

Let $R A T\left(\widehat{x}_{p-1}, \widehat{x}_{p}\right)$ denote a rational function (that possesses a Laurent series) of the $k-2$ variables $\left(x_{1}, \ldots, x_{p-2}, x_{p+1}, \ldots, x_{k}\right)$. We can express the constant-termand of the summand of (Dave $\left.e^{\prime}\right)$ as follows:

$$
\begin{gathered}
\frac{P O L\left(\widehat{x}_{p-1}, \widehat{x}_{p}\right)}{\left\{\prod_{i \neq p-1, p}^{k} x_{i}^{a_{i}-1}\left(\bar{x}_{i}\right)^{n+i}\right\} \prod_{\substack{i \leq i<j \leq k \\
i, j \neq p-1}}\left(1-x_{i} x_{j}\right)\left(1-\bar{x}_{i} x_{j}\right)} \\
\frac{\bar{x}_{p}\left(1-\bar{x}_{p-1} x_{p}\right) \Phi_{k}\left(x_{1}, \ldots, x_{k}\right)}{x_{p-1}^{a_{p-1}-1} x_{p}^{a_{p}-1} \bar{x}_{p-1}^{n+p-1} \bar{x}_{p}^{n+p}\left(1-x_{p-1} x_{p}\right)\left(1-\bar{x}_{p-1} x_{p}\right) \prod_{i=1}^{p-2}\left(1-x_{i} x_{p-1}\right)\left(1-\bar{x}_{i} x_{p-1}\right)\left(1-x_{i} x_{p}\right)\left(1-\bar{x}_{i} x_{p}\right)} \\
\frac{1}{\prod_{j=p+1}^{k}\left(1-x_{p-1} x_{j}\right)\left(1-\bar{x}_{p-1} x_{j}\right)\left(1-x_{p} x_{j}\right)\left(1-\bar{x}_{p} x_{j}\right)} \\
=\frac{R A T\left(\widehat{x}_{p-1}, \widehat{x}_{p}\right) \Phi_{k}\left(x_{1}, \ldots, x_{k}\right)}{x_{p-1}^{a_{p-1}-1} x_{p}^{a_{p}-1} \bar{x}_{p-1}^{n+p-1} \bar{x}_{p}^{n+p-1}\left(1-x_{p-1} x_{p}\right) \prod_{i=1}^{p-2}\left(1-x_{i} x_{p-1}\right)\left(1-\bar{x}_{i} x_{p-1}\right)\left(1-x_{i} x_{p}\right)\left(1-\bar{x}_{i} x_{p}\right)} \\
\frac{1}{\prod_{j=p+1}^{k}\left(1-x_{p-1} x_{j}\right)\left(1-\bar{x}_{p-1} x_{j}\right)\left(1-x_{p} x_{j}\right)\left(1-\bar{x}_{p} x_{j}\right)}
\end{gathered} .
$$

But this constant-termand is manifestly anti-symmetric w.r.t. $x_{p-1} \leftrightarrow x_{p}$, since $\Phi_{k}$ is, and the rest is symmetric, since $a_{p-1}=a_{p}$ (recall that $p \neq r_{j}+1, j=0, \ldots, l-1$.) It follows from crucial fact $\aleph_{1}$ that its constant-term, $C T_{x_{1}, \ldots, x_{k}}$ vanishes. Since every single term in $\left(\right.$ Dave $\left.^{\prime}\right)$ vanishes, it follows that $\left(D_{a v e}\right)$, and hence (Dave) must also vanish. This completes the proof of $(s u b)^{4}$ lemma 1.2.1.2.1.

Subsubsubsublemma 1.2.1.2.2: For $i=1, \ldots, k$, we have, for $\left(n ; a_{1}, \ldots, a_{k}\right) \in \overline{\text {Land_Of_Gog}_{k}}$,

$$
H_{k}\left(n ; a_{1}, \ldots, a_{k}\right)=0 \quad \text { on } \quad a_{i}=k-i \quad \text {. }
$$

[ Type 'S12122(k,n):' in ROBBINS, for specific $\mathrm{k}$ and $\mathrm{n}$. 
Proof: By the definition of $H_{k}$,

$$
\begin{gathered}
H_{k}\left(n ; a_{1}, \ldots, a_{i-1}, k-i, a_{i+1}, \ldots, a_{k}\right)= \\
C T_{x_{1}, \ldots, x_{k}}\left[\frac{\Phi_{k}\left(x_{1}, \ldots, x_{k}\right)}{x_{1}^{a_{1}-1} \cdots x_{i-1}^{a_{i-1}-1} x_{i}^{k-i-1} \cdots x_{k}^{a_{k}-1}} \cdot \prod_{i=1}^{k}\left(\bar{x}_{i}\right)^{-n-i} \prod_{1 \leq i<j \leq k}\left(1-x_{i} x_{j}\right)^{-1}\left(1-\bar{x}_{i} x_{j}\right)^{-1}\right] .
\end{gathered}
$$

For each term of the polynomial $\Phi_{k}$ (see $\left(G_{o g}\right)$ in the statement of sublemma 1.2 for its definition), there are at least $i$ distinct $x$ 's that appear as factors with multiplicity $\geq k-i$. But the denominator of the constant termand has at least $k-i+1 x$ 's in the denominator with exponent $\leq k-i-1$. By the pigeonhole principle, these two sets must have a non-empty intersection. Say $x_{l}$ appears in this intersection. Then, regarded as a Laurent series in $x_{l}$, our constant termand is a positive power of $x_{l}$ times a power series in $x_{l}$. Thus its constant term with respect to $x_{l}$ is zero. This completes the proof of $(s u b)^{4}$ lemma 1.2.1.2.2.

Subsubsubsublemma 1.2.1.2.3: For $\left(n ; n+1, a_{2}, \ldots, a_{k}\right) \in \overline{\operatorname{Land}_{-} O f_{-} G o g_{k}},\left(\right.$ note that $\left.a_{2} \leq n,\right)$

$$
H_{k}\left(n ; n+1, a_{2}, \ldots, a_{k}\right)=0 .
$$

[ Type 'S12123(k,n):' in ROBBINS, for specific $k$ and $n$.]

Proof: By definition,

$$
\begin{gathered}
H_{k}\left(n ; n+1, a_{2}, \ldots, a_{k}\right)= \\
C T_{x_{1}, \ldots, x_{k}}\left[\frac{\Phi_{k}\left(x_{1}, \ldots, x_{k}\right)}{x_{1}^{n} x_{2}^{a_{2}-1} \ldots x_{k}^{a_{k}-1}} \cdot \bar{x}_{1}^{-n-1} \bar{x}_{2}^{-n-2} \ldots \bar{x}_{k}^{-n-k} \prod_{1 \leq i<j \leq k}\left(1-x_{i} x_{j}\right)^{-1}\left(1-\bar{x}_{i} x_{j}\right)^{-1}\right] .
\end{gathered}
$$

(H2) is obvious for $a_{2}=a_{3}=\ldots=a_{k}=0$. It is reasonable to try induction on the size of the $a_{i}$, or more precisely, on their sum. As is often the case when trying to apply induction, we would have to consider points that we do not care about. So let's invite all the vectors $\left(a_{2}, \ldots, a_{k}\right)$ for which the components are all $\leq n$, to be included in the statement of subsubsubsublemma 1.2.1.2.3. Never mind that $\widetilde{M}_{k}$ doesn't make sense unless $a_{2} \geq a_{3} \geq \ldots \geq a_{k}$, right now we are talking about $H_{k}$, for which it is meaningful to consider all such $\left(a_{2}, \ldots, a_{k}\right)$, and we are thus lead to prove the more general statement that $(H 2 a)$ vanishes for all $a_{2}, \ldots, a_{k} \leq n$. By multiplying the numerator by

$$
\bar{x}_{2}^{n+2} \ldots \bar{x}_{k}^{n+k} \prod_{2 \leq i<j \leq k}\left(1-x_{i} x_{j}\right)\left(1-\bar{x}_{i} x_{j}\right)=1+O\left(x_{2}\right)+\ldots+O\left(x_{k}\right)
$$

and using the inductive hypothesis on $a_{2}+\ldots+a_{k}$, it is clear that the assertion that $(H 2 a)$ vanishes for all $a_{2}, \ldots, a_{k} \leq n$ is equivalent to the following simpler statement:

Subsubsubsubsublemma 1.2.1.2.3.1: For all $n \geq k$, and $a_{2}, \ldots, a_{k} \leq n$, the following identity holds. (Recall that for any variable $t$, its bar, $\bar{t}$, stands for $1-t$.)

$$
F_{k}\left(n ; a_{2}, \ldots, a_{k}\right):=C T_{x_{1}, \ldots, x_{k}}\left[\frac{\Phi_{k}\left(x_{1}, \ldots, x_{k}\right)}{x_{1}^{n} \bar{x}_{1}^{n+1} x_{2}^{a_{2}-1} \ldots x_{k}^{a_{k}-1}} \cdot \prod_{i=2}^{k}\left(1-x_{1} x_{i}\right)^{-1}\left(1-\bar{x}_{1} x_{i}\right)^{-1}\right]=0,
$$


where $\Phi_{k}\left(x_{1}, \ldots, x_{k}\right)$ is the polynomial defined in $\left(G_{0}\right)$ (in the statement of sublemma 1.2.)

Proof: We can write $F_{k}\left(n ; a_{2}, \ldots, a_{k}\right)$ as

$$
F_{k}\left(n ; a_{2}, \ldots, a_{k}\right):=C T_{x_{1}}\left[\frac{P\left(x_{1}\right)}{x_{1}^{n} \bar{x}_{1}^{n+1}}\right],
$$

where, $P\left(x_{1}\right)$ is the polynomial in $x_{1}$ given by:

$$
P\left(x_{1}\right):=C T_{x_{2}, \ldots, x_{k}}\left[\frac{\Phi_{k}\left(x_{1}, \ldots, x_{k}\right)}{x_{2}^{a_{2}-1} \ldots x_{k}^{a_{k}-1} \prod_{i=2}^{k}\left(1-x_{1} x_{i}\right)\left(1-\bar{x}_{1} x_{i}\right)}\right] .
$$

Since $P\left(x_{1}\right)$ is obviously anti-symmetric w.r.t. to $x_{1} \rightarrow 1-x_{1}$, it follows by Crucial Fact $\aleph_{2}$, that we would be done once we can prove that the degree of $P\left(x_{1}\right)$ is $\leq 2 n$.

Let's perform a partial-fraction decomposition of the constant-termand of $(H 2 c)$, w.r.t. to $x_{1}$. Since the degree of $\Phi_{k}\left(x_{1}, \ldots, x_{k}\right)$ in $x_{1}$ is $\leq 4 k-3$, we can set:

$$
\frac{\Phi_{k}\left(x_{1}, \ldots, x_{k}\right)}{\prod_{i=2}^{k}\left(1-x_{1} x_{i}\right)\left(1-\bar{x}_{1} x_{i}\right)}=Q_{k}\left(x_{1} ; x_{2}, \ldots, x_{k}\right)+\sum_{i=2}^{k} \frac{B_{i}}{1-x_{1} x_{i}}+\sum_{i=2}^{k} \frac{C_{i}}{1-\bar{x}_{1} x_{i}}
$$

where $B_{i}$ and $C_{i}$ do not depend on $x_{1}$, and $Q_{k}$ is a polynomial of degree $\leq(4 k-3)-(2 k-2)=2 k-1$, in $x_{1}$, with coefficients that are rational functions of $\left(x_{2}, \ldots, x_{k}\right)$. Inserting this into $P\left(x_{1}\right)$ above shows that the contribution to it, due to $Q_{k}$ has degree $\leq 2 k-1<2 n$. We now have to determine the $B_{i}$ 's. By the anti-symmetry of $\Phi_{k}$, we have that $C_{i}=-B_{i} . B_{i}$ is given by the following $(s u b)^{6}$ lemma:

Subsubsubsubsubsublemma 1.2.1.2.3.1. $B_{i}$ in $(H 2 d)$ is given by:

$$
\begin{aligned}
B_{i}= & \pm \frac{\left(1-2 x_{i}\right)\left(1-x_{i}^{2}\right)\left(1-x_{i} \bar{x}_{i}\right) \Phi_{k-2}\left(x_{2}, x_{3}, \ldots, \widehat{x}_{i}, \ldots, x_{k}\right)}{x_{i}^{k+1}} . \\
& \prod_{\substack{j=2 \\
j \neq i}}^{k}\left\{\left(1-x_{i} x_{j}\right)\left(1-\bar{x}_{i} x_{j}\right)\left(1-x_{i} \bar{x}_{j}\right)\left(x_{j}+x_{i}-1\right)\right\}
\end{aligned}
$$

Proof: This is a special case, $R=1$, and $z_{i}=x_{i}$, for all $2 \leq i \leq k$, of subsubsublemma 1.4.1.1 that is proved later, in act IV. This completes the proof of $(s u b)^{6}$ lemma 1.2.1.2.3.1.1.

Since $C_{i}=-B_{i}$, one can write:

$P\left(x_{1}\right)=C T_{x_{2}, \ldots, x_{k}} \frac{Q_{k}\left(x_{1} ; \ldots, x_{k}\right)}{x_{2}^{a_{2}-1} \ldots x_{k}^{a_{k}-1}}+C T_{x_{2}, \ldots x_{k}} \sum_{i=2}^{k} B_{i}\left(\frac{1}{x_{2}^{a_{2}-1} \ldots x_{k}^{a_{k}-1}\left(1-x_{1} x_{i}\right)}-\frac{1}{x_{2}^{a_{2}-1} \ldots x_{k}^{a_{k}-1}\left(1-\bar{x}_{1} x_{i}\right)}\right)$ 
We have already observed that the degree (in $\left.x_{1}\right)$ of the first piece is $\leq 2 n$. Since $B_{i} /\left(1-x_{1} x_{i}\right)$ possesses a Laurent series, we can use crucial fact $\aleph_{4}$ to change the order of the constant-terming, for the $i^{\text {th }}$ term, and get that its contribution is

$$
\begin{gathered}
C T_{x_{2}, \ldots, x_{i}, \ldots, x_{k}}\left\{\frac{ \pm \Phi_{k-2}\left(x_{2}, x_{3}, \ldots, \widehat{x}_{i}, \ldots, x_{k}\right)}{x_{2}^{a_{2}-1} \ldots x_{i-1}^{a_{i-1}-1} x_{i+1}^{a_{i+1}-1} \ldots x_{k}^{a_{k}-1}} .\right. \\
\left.C T_{x_{i}}\left\{\frac{\left(1-2 x_{i}\right)\left(1-x_{i}^{2}\right)\left(1-x_{i} \bar{x}_{i}\right) \prod_{\substack{\begin{subarray}{c}{j=2 \\
j \neq i} }} \\
{k}\end{subarray}}\left[\left(1-x_{i} x_{j}\right)\left(1-\bar{x}_{i} x_{j}\right)\left(1-x_{i} \bar{x}_{j}\right)\left(x_{j}+x_{i}-1\right)\right]}{x_{i}^{a_{i}+k}} \cdot \frac{1}{\left(1-x_{i} x_{1}\right)}\right\}\right\} .
\end{gathered}
$$

Using the Maclaurin expansion (in $x_{i}$ )

$$
\left(1-x_{1} x_{i}\right)^{-1}=\sum_{r=0}^{\infty} x_{1}^{r} x_{i}^{r}
$$

and expanding the numerator of the last constant-termand (w.r.t. $x_{i}$ ) in powers of $x_{i}$ :

$$
\sum_{r=0}^{4 k-3} L_{r}\left(x_{2}, \ldots, \widehat{x}_{i}, \ldots, x_{k}\right) x_{i}^{r}
$$

multiplying out, and then extracting the coefficient of $x_{i}^{a_{i}+k}$, shows that the inner constant-term (w.r.t. $x_{i}$, ) can be written as:

$$
\sum_{r=0}^{\min \left(a_{i}+k, 4 k-3\right)} L_{r}\left(x_{2}, \ldots, \widehat{x}_{i}, \ldots, x_{k}\right) x_{1}^{a_{i}+k-r}
$$

which is a polynomial of degree $\leq k+a_{i} \leq n+n=2 n$ in $x_{1}$, with coefficients that are polynomials in the remaining variables $\left(x_{2}, \ldots, \widehat{x}_{i}, \ldots, x_{k}\right)$. Taking the outer constant-term does not change this fact, except that now we arrive at a polynomial in $x_{1}$ with numerical coefficients. The same argument (with $\bar{x}_{1}$ replaced by $x_{1}$ ) holds for the term $-B_{i} /\left(1-\bar{x}_{1} x_{i}\right)$.

Since each and every contribution to $P\left(x_{1}\right)$ has degree $\leq 2 n$, the same must be true of $P\left(x_{1}\right)$ itself, and hence completes the proof of subsubsubsubsublemma 1.2.1.2.3.1.

This completes the proof of subsubsubsublemma 1.2.1.2.3 $\square$.

Subsubsubsublemma 1.2.1.2.4: For $\left(k ; k, a_{2}, \ldots, a_{k}\right) \in \overline{\text { Land_Of_Gog }_{k}}$,

$$
H_{k}\left(k ; k, a_{2}, \ldots, a_{k}\right)=H_{k-1}\left(k ; a_{2}, \ldots, a_{k}\right) .
$$

[ Type 'S12124(k):' in ROBBINS, for specific k.]

Proof: From the definition of $H_{k}$,

$H_{k}\left(k ; k, a_{2}, \ldots, a_{k}\right)=C T_{x_{1}, \ldots, x_{k}}\left\{\frac{\Phi_{k}\left(x_{1}, \ldots, x_{k}\right)}{x_{1}^{k-1} x_{2}^{a_{2}-1} \ldots x_{k}^{a_{k}-1} \bar{x}_{1}^{k+1} \ldots \bar{x}_{k}^{2 k}} \prod_{1 \leq i<j \leq k}\left(1-x_{i} x_{j}\right)^{-1}\left(1-\bar{x}_{i} x_{j}\right)^{-1}\right\}$. 
Recall, from the definition of $\overline{\operatorname{Land}_{-} O f_{-} G o g_{k}}$ (given at the beginning of the proof of 1.2.1), that $k>a_{2}$. Let's look at the contributions of the various terms of $\Phi_{k}$ (defined in $\left(\operatorname{Gog}_{1}\right)$ ), to the constant term (1.2.1.2.4a). Because of the low exponents $(<k)$ of the $x_{i}$ 's that appear in the denominator of (1.2.1.2.4a), it is clear that only those $g=(\pi, \varepsilon)$ for which all the $\varepsilon_{i}$ 's equal -1 , have any hope of contributing a non-zero term. It follows that (note that we "lose" a factor of $(-1)^{k}$ due to fixing all the $k \varepsilon_{i}$ 's to be -1$)$ :

$H_{k}\left(k ; k, a_{2}, \ldots, a_{k}\right)=C T_{x_{1}, \ldots, x_{k}}\left\{\frac{\Psi_{k}\left(x_{1}, \ldots, x_{k}\right)}{x_{1}^{k-1} x_{2}^{a_{2}-1} \ldots x_{k}^{a_{k}-1} \bar{x}_{1}^{k+1} \ldots \bar{x}_{k}^{2 k}} \prod_{1 \leq i<j \leq k}\left(1-\bar{x}_{i} x_{j}\right)^{-1}\left(1-x_{i} x_{j}\right)^{-1}\right\}$

where

$$
\Psi_{k}\left(x_{1}, \ldots, x_{k}\right)=\sum_{\pi \in \mathcal{S}_{k}} \operatorname{sgn}(\pi) \cdot \pi\left[\prod_{i=1}^{k} x_{i}^{k-i} \bar{x}_{i}^{k} \prod_{1 \leq i<j \leq k}\left(1-\bar{x}_{i} x_{j}\right)\left(1-x_{i} x_{j}\right)\right]
$$

Even now, there are lots of terms of $\Psi_{k}$ that do not contribute to the constant term of (1.2.1.2.4b). All those terms corresponding to $\pi$ for which $\pi(1) \neq 1$, will have one of the $x_{j}$, for $j>1$ raised to a power $k-1$, which will introduce a factor of $x_{j}$ and shatter any hope for a non-zero contribution to the constant term. So let's write:

$$
\begin{gathered}
\Psi_{k}\left(x_{1}, \ldots, x_{k}\right)=x_{1}^{k-1} \bar{x}_{1}^{k}\left(1-\bar{x}_{1} x_{2}\right) \ldots\left(1-\bar{x}_{1} x_{k}\right)\left(1-x_{1} x_{2}\right) \ldots\left(1-x_{1} x_{k}\right) \Psi_{k-1}\left(x_{2}, \ldots, x_{k}\right)+\text { GARBAGE } \\
=x_{1}^{k-1} \bar{x}_{1}^{k}\left(\bar{x}_{2} \ldots \bar{x}_{k}\right)(1) \ldots(1) \Psi_{k-1}\left(x_{2}, \ldots, x_{k}\right)+O\left(x_{1}^{k}\right)+\text { GARBAGE }
\end{gathered}
$$

where $G A R B A G E$ denotes terms that clearly contribute 0 to the constant term, and in the second equality we have used that $\left(1-\bar{x}_{1} x_{2}\right) \ldots\left(1-\bar{x}_{1} x_{k}\right)=\left(\bar{x}_{2}+x_{1} x_{2}\right) \ldots\left(\bar{x}_{k}+x_{1} x_{k}\right)=\bar{x}_{2} \ldots \bar{x}_{k}+O\left(x_{1}\right)$.

Thus,

$$
\begin{gathered}
H_{k}\left(k ; k, a_{2}, \ldots, a_{k}\right)=C T_{x_{2}, \ldots, x_{k}} C T_{x_{1}}\left\{\frac{x_{1}^{k-1} \bar{x}_{1}^{k} \bar{x}_{2} \ldots \bar{x}_{k} \Psi_{k-1}\left(x_{2}, \ldots, x_{k}\right)}{x_{1}^{k-1} x_{2}^{a_{2}-1} \ldots x_{k}^{a_{k}-1} \bar{x}_{1}^{k+1} \ldots \bar{x}_{k}^{2 k}} .\right. \\
\left.\prod_{1<j \leq k}\left(1-x_{1} x_{j}\right)^{-1}\left(1-\bar{x}_{1} x_{j}\right)^{-1} \prod_{2 \leq i<j \leq k}\left(1-x_{i} x_{j}\right)^{-1}\left(1-\bar{x}_{i} x_{j}\right)^{-1}\right\} \\
=C T_{x_{2}, \ldots, x_{k}}\left\{\frac{\bar{x}_{2} \ldots \bar{x}_{k} \Psi_{k-1}\left(x_{2}, \ldots, x_{k}\right)}{x_{2}^{a_{2}-1} \ldots x_{k}^{a_{k}-1} \bar{x}_{2}^{k+2} \ldots \bar{x}_{k}^{2 k}} \cdot \prod_{2 \leq i<j \leq k}\left(1-x_{i} x_{j}\right)^{-1}\left(1-\bar{x}_{i} x_{j}\right)^{-1} .\right. \\
\left.C T_{x_{1}}\left\{\left(1 / \bar{x}_{1}\right) \prod_{1<j \leq k}\left(1-x_{1} x_{j}\right)^{-1}\left(1-\bar{x}_{1} x_{j}\right)^{-1}\right\}\right\} \\
=C T_{x_{2}, \ldots, x_{k}}\left\{\frac{\Psi_{k-1}\left(x_{2}, \ldots, x_{k}\right)}{x_{2}^{a_{2}-1} \ldots x_{k}^{a_{k}-1} \bar{x}_{2}^{k+1} \ldots \bar{x}_{k}^{2 k-1}} \cdot \prod_{2 \leq i<j \leq k}\left(1-x_{i} x_{j}\right)^{-1}\left(1-\bar{x}_{i} x_{j}\right)^{-1} \cdot 1\right\} .
\end{gathered}
$$


I claim that this equals $H_{k-1}\left(k ; a_{2}, \ldots, a_{k}\right)$. By the definition $\left(H_{k}\right)$ given in the statement of subsublemma 1.2.1, we have:

$$
H_{k-1}\left(k ; a_{2}, \ldots, a_{k}\right):=C T_{x_{2}, \ldots, x_{k}}\left\{\frac{\Phi_{k-1}\left(x_{2}, \ldots, x_{k}\right)}{\left\{\prod_{i=2}^{k} x_{i}^{a_{i}-1}\left(\bar{x}_{i}\right)^{(k-1)+i}\right\} \prod_{2 \leq i<j \leq k}\left(1-x_{i} x_{j}\right)\left(1-\bar{x}_{i} x_{j}\right)}\right\},
$$

Since $k>a_{2} \geq a_{3} \ldots \geq a_{k}$, a parallel argument can be given to show that the right side of $\left(H_{k-1}(k)\right)$ equals the right side of $\left(H_{k}(k)\right)$. This completes the proof of subsubsubsublemma 1.2.1.2.4.

Subsubsubsublemma 1.2.1.2.5: $(H 4)$ in the statement of subsubsublemma 1.2 .1 .2 holds.

This completes the proof of subsubsublemma 1.2.1.2.

Subsubsublemma 1.2.1.3: Let $k \geq 1$ and for any vector of integers $a=\left(a_{1}, \ldots, a_{k}\right)$, define the partial difference operator $P^{(a)}$ by:

$$
P^{(a)}\left(A_{1}, \ldots, A_{k}\right):=\prod_{\left\{i \mid a_{i}-a_{i+1}>0\right\}}\left(I-A_{i}^{-1}\right) \quad,
$$

where we declare that $a_{k+1}=0$. There is a unique sequence of discrete functions $Y_{k}\left(n ; a_{1}, \ldots, a_{k}\right)$, defined on $\overline{\operatorname{Land\_ Of\_ Gog}_{k}}$ that satisfies the following partial recurrence

$$
\begin{gathered}
P^{(a)}\left(A_{1}, \ldots, A_{k}\right) Y_{k}\left(n ; a_{1}, \ldots, a_{k}\right) \\
=Y_{k}\left(n-1 ; a_{1}, \min \left(a_{1}-1, a_{2}\right), \ldots, \min \left(a_{k-1}-1, a_{k}\right)\right),
\end{gathered}
$$

valid for $n>k$ and for $\left(n ; a_{1}, \ldots, a_{k}\right) \in L_{\text {Lnd_Of_Gog }}$, and the following initial/boundary conditions for $\left(n ; a_{1}, \ldots, a_{k}\right) \in \overline{\text { Land_Of_Gog }_{k}}$ :

$$
\begin{gathered}
Y_{k}\left(n ; a_{1}, \ldots, a_{k}\right)=0 \quad \text { on } \quad a_{i}=k-i \\
Y_{k}\left(n ; n+1, a_{2}, \ldots, a_{k}\right)=0 \\
Y_{k}\left(k ; k, a_{2}, \ldots, a_{k}\right)=Y_{k-1}\left(k ; a_{2}, \ldots, a_{k}\right)
\end{gathered}
$$

and, finally the "Adam" condition

$$
Y_{1}(1 ; 1)=1 \quad .
$$

[ Type 'S1213(k,n):' in ROBBINS, for specific $k$ and $n$.

Proof: Eq. $P \Delta E\left(Y_{k}\right)$ can be rewritten such that $Y_{k}\left(n ; a_{1}, \ldots, a_{k}\right)$ remains on the left side, and all the rest goes to the right side. The right side involves either points $\left(n ; b_{1}, \ldots, b_{k}\right)$ that belong to $\overline{\text { Land_Of_Gog }}$, with $b_{1}+\ldots+b_{k}<a_{1}+a_{2}+\ldots+a_{k}$, so they were already computed before, or points that belong to the boundary, for which the boundary conditions are used. Note that the reason that it was possible to impose the extra restriction that $n=k=a_{1} \Rightarrow a_{2}<k$, in the definition of $\overline{\operatorname{Land}_{-} O f_{-} G o g_{k}}$, was that when we use the local recurrence $\left(P \Delta E\left(\widetilde{M}_{k}\right)\right)$ to compute 
$\widetilde{M}_{k}$, starting with $n=k+1$, the right hand side of $\left(P \Delta E\left(\widetilde{M}_{k}\right)\right)$ only requires knowledge of $\widetilde{M}_{k}$, when $n=k=a_{1}$, for those $a$ 's for which $a_{2}<k$, since even if $a_{2}=k$ on the left side, the evaluation at the right is at $\widetilde{M}_{k}(k ; k, \min (k-1, k), \ldots)=\widetilde{M}_{k}(k ; k, k-1, \ldots$,$) . This completes the proof of$ subsubsublemma 1.2.1.3.

Combining subsubsublemmas 1.2.1.1 - 3 yields subsublemma 1.2.1.

Going back to the proof of sublemma 1.2, note that $m_{k}(n)$, the total number of $n \times k$-Gog trapezoids, equals $\widetilde{M}_{k}(n+1 ; n+1, n+1, \ldots, n+1)$, as follows directly from the definition $\left(\widetilde{M}_{k}\right)$ of $\widetilde{M}_{k}$. By subsublemma 1.2.1, this equals $H_{k}(n+1 ; n+1, n+1, \ldots, n+1)$ which coincides with the right side of (GogTotal). This completes the proof of sublemma 1.2. $\square$.

\section{INTERACT}

We have now established constant term expressions both for $b_{k}(n)$, the number of $n \times k$ Magog trapezoids, given by (MagogTotal) in sublemma 1.1, and for $m_{k}(n)$, the number of $n \times k$ Gog trapezoids, given by (GogTotal) in sublemma 1.2. It would have been nice if the constant-termands were the same. Unfortunately, they are not. In order to prove that the constant terms are, we have to resort to the omnipotent Stanton-Stembridge trick once again, only now w.r.t. to the action of the group of signed permutations, $W\left(\mathcal{B}_{k}\right)$. Justifying its use, however, will require many more pages. It would be necessary first to convert the constant-term expressions to "residue" expressions.

Sublemma 1.1': The total number of $n \times k$ - Magog trapezoids, $b_{k}(n)$, is given by the following iterated-residue expression:

$$
b_{k}(n)=\operatorname{Res}_{x_{1}, \ldots, x_{k}}\left\{\frac{\Delta_{k}\left(x_{1}, \ldots, x_{k}\right)}{\prod_{i=1}^{k} x_{i}^{n+k-i}\left(\bar{x}_{i}\right)^{n+k+1} \prod_{1 \leq i<j \leq k}\left(1-x_{i} x_{j}\right)}\right\},
$$

where,

$$
\Delta_{k}\left(x_{1}, \ldots, x_{k}\right):=\prod_{i=1}^{k}\left(1-2 x_{i}\right) \prod_{1 \leq i<j \leq k}\left(x_{j}-x_{i}\right)\left(x_{j}+x_{i}-1\right)
$$

Sublemma 1.2': The total number of $n \times k-$ Gog trapezoids, $m_{k}(n)$, is given by the following iterated-residue expression:

$$
m_{k}(n)=\operatorname{Res}_{x_{1}, \ldots, x_{k}}\left[\frac{\Phi_{k}\left(x_{1}, \ldots, x_{k}\right)}{\left\{\prod_{i=1}^{k} x_{i}^{n+1}\left(\bar{x}_{i}\right)^{n+i+1}\right\} \prod_{1 \leq i<j \leq k}\left(1-x_{i} x_{j}\right)\left(1-\bar{x}_{i} x_{j}\right)}\right],
$$

$($ GogTotal')

where the polynomial $\Phi_{k}\left(x_{1}, \ldots, x_{k}\right)$ is defined by:

$$
\Phi_{k}\left(x_{1}, \ldots, x_{k}\right)=(-1)^{k} \sum_{g \in W\left(\mathcal{B}_{k}\right)} \operatorname{sgn}(g) \cdot g\left[\prod_{i=1}^{k} \bar{x}_{i}^{k-i} x_{i}^{k} \prod_{1 \leq i<j \leq k}\left(1-x_{i} \bar{x}_{j}\right)\left(1-\bar{x}_{i} \bar{x}_{j}\right)\right]
$$

Proof of Sublemmas 1.1' and 1.2': Use Crucial fact $\aleph_{6}$. 


\section{Act III. (MagogTotal') IS UNFAZED BY THE SIGNED PERMUTATIONS' ACTION}

Talk no more so exceeding proudly; let not arrogancy come out of your mouth: for the Lord is a God of knowledge, and by him actions are weighed.

Sublemma 1.3 : Let $n \geq k \geq 1$ and let $f_{n, k}(x)=f_{n, k}\left(x_{1}, \ldots, x_{k}\right)$ be the residuand of $\left(\right.$ MagogTotal $\left.^{\prime}\right)$ :

$$
f_{n, k}\left(x_{1}, \ldots, x_{k}\right):=\frac{\Delta_{k}\left(x_{1}, \ldots, x_{k}\right)}{\prod_{i=1}^{k}\left(\bar{x}_{i}\right)^{n+k+1} x_{i}^{n+k-i} \prod_{1 \leq i<j \leq k}\left(1-x_{i} x_{j}\right)},
$$

where $\Delta_{k}\left(x_{1}, \ldots, x_{k}\right)$ is the polynomial defined in (Delta) (in the statement of sublemma 1.2). Let $g=(\pi, \varepsilon)$ be any signed permutation in $W\left(\mathcal{B}_{k}\right)$; then

$$
\operatorname{Res}_{x_{1}, \ldots, x_{k}}\left[g f_{n, k}(x)\right]=\operatorname{Res}_{x_{1}, \ldots, x_{k}}\left[f_{n, k}(x)\right] \quad\left(=b_{k}(n)\right)
$$

[ Type 'S13(k,n):' in ROBBINS, for specific $k$ and $n$.

Proof: Recall that a signed permutation $g=(\pi, \varepsilon)$ acts on any rational function $f(x)$ of $x=$ $\left(x_{1}, \ldots, x_{k}\right)$, by $g f(x)=f(\varepsilon(\pi(x)))$. By renaming the variables, and replacing $\pi^{-1}$ by $\pi$, one finds that the statement of sublemma 1.3 is equivalent to:

Sublemma 1.3' : Let $f_{n, k}(x)=f_{n, k}\left(x_{1}, \ldots, x_{k}\right)$ be the residuand of (MagogTotal'), reproduced just above. Let $\pi$ be any permutation of $\{1, \ldots, k\}$, and let $\varepsilon=\left(\varepsilon_{1}, \ldots, \varepsilon_{k}\right)$ be any sign-assignment, then

$$
\operatorname{Res}_{x_{\pi(1)}, \ldots, x_{\pi(k)}}\left[f_{n, k}\left(\varepsilon_{1}\left(x_{1}\right), \ldots, \varepsilon_{k}\left(x_{k}\right)\right)\right]=\operatorname{Res}_{x_{1}, \ldots, x_{k}}\left[f_{n, k}\left(x_{1}, \ldots, x_{k}\right)\right]
$$

The proof will be by induction on the number of $\varepsilon_{i}$ 's that are equal to -1 . If there are no -1 's in $\varepsilon$, then $1.3^{\prime}$ follows immediately from crucial fact $\aleph_{4}^{\prime}$, since then the residuand $f_{n, k}\left(x_{1}, \ldots, x_{k}\right)$ has a Laurent series. Otherwise, let $u$ be the smallest integer $i,(1 \leq i \leq k)$ such that $\varepsilon_{\pi(i)}=-1$. The inductive step is provided by the following subsublemma, that asserts that it is permissible to change $\varepsilon_{\pi(u)}$ from -1 to +1 inside the residuand of the iterated residue on the left side of $\left(1.3^{\prime}\right)$, without affecting its value.

Subsublemma 1.3.1: Let $f_{n, k}\left(x_{1}, \ldots, x_{k}\right)$ (where, as always, $n \geq k \geq 1$ ) be the residuand of (MagogTotal'), let $\pi$ be any permutation of $\{1, \ldots, k\}$, and let $\varepsilon=\left(\varepsilon_{1}, \ldots, \varepsilon_{k}\right)$ be any signassignment with at least one $\varepsilon_{i}$ equal to -1 . Let $u$ be the smallest $i(1 \leq i \leq k)$ such that $\varepsilon_{\pi(i)}=-1$. (i.e., we have $\varepsilon_{\pi(1)}=\ldots=\varepsilon_{\pi(u-1)}=+1$, and $\varepsilon_{\pi(u)}=-1$.) Then:

$\operatorname{Res}_{x_{\pi(1)}, \ldots, x_{\pi(k)}}\left[f_{n, k}\left(\varepsilon_{1}\left(x_{1}\right), \ldots, x_{\pi(u)}, \ldots, \varepsilon_{k}\left(x_{k}\right)\right)\right]=\operatorname{Res}_{x_{\pi(1)}, \ldots, x_{\pi(k)}}\left[f_{n, k}\left(\varepsilon_{1}\left(x_{1}\right), \ldots, \bar{x}_{\pi(u)}, \ldots, \varepsilon_{k}\left(x_{k}\right)\right)\right]$

[ Type 'S131 $(\mathrm{k}, \mathrm{n})$ :' in ROBBINS, for specific $\mathrm{k}$ and $\mathrm{n}$.

Proof: Let's abbreviate $R:=\pi(u)$, and $z_{i}:=\varepsilon_{i}\left(x_{i}\right)(1 \leq i \leq k, i \neq R$.) Recall that this means that $z_{i}=x_{i}$ if $\varepsilon_{i}=+1$, and $z_{i}=\bar{x}_{i}=1-x_{i}$ if $\varepsilon_{i}=-1$. By the $W\left(\mathcal{B}_{k}\right)$-antisymmetry of $\Delta_{k}(x)$, 
we have

$$
f_{n, k}\left(\varepsilon_{1}\left(x_{1}\right), \ldots, x_{R}, \ldots, \varepsilon_{k}\left(x_{k}\right)\right)=\frac{1}{x_{R}^{n+k-R} \bar{x}_{R}^{n+k+1}} \prod_{\substack{j=1 \\ j \neq R}}^{k} \frac{1}{z_{j}^{n+k-j} \bar{z}_{j}^{n+k+1}} \prod_{\substack{1 \leq r<s \leq k \\ r, s \neq R}} \frac{1}{\left(1-z_{r} z_{s}\right)} \cdot\left\{\frac{ \pm \Delta_{k}\left(x_{1}, \ldots, x_{k}\right)}{\prod_{\substack{k \\ i \neq 1 \\ i \neq R}}\left(1-z_{i} x_{R}\right)}\right\}
$$

The next step is to perform a partial-fraction decomposition, with respect to the variable $x_{R}$, of the fraction inside (Jane)'s braces.

\section{Subsubsublemma 1.3.1.1:}

$$
\frac{ \pm \Delta_{k}\left(x_{1}, \ldots, x_{k}\right)}{\prod_{\substack{i=1 \\ i \neq R}}^{k}\left(1-z_{i} x_{R}\right)}=P_{R}+\sum_{\substack{i=1 \\ i \neq R}}^{k} \frac{B_{i}}{1-z_{i} x_{R}}
$$

where $P_{R}$ is a polynomial of degree $k$ in $x_{R}$, with coefficients that are rational functions of the other variables $\left(x_{1}, \ldots, x_{R-1}, x_{R+1}, \ldots, x_{k}\right)$, and

$$
\begin{aligned}
B_{i}=\frac{ \pm\left(z_{i}-2\right)\left(1-2 z_{i}\right)\left(1-z_{i}^{2}\right)\left(1-z_{i} \bar{z}_{i}\right) \Delta_{k-2}\left(x_{1}, \ldots, \widehat{x}_{i}, \ldots, \widehat{x}_{R}, \ldots, x_{k}\right)}{z_{i}^{k+1}} . \\
\left\{\prod_{\substack{j=1 \\
j \neq i, R}}^{k}\left(1-z_{i} z_{j}\right)\left(1-z_{i} \bar{z}_{j}\right)\left(z_{j}+z_{i}-1\right)\right\} .
\end{aligned}
$$

[ Type 'S1311 $(k, R):$ ' in ROBBINS, for specific $k$ and R.]

Proof: By Freshman Calculus (or rather the algebraic part of it), we know that the left side of (Tamar), when viewed as a rational function of $x_{R}$, can be written as on its right side, for some polynomial $P_{R}$, and constants (in the present context, i.e. quantities not involving $x_{R}$ ) $B_{i}$. The degree of $\Delta_{k}$, in $x_{R}$ (or for that matter, in any variable), from its definition in (Delta), is $2 k-1$, and the degree of the denominator of the left side of (Tamar) is $(k-1)$. Hence the degree of $P_{R}$, in $x_{R}$ is $(2 k-1)-(k-1)=k$. The coefficients of $P_{R}$, as a polynomial of $x_{R}$, are certain rational functions of the remaining variables whose exact form does not interest us. The exact form of the $B_{i}$ 's should interest us, since it is used heavily in the proof. So let's brace ourselves and embark on partial-fractioning.

Multiplying both sides of (Tamar) by the denominator of its left side yields the equation:

$$
\pm \Delta_{k}\left(x_{1}, \ldots, x_{k}\right)=\left\{\prod_{\substack{j=1 \\ j \neq R}}^{k}\left(1-z_{j} x_{R}\right)\right\} P_{R}+\sum_{\substack{i=1 \\ i \neq R}}^{k}\left\{\prod_{\substack{j=1 \\ j \neq R, i}}^{k}\left(1-z_{j} x_{R}\right)\right\} B_{i}
$$

To decipher $B_{i}(1 \leq i \leq k, i \neq R)$, we plug in $x_{R}=1 / z_{i}$ on both sides of (Tamar') to get:

$$
B_{i}=\frac{ \pm\left.\Delta_{k}\left(x_{1}, \ldots, x_{k}\right)\right|_{x_{R}=1 / z_{i}}}{\left\{\prod_{\substack{j=1 \\ j \neq R, i}}^{k}\left(1-z_{j} / z_{i}\right)\right\}}=\frac{ \pm\left. z_{i}^{k-2} \Delta_{k}\left(x_{1}, \ldots, x_{k}\right)\right|_{x_{R}=1 / z_{i}}}{\left\{\prod_{\substack{j=1 \\ j \neq R, i}}^{k}\left(z_{i}-z_{j}\right)\right\}}
$$




\section{Subsubsubsublemma 1.3.1.1.1:}

$$
\begin{gathered}
\left.\Delta_{k}\left(x_{1}, x_{2}, \ldots, x_{k}\right)\right|_{x_{R}=1 / z_{i}}= \pm \frac{\left(z_{i}-2\right)\left(1-2 z_{i}\right)\left(1-z_{i}^{2}\right)\left(1-z_{i} \bar{z}_{i}\right) \cdot \Delta_{k-2}\left(x_{1}, x_{2}, x_{3}, \ldots, \widehat{x}_{i}, \ldots, \widehat{x}_{R}, \ldots, x_{k}\right)}{z_{i}^{2 k-1}} \\
\prod_{\substack{j=1 \\
j \neq i, R}}^{k}\left\{\left(1-z_{i} z_{j}\right)\left(1-z_{i} \bar{z}_{j}\right)\left(z_{j}-z_{i}\right)\left(z_{j}+z_{i}-1\right)\right\}
\end{gathered}
$$

[ Type 'S13111(k,R,i):' in ROBBINS, for specific $k, R$ and i.]

Proof: This follows routinely from the definition (Delta) of $\Delta_{k}$. $\square$.

Plugging (1.3.1.1.1) into (PP1), and cancelling out $\prod_{\substack{j=1 \\ j \neq i, R}}^{k}\left(z_{j}-z_{i}\right)$ from top and bottom, finishes up the proof of $(s u b)^{3}$ lemma 1.3.1.1.

For the rest of the proof of $(s u b)^{2}$ lemma 1.3.1, we need some notation. Let $P O L$ stand for "some polynomial of $\left(x_{1}, \ldots, x_{k}\right)$ ", and let $R A T$ stand for "some rational function of $\left(x_{1}, \ldots, x_{k}\right)$ ". $\operatorname{POL}\left(\widehat{x}_{R}\right)$ stands for such a polynomial that does not involve $x_{R} \operatorname{POLRAT}\left(x_{R} ; x_{1}, \ldots, \widehat{x}_{R}, \ldots, x_{k}\right)$, or $\operatorname{POLRAT}\left(x_{R} ; \ldots\right)$ for short, stands for a polynomial in $x_{R}$ with coefficients that are rational functions of the rest of the variables. POLRAT $\left(x_{i} ; \widehat{x}_{R}\right)$ stands for a polynomial in $x_{i}$ with coefficients that are rational function of the remaining variables and that does not involve $x_{R}$.

Inserting (Tamar) (in the statement of 1.3.1.1) into (Jane) is going to yield the following $(s u b)^{3}$ lemma:

\section{Subsubsublemma 1.3.1.2:}

$$
f_{n, k}\left(\operatorname{epsilon}_{1}\left(x_{1}\right), \ldots, x_{R}, \ldots, \varepsilon_{k}\left(x_{k}\right)\right)=\frac{\widetilde{P}_{R}}{x_{R}^{n+k-R} \bar{x}_{R}^{n+k+1}}+\sum_{\substack{i=1 \\ i \neq R}}^{k} \frac{\widetilde{B}_{i}}{1-z_{i} x_{R}}
$$

where $\widetilde{P}_{R}$ is a polynomial of degree $k$ in $x_{R}$, with coefficients that are rational functions of the rest of the variables, and $\widetilde{B}_{i}$ can be written as follows.

$$
\begin{gathered}
\widetilde{B}_{i}=\frac{\operatorname{POL}\left(\widehat{x}_{R}\right)}{x_{R}^{n+k-R} \bar{x}_{R}^{n+k+1} z_{i}^{n+2 k-i+1} \bar{z}_{i}^{n+k+1}} \cdot \prod_{\substack{j=1 \\
j \neq i, R}}^{k} \frac{1}{z_{j}^{n+k-j} \bar{z}_{j}^{n+k+1}} \cdot \prod_{\substack{1 \leq r<s \leq k \\
r, s \neq R, i}} \frac{1}{\left(1-z_{r} z_{s}\right)} \\
=\frac{\operatorname{POLRAT}\left(x_{i} ; \widehat{x}_{R}\right)}{x_{R}^{n+k-R} \bar{x}_{R}^{n+k+1} z_{i}^{n+2 k-i+1} \bar{z}_{i}^{n+k+1}} \cdot
\end{gathered}
$$

[ Type 'S1312(k,n,eps, R) :' in ROBBINS, for specific k,n, eps and R.]

Proof: Insert (Tamar) into (Jane), bringing the quantity in front of the sum inside, and for each $B_{i}$ split the double product:

$$
\prod_{\substack{1 \leq r<s \leq k \\ r, s \neq R}} \frac{1}{\left(1-z_{r} z_{s}\right)}=\prod_{\substack{1 \leq r<s \leq k \\ r, s \neq R, i}} \frac{1}{\left(1-z_{r} z_{s}\right)} \cdot \prod_{\substack{j=1 \\ j \neq i, R}}^{k} \frac{1}{\left(1-z_{j} z_{i}\right)}
$$


and cancel out $\prod_{\substack{j \neq i, R \\ j \neq i}}^{k}\left(1-z_{j} z_{i}\right)$ on the top and bottom. This completes the proof of $(s u b)^{3}$ lemma 1.3.1.2.

We will say that a rational function $h=h\left(x_{1}, \ldots, x_{k}\right)$ has property (Hadas) if:

$$
\operatorname{Res}_{x_{\pi(1)}, \ldots, x_{\pi(k)}}[h]=\operatorname{Res}_{x_{\pi(1)}, \ldots, x_{\pi(k)}}\left[h\left(x_{R} \rightarrow \bar{x}_{R}\right)\right],
$$

where $h\left(x_{R} \rightarrow \bar{x}_{R}\right)$ is the rational function obtained from $h$ by replacing the variable $x_{R}$ by $\bar{x}_{R}$ $\left(=1-x_{R} \cdot\right)$

The statement of subsublemma 1.3.1 is equivalent to saying that

$$
f_{n, k}\left(\varepsilon_{1}\left(x_{1}\right), \ldots, x_{R}, \ldots, \varepsilon_{k}\left(x_{k}\right)\right)
$$

has property (Hadas). Property (Hadas) is obviously additive (since the action of taking residue, and performing $x_{R} \rightarrow \bar{x}_{R}$ are), so in order to complete the proof of 1.3 .1 (and hence of 1.3), it would suffice to show that each term in (Celia) has property (Hadas). This will be accomplished by the following two subsubsublemmas $(1.3 .1 .3-4$.)

Subsubsublemma 1.3.1.3: Let $\widetilde{P}_{R}$ be as in the statement of 1.3 .1 .2 , i.e. a polynomial of degree $k$ in $x_{R}$ with coefficients that are rational functions of the remaining variables. Then $\frac{\widetilde{P}_{R}}{x_{R}^{n+k-R} \bar{x}_{R}^{n+k+1}}$ has property (Hadas):

$$
\operatorname{Res}_{x_{\pi(1)}, \ldots, x_{\pi(k)}}\left[\frac{\widetilde{P}_{R}}{x_{R}^{n+k-R} \bar{x}_{R}^{n+k+1}}\right]=\operatorname{Res}_{x_{\pi(1)}, \ldots, x_{\pi(k)}}\left[\frac{\widetilde{P}_{R}}{x_{R}^{n+k-R} \bar{x}_{R}^{n+k+1}}\left(x_{R} \rightarrow \bar{x}_{R}\right)\right] \quad \cdot \quad\left(\operatorname{Hadas}_{1}\right)
$$

[ Type 'S1313(k,n) :' in ROBBINS, for specific $k$ and $n$.

Proof: Recall that $R=\pi(u)$. Since $\widetilde{P}_{R}$ is a $\operatorname{POLRAT}\left(x_{R} ; x_{\pi(1)}, \ldots, \widehat{x}_{R}, \ldots, x_{k}\right)$, we have that

$$
\begin{gathered}
\operatorname{Res}_{x_{\pi(u+1)}, \ldots, x_{\pi(k)}}\left[\frac{\widetilde{P}_{R}}{x_{R}^{n+k-R} \bar{x}_{R}^{n+k+1}}\right]=\operatorname{Res}_{x_{\pi(u+1)}, \ldots, x_{\pi(k)}}\left[\frac{\operatorname{POLRAT}\left(x_{R} ; x_{\pi(1)}, \ldots, \widehat{x}_{R}, x_{\pi(u+1)}, \ldots, x_{\pi(k)}\right)}{x_{R}^{n+k-R} \bar{x}_{R}^{n+k+1}}\right] \\
=\frac{\operatorname{POLRAT}\left(x_{R} ; x_{\pi(1)}, \ldots, x_{\pi(u-1)}\right)}{x_{R}^{n+k-R} \bar{x}_{R}^{n+k+1}}=\frac{x_{R}^{R+1} \cdot \operatorname{POLRAT}\left(x_{R} ; x_{\pi(1)}, \ldots, x_{\pi(u-1)}\right)}{x_{R}^{n+k+1} \bar{x}_{R}^{n+k+1}} .
\end{gathered}
$$

Since the degree of $x_{R}^{R+1} \operatorname{POLRAT}\left(x_{R} ; \ldots\right)$, in $x_{R}$, is $\leq(R+1)+k \leq 2(n+k)$, it follows from crucial fact $\aleph_{5}^{\prime}$ that

$$
\operatorname{Res}_{x_{R}}\left[\frac{\operatorname{POLRAT}\left(x_{R} ; \ldots\right)}{x_{R}^{n+k-R} \bar{x}_{R}^{n+k+1}}\right]=\operatorname{Res}_{x_{R}}\left[\frac{\operatorname{POLRAT}\left(x_{R} ; \ldots\right)}{x_{R}^{n+k-R} \bar{x}_{R}^{n+k+1}}\left(x_{R} \rightarrow \bar{x}_{R}\right)\right] .
$$

Applying $\operatorname{Res}_{x_{\pi(1)}, \ldots, x_{\pi(u-1)}}$ to both sides of the above equation establishes that $\frac{\widetilde{P}_{R}}{x_{R}^{n+k-R} \bar{x}_{R}^{n+k+1}}$ indeed has property (Hadas), so that (Hadas 1 ) holds. This completes the proof of subsubsublemma 1.3.1.3. 
Subsubsublemma 1.3.1.4: Let $\widetilde{B}_{i}$ be as in the statement of 1.3.1.2, i.e. given by $(\widetilde{B} i)$. Let $\pi$ be a permutation, $R$ an integer in $[1, k] \backslash\{i\}$, and $u:=\pi^{-1}(R)$. Then:

$$
\operatorname{Res}_{x_{\pi(1)}, \ldots, x_{\pi(k)}}\left[\frac{\widetilde{B}_{i}}{1-z_{i} x_{R}}\right]=\operatorname{Res}_{x_{\pi(1)}, \ldots, x_{\pi(k)}}\left[\frac{\widetilde{B}_{i}}{1-z_{i} x_{R}}\left(x_{R} \rightarrow \bar{x}_{R}\right)\right] .
$$

[ Type 'S1314 $(\mathrm{k}, \mathrm{n})$ :' in ROBBINS, for specific $\mathrm{k}$ and n.]

\section{Proof:}

Case I: $i \in\{\pi(u+1), \ldots, \pi(k)\}$.

Let $v:=\pi^{-1}(i)$, so that $i=\pi(v)$, and $v>u$. We have that:

$$
\frac{\widetilde{B}_{i}}{1-z_{i} x_{R}}=\frac{\operatorname{POLRAT}\left(x_{i} ; x_{\pi(1)}, \ldots, \widehat{x}_{R}, \ldots, x_{\pi(v-1)}, x_{\pi(v+1)}, \ldots, x_{\pi(k)}\right)}{x_{R}^{n+k-R} \bar{x}_{R}^{n+k+1} z_{i}^{n+2 k-i+1} \bar{z}_{i}^{n+k+1}\left(1-z_{i} x_{R}\right)} .
$$

Applying $\operatorname{Res}_{x_{\pi(v+1)}, \ldots, x_{\pi(k)}}$ to it, gets rid of the dependence on $\left(x_{\pi(v+1)}, \ldots, x_{\pi(k)}\right)$ :

$$
\operatorname{Res}_{x_{\pi(v+1)}, \ldots, x_{\pi(k)}}\left[\frac{\widetilde{B}_{i}}{1-z_{i} x_{R}}\right]=\frac{\operatorname{POLRAT}\left(x_{i} ; x_{\pi(1)}, \ldots, \widehat{x}_{R}, \ldots, x_{\pi(v-1)}\right)}{x_{R}^{n+k-R} \bar{x}_{R}^{n+k+1} z_{i}^{n+2 k-i+1} \bar{z}_{i}^{n+k+1}\left(1-z_{i} x_{R}\right)} .
$$

Applying $\operatorname{Res}_{x_{i}}$ to the above gives:

$$
\begin{aligned}
& \operatorname{Res}_{x_{i}, x_{\pi(v+1)}, \ldots, x_{\pi(k)}}\left[\frac{\widetilde{B}_{i}}{1-z_{i} x_{R}}\right]=\operatorname{Res}_{x_{i}}\left[\frac{\operatorname{POLRAT}\left(x_{i} ; x_{\pi(1)}, \ldots, \widehat{x}_{R}, \ldots, x_{\pi(v-1)}\right)}{x_{R}^{n+k-R} \bar{x}_{R}^{n+k+1} z_{i}^{n+2 k-i+1} \bar{z}_{i}^{n+k+1}\left(1-z_{i} x_{R}\right)}\right] \\
& =\frac{1}{x_{R}^{n+k-R} \bar{x}_{R}^{n+k+1}} \cdot \operatorname{Res}_{x_{i}}\left[\frac{\operatorname{POLRAT}\left(x_{i} ; x_{\pi(1)}, \ldots, \widehat{x}_{R}, \ldots, x_{\pi(v-1)}\right)}{z_{i}^{n+2 k-i+1} \bar{z}_{i}^{n+k+1}\left(1-z_{i} x_{R}\right)}\right] .
\end{aligned}
$$

We now distinguish two subcases:

Subcase Ia: $\varepsilon_{i}=+1$, that is: $z_{i}=x_{i}$.

In this subcase, the right side of (Doron) equals:

$$
\frac{1}{x_{R}^{n+k-R} \bar{x}_{R}^{n+k+1}} \cdot \operatorname{Coeff} f_{x_{i}^{n+2 k-i}}\left[\frac{\operatorname{POLRAT}\left(x_{i} ; x_{\pi(1)}, \ldots, \widehat{x}_{R}, \ldots, x_{\pi(v-1)}\right)}{\bar{x}_{i}^{n+k+1}\left(1-x_{i} x_{R}\right)}\right] .
$$

Expanding everything involving $x_{i}$ as a power series in $x_{i}$ :

$$
\begin{gathered}
\operatorname{POLRAT}\left(x_{i} ; x_{\pi(1)}, \ldots, \widehat{x}_{R}, \ldots, x_{\pi(v-1)}\right)=\sum_{t=0}^{\text {degree }} R A T_{t}\left(x_{\pi(1)}, \ldots, \widehat{x}_{R}, \ldots, x_{\pi(v-1)}\right) x_{i}^{t}, \\
\frac{1}{\bar{x}_{i}^{n+k+1}}=\sum_{t=0}^{\infty} N U M B E R_{t} \cdot x_{i}^{t},
\end{gathered}
$$


and, most importantly:

$$
\left(1-x_{i} x_{R}\right)^{-1}=\sum_{t=0}^{\infty} x_{R}^{t} x_{i}^{t}
$$

Multiplying out, and collecting the coefficient of $x_{i}^{n+2 k-i}$, gives that Coef $f_{x_{i}^{n+2 k-i}}$ in (Gil) is a certain polynomial in $x_{R}$, of degree $\leq n+2 k-i$, with coefficients that are rational functions of $\left(x_{\pi(1)}, \ldots, \widehat{x}_{R}, \ldots, x_{\pi(v-1)}\right)$. In other words it is a $\operatorname{POLRAT}\left(x_{R} ; x_{\pi(1)}, \ldots, x_{\pi(v-1)}\right)$ with $\operatorname{deg}_{x_{R}} \leq$ $n+2 k-i$. Hence,

$\operatorname{Res}_{x_{R}, x_{\pi(u+1)}, \ldots, x_{\pi(k)}}\left[\frac{\widetilde{B}_{i}}{1-x_{i} x_{R}}\right]=\operatorname{Res}_{x_{R}, x_{\pi(u+1)}, \ldots, x_{\pi(v-1)}}\left[\frac{\operatorname{POLRAT}\left(x_{R} ; x_{\pi(1)}, \ldots, \widehat{x}_{R}, \ldots, x_{\pi(v-1)}\right)}{x_{R}^{n+k-R} \bar{x}_{R}^{n+k+1}}\right]$

where the POLRAT in $\left(G i l^{\prime}\right)$ has degree $\leq n+2 k-i$ in $x_{R}$. Applying $\operatorname{Res}_{x_{\pi(u+1)}, \ldots, x_{\pi(v-1)}}$ to the residuand of $\left(G i l^{\prime}\right)$ gets rid of the dependence on $\left(x_{\pi(u+1)}, \ldots, x_{\pi(v-1)}\right)$, so we have:

$$
\operatorname{Res}_{x_{R}, x_{\pi(u+1)}, \ldots, x_{\pi(k)}}\left[\frac{\widetilde{B}_{i}}{1-x_{i} x_{R}}\right]=\operatorname{Res}_{x_{R}}\left[\frac{\operatorname{POLRAT}\left(x_{R} ; x_{\pi(1)}, \ldots, x_{\pi(u-1)}\right)}{x_{R}^{n+k-R} \bar{x}_{R}^{n+k+1}}\right] \quad, \quad\left(G i l^{\prime \prime}\right)
$$

where POLRAT $\left(x_{R} ; x_{\pi(1)}, \ldots, x_{\pi(u-1)}\right)$ has degree $\leq n+2 k-i$ in $x_{R}$. But, the right of $\left(G i l^{\prime \prime}\right)$ can be rewritten as:

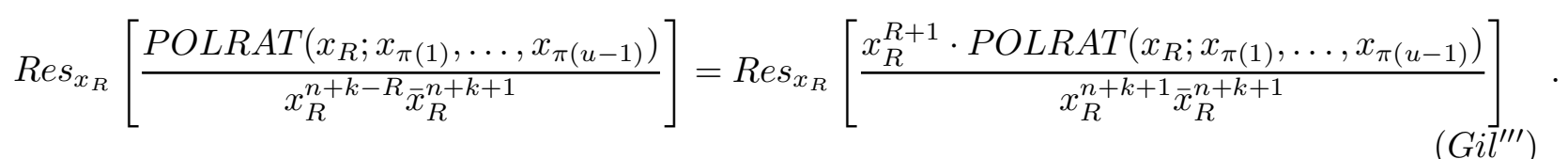

By crucial fact $\aleph_{5}^{\prime}$, since $(R+1)+(n+2 k-i) \leq 2(n+k)$ (recall that always $n \geq k \geq 1, R \leq k$, and $i \geq 1)$, we can perform $x_{R} \rightarrow \bar{x}_{R}$ in the residuand of $\left(G i l^{\prime \prime \prime}\right)$, so

$$
\operatorname{Res}_{x_{R}, x_{\pi(u+1)}, \ldots, x_{\pi(k)}}\left[\frac{\widetilde{B}_{i}}{1-x_{i} x_{R}}\right]=\operatorname{Res}_{x_{R}, x_{\pi(u+1)}, \ldots, x_{\pi(k)}}\left[\frac{\widetilde{B}_{i}}{1-x_{i} x_{R}}\left(x_{R} \rightarrow \bar{x}_{R}\right)\right] .
$$

Applying $\operatorname{Res}_{x_{\pi(1)}, \ldots, x_{\pi(u-1)}}$ to both sides of the above equation finishes up subcase Ia of the proof of $(s u b)^{3}$ lemma 1.3.1.4.

Subcase Ib: $\varepsilon_{i}=-1$, that is: $z_{i}=\bar{x}_{i}$.

In this subcase, the right side of (Doron) equals:

$$
\frac{1}{x_{R}^{n+k-R} \bar{x}_{R}^{n+k+1}} \cdot \operatorname{Coeff} x_{x_{i}^{n+k}}\left[\frac{\operatorname{POLRAT}\left(x_{i} ; x_{\pi(1)}, \ldots, \widehat{x}_{R}, \ldots, x_{\pi(v-1)}\right)}{\bar{x}_{i}^{n+2 k-i+1}\left(1-\bar{x}_{i} x_{R}\right)}\right] .
$$

Expanding everything involving $x_{i}$ as a power series in $x_{i}$ :

$$
\operatorname{POLRAT}\left(x_{i} ; x_{\pi(1)}, \ldots, \widehat{x}_{R}, \ldots, x_{\pi(v-1)}\right)=\sum_{t=0}^{\text {degree }} R A T_{t}\left(x_{\pi(1)}, \ldots, \widehat{x}_{R}, \ldots, x_{\pi(v-1)}\right) x_{i}^{t}
$$




$$
\frac{1}{\bar{x}_{i}^{n+2 k-i+1}}=\sum_{t=0}^{\infty} N U M B E R_{t} \cdot x_{i}^{t}
$$

and, most importantly:

$$
\left(1-\bar{x}_{i} x_{R}\right)^{-1}=\left(1-x_{R}+x_{i} x_{R}\right)^{-1}=\left(\bar{x}_{R}+x_{i} x_{R}\right)^{-1}=\sum_{t=0}^{\infty}(-1)^{t} \frac{x_{R}^{t}}{\bar{x}_{R}^{t+1}} x_{i}^{t}
$$

multiplying out, and collecting the coefficient of $x_{i}^{n+k}$, gives that $\operatorname{Coe} f f_{x_{i}^{n+k}}$ in $(G i l)$ is a certain sum of terms of the form

$$
R A T_{t}\left(x_{\pi(1)}, \ldots, \widehat{x}_{R}, \ldots, x_{\pi(v-1)}\right) \frac{x_{R}^{t}}{\bar{x}_{R}^{t+1}} \quad,(t \geq 0)
$$

Hence,

$$
\operatorname{Res}_{x_{R}, x_{\pi(u+1)}, \ldots, x_{\pi(k)}}\left[\frac{\widetilde{B}_{i}}{1-\bar{x}_{i} x_{R}}\right]
$$

is a sum of terms of the forms

$$
\operatorname{Res}_{x_{R}, x_{\pi(u+1)}, \ldots, x_{\pi(v-1)}}\left[\frac{x_{R}^{t} \cdot R A T_{t}\left(x_{\pi(1)}, \ldots, \widehat{x}_{R}, \ldots, x_{\pi(v-1)}\right)}{x_{R}^{n+k-R} \bar{x}_{R}^{n+k+t+2}}\right],(t \geq 0) .
$$

Applying $\operatorname{Res}_{x_{\pi(u+1)}, \ldots, x_{\pi(v-1)}}$ to the residuand of $\left(G i l^{\prime}\right)$ gets rid of the dependence on $\left(x_{\pi(u+1)}, \ldots, x_{\pi(v-1)}\right)$, so we have that

$$
\operatorname{Res}_{x_{R}, x_{\pi(u+1)}, \ldots, x_{\pi(k)}}\left[\frac{\widetilde{B}_{i}}{1-\bar{x}_{i} x_{R}}\right]
$$

is a sum of terms of the form

$$
\operatorname{Res}_{x_{R}}\left[\frac{x_{R}^{t} R A T_{t}\left(x_{\pi(1)}, \ldots, x_{\pi(u-1)}\right)}{x_{R}^{n+k-R} \bar{x}_{R}^{n+k+t+2}}\right],(t \geq 0)
$$

But, each such term can be written as

$$
R A T_{t}\left(x_{\pi(1)}, \ldots, x_{\pi(u-1)}\right) \operatorname{Res}_{x_{R}}\left[\frac{x_{R}^{R+2 t+2}}{x_{R}^{n+k+t+2} \bar{x}_{R}^{n+k+t+2}}\right] \quad, \quad(t \geq 0) \quad .
$$

By crucial fact $\aleph_{5}^{\prime}$, since $R+2 t+2 \leq 2(n+k+t+1)$, we can perform $x_{R} \rightarrow \bar{x}_{R}$ in the residuand of $\left(G i l^{\prime \prime \prime}\right)$, so

$$
\operatorname{Res}_{x_{R}, x_{\pi(u+1)}, \ldots, x_{\pi(k)}}\left[\frac{\widetilde{B}_{i}}{1-\bar{x}_{i} x_{R}}\right]=\operatorname{Res}_{x_{R}, x_{\pi(u+1)}, \ldots, x_{\pi(k)}}\left[\frac{\widetilde{B}_{i}}{1-\bar{x}_{i} x_{R}}\left(x_{R} \rightarrow \bar{x}_{R}\right)\right] .
$$

Applying $R e s_{x_{\pi(1)}, \ldots, x_{\pi(u-1)}}$ to both sides of the above equation finishes up subcase Ib of the proof of subsubsublemma 1.3.1.4.

Case II: $i \in\{\pi(1), \ldots, \pi(u-1)\}$. 
The proof is identical to Case II of $(s u b)^{4}$ lemma 1.4.1.4 proved in act IV (except that the form of $\widetilde{B}_{i}$ is slightly more complicated there, but the argument that reduces it to Case Ia goes verbatim). This completes the proof of $(s u b)^{3}$ lemma 1.3.1.4.

Going back to the proof of $(s u b)^{2}$ lemma 1.3.1, we know by the two $(s u b)^{3}$ lemmas $(1.3 .1 .3-4)$ that each part of (Celia), in the statement of $(s u b)^{3}$ lemma 1.3.1.2, has property (Hadas), and by the additivity of this property, so does the whole $f_{n, k}\left(\varepsilon_{1}\left(x_{1}\right), \ldots, x_{R}, \ldots, \varepsilon_{k}\left(x_{k}\right)\right)$. But this is precisely what $(s u b)^{2}$ lemma 1.3.1 is saying. This completes the proof of $(s u b)^{2}$ lemma 1.3.1.

As we noted at the very beginning of the proof of sublemma 1.3, subsublemma 1.3.1, read from right to left, can be used repeatedly to 'unbar' $x_{i}$ 's, until we get that

$$
\operatorname{Res}_{x_{\pi(1)}, \ldots, x_{\pi(k)}}\left[f_{n, k}\left(\varepsilon_{1}\left(x_{1}\right), \ldots, \varepsilon_{k}\left(x_{k}\right)\right)\right]=\operatorname{Res}_{x_{\pi(1)}, \ldots, x_{\pi(k)}}\left[f_{n, k}\left(x_{1}, \ldots, x_{k}\right)\right] .
$$

Since $f_{n, k}\left(x_{1}, \ldots, x_{k}\right)$ has a genuine Laurent expansion, crucial fact $\aleph_{4}^{\prime}$ assures us that we can unscramble the order of residue-taking on the right side of (Ktsat):

$$
\operatorname{Res}_{x_{\pi(1)}, \ldots, x_{\pi(k)}}\left[f_{n, k}\left(x_{1}, \ldots, x_{k}\right)\right]=\operatorname{Res}_{x_{1}, \ldots, x_{k}}\left[f_{n, k}\left(x_{1}, \ldots, x_{k}\right)\right]
$$

Combining (Ktsat) with (Nimas) yields $\left(1.3^{\prime}\right)$, which we noted was equivalent to the statement of sublemma 1.3. This completes the proof of sublemma 1.3. 


\section{Act IV. (GogTotal') IS UNFAZED BY THE SIGNED PERMUTATIONS' ACTION}

... signed ...-(Daniel VI, 10)

Sublemma 1.4 : Let $F_{n, k}(x)=F_{n, k}\left(x_{1}, \ldots, x_{k}\right)$ be the residuand of $($ GogTotal $)$, that is,

$$
F_{n, k}\left(x_{1}, \ldots, x_{k}\right):=\frac{\Phi_{k}\left(x_{1}, \ldots, x_{k}\right)}{\prod_{i=1}^{k} x_{i}^{n+1} \bar{x}_{i}^{n+i+1} \prod_{1 \leq i<j \leq k}\left(1-x_{i} x_{j}\right)\left(1-\bar{x}_{i} x_{j}\right)} \quad,
$$

where $\Phi_{k}\left(x_{1}, \ldots, x_{k}\right)$ is the polynomial defined in $\left(G_{0} g_{1}\right)$. Let $g$ be any signed permutation in $W\left(\mathcal{B}_{k}\right)$, then

$$
\operatorname{Res}_{x_{1}, \ldots, x_{k}}\left[g F_{n, k}(x)\right]=\operatorname{Res}_{x_{1}, \ldots, x_{k}}\left[F_{n, k}(x)\right] \quad\left(=m_{k}(n)\right) .
$$

[ Type 'S14 $(\mathrm{k}, \mathrm{n})$ :' in ROBBINS, for specific $\mathrm{k}$ and $\mathrm{n}$.

Proof: As in the proof of 1.3 , by renaming the variables, the statement of sublemma 1.4 is equivalent to

Sublemma 1.4' : Let $F_{n, k}(x)=F_{n, k}\left(x_{1}, \ldots, x_{k}\right)$ be the residuand of (GogTotal'), reproduced just above. Let $\pi$ be any permutation of $\{1, \ldots, k\}$, and let $\varepsilon=\left(\varepsilon_{1}, \ldots, \varepsilon_{k}\right)$ be any sign-assignment. Then

$$
\operatorname{Res}_{x_{\pi(1)}, \ldots, x_{\pi(k)}}\left[F_{n, k}\left(\varepsilon_{1}\left(x_{1}\right), \ldots, \varepsilon_{k}\left(x_{k}\right)\right)\right]=\operatorname{Res}_{x_{1}, \ldots, x_{k}}\left[F_{n, k}\left(x_{1}, \ldots, x_{k}\right)\right]
$$

The proof will be by induction on the number of $\varepsilon_{i}$ 's that are equal to -1 . If there are no -1 's in $\varepsilon$, then $1.4^{\prime}$ follows immediately from crucial fact $\aleph_{4}$, since then the residuand $F_{n, k}\left(x_{1}, \ldots, x_{k}\right)$ has a Laurent series. Otherwise, let $u$ be the smallest integer $i(1 \leq i \leq k)$ such that $\varepsilon_{\pi(i)}=-1$. The inductive step is provided by the following subsublemma, that asserts that it is permissible to change $\varepsilon_{\pi(u)}$ from -1 to +1 inside the residuand of the iterated residue on the left side of $\left(1.4^{\prime}\right)$ without affecting its value.

Subsublemma 1.4.1: Let $F_{n, k}\left(x_{1}, \ldots, x_{k}\right)$ be the residuand of (GogTotal'), let $\pi$ be any permutation of $\{1, \ldots, k\}$, and let $\varepsilon=\left(\varepsilon_{1}, \ldots, \varepsilon_{k}\right)$ be any sign-assignment with at least one $\varepsilon_{i}$ equal to -1 . Let $u$ be the smallest $i(1 \leq i \leq k)$ such that $\varepsilon_{\pi(i)}=-1$. (i.e., we have $\varepsilon_{\pi(1)}=\ldots=\varepsilon_{\pi(u-1)}=+1$, and $\left.\varepsilon_{\pi(u)}=-1\right)$. Then:

$$
\operatorname{Res}_{x_{\pi(1)}, \ldots, x_{\pi(k)}}\left[F_{n, k}\left(\varepsilon_{1}\left(x_{1}\right), \ldots, x_{\pi(u)}, \ldots, \varepsilon_{k}\left(x_{k}\right)\right)\right]=\operatorname{Res}_{x_{\pi(1)}, \ldots, x_{\pi(k)}}\left[F_{n, k}\left(\varepsilon_{1}\left(x_{1}\right), \ldots, \bar{x}_{\pi(u)}, \ldots, \varepsilon_{k}\left(x_{k}\right)\right)\right]
$$

[ Type 'S141 $(\mathrm{k}, \mathrm{n})$ :' in ROBBINS, for specific $\mathrm{k}$ and $\mathrm{n}$.

Proof: Let's abbreviate $R:=\pi(u)$, and $z_{i}:=\varepsilon_{i}\left(x_{i}\right)(1 \leq i \leq k, i \neq R)$. Recall that this means that $z_{i}=x_{i}$ if $\varepsilon_{i}=+1$, and $z_{i}=\bar{x}_{i}=1-x_{i}$ if $\varepsilon_{i}=-1$. Also put $z_{R}=x_{R}$. By the $W\left(\mathcal{B}_{k}\right)$-antisymmetry of $\Phi_{k}(x)$, we have

$$
F_{n, k}\left(\varepsilon_{1}\left(x_{1}\right), \ldots, x_{R}, \ldots, \varepsilon_{k}\left(x_{k}\right)\right)=\frac{ \pm \Phi_{k}\left(x_{1}, \ldots, x_{k}\right)}{x_{R}^{n+1} \bar{x}_{R}^{n+R+1} \prod_{\substack{j=1 \\ j \neq R}}^{k} z_{j}^{n+1} \bar{z}_{j}^{n+j+1} \prod_{1 \leq i<j \leq k}\left(1-z_{i} z_{j}\right)\left(1-\bar{z}_{i} z_{j}\right)}
$$


THE Electronic Journal of COMbinatorics 3 (2) (1996), \#R13

$$
\begin{aligned}
& =\frac{1}{x_{R}^{n+1} \bar{x}_{R}^{n+R+1}} \prod_{\substack{j=1 \\
j \neq R}}^{k} \frac{1}{z_{j}^{n+1} \bar{z}_{j}^{n+j+1}} \prod_{\substack{1 \leq r<s \leq k \\
r, s \neq R}} \frac{1}{\left(1-z_{r} z_{s}\right)\left(1-\bar{z}_{r} z_{s}\right)} . \\
& \left\{\frac{ \pm \Phi_{k}\left(x_{1}, \ldots, x_{k}\right)}{\prod_{\substack{i=1 \\
i \neq R}}^{k}\left(1-z_{i} x_{R}\right) \prod_{i=1}^{R-1}\left(1-\bar{z}_{i} x_{R}\right) \prod_{i=R+1}^{k}\left(1-z_{i} \bar{x}_{R}\right)}\right\} .
\end{aligned}
$$

The next step is to perform a partial-fraction decomposition, with respect to the variable $x_{R}$, of the fraction inside (Jane)'s braces.

Subsubsublemma 1.4.1.1:

$$
\begin{aligned}
& \frac{ \pm \Phi_{k}\left(x_{1}, \ldots, x_{k}\right)}{\prod_{\substack{i=1 \\
i \neq R}}^{k}\left(1-z_{i} x_{R}\right) \prod_{i=1}^{R-1}\left(1-\bar{z}_{i} x_{R}\right) \prod_{i=R+1}^{k}\left(1-z_{i} \bar{x}_{R}\right)} \\
= & P_{R}+\sum_{\substack{i=1 \\
i \neq R}}^{k} \frac{A_{i}}{1-z_{i} x_{R}}+\sum_{i=1}^{R-1} \frac{B_{i}}{1-\bar{z}_{i} x_{R}}+\sum_{i=R+1}^{k} \frac{B_{i}}{1-z_{i} \bar{x}_{R}}
\end{aligned}
$$

where $P_{R}$ is a polynomial of degree $\leq 2 k-1$ in $x_{R}$, with coefficients that are rational functions of the other variables $\left(x_{1}, \ldots, x_{R-1}, x_{R+1}, \ldots, x_{k}\right)$, and the $A_{i}$ 's and $B_{i}$ 's are as follows.

When $1 \leq i<R$,

$$
\begin{gathered}
A_{i}=\frac{ \pm\left(z_{i}-2\right)\left(1-z_{i}^{2}\right)\left(1-z_{i} \bar{z}_{i}\right) \Phi_{k-2}\left(x_{1}, \ldots, \widehat{x}_{i}, \ldots, \widehat{x}_{R}, \ldots, x_{k}\right)}{z_{i}^{k}} \\
\left\{\prod_{\substack{j=1 \\
j \neq i, R}}^{k}\left(1-z_{i} z_{j}\right)\left(1-\bar{z}_{i} z_{j}\right)\left(1-z_{i} \bar{z}_{j}\right)\right\} \prod_{j=R+1}^{k}\left(z_{j}+z_{i}-1\right) \prod_{\substack{j=1 \\
j \neq i}}^{R-1}\left(1-\bar{z}_{i} \bar{z}_{j}\right) .
\end{gathered}
$$

When $R<i \leq k$, we have:

$$
\begin{gathered}
A_{i}=\frac{ \pm\left(1-2 z_{i}\right)\left(1-z_{i}^{2}\right)\left(1-z_{i} \bar{z}_{i}\right) \Phi_{k-2}\left(x_{1}, \ldots, \widehat{x}_{R}, \ldots, \widehat{x}_{i}, \ldots, x_{k}\right)}{z_{i}^{k+1}} . \\
\left\{\prod_{\substack{j=1 \\
j \neq i, R}}^{k}\left(1-z_{i} z_{j}\right)\left(1-\bar{z}_{i} z_{j}\right)\left(1-z_{i} \bar{z}_{j}\right)\right\} \prod_{\substack{j=R+1 \\
j \neq i}}^{k}\left(z_{j}+z_{i}-1\right) \prod_{j=1}^{R-1}\left(1-\bar{z}_{i} \bar{z}_{j}\right) .
\end{gathered}
$$

As for the $B_{i}$, when $1 \leq i<R$, we have

$$
\begin{gathered}
B_{i}=\frac{ \pm\left(\bar{z}_{i}-2\right)\left(1-\bar{z}_{i}^{2}\right)\left(1-z_{i} \bar{z}_{i}\right) \Phi_{k-2}\left(x_{1}, \ldots, \widehat{x}_{i}, \ldots, \widehat{x}_{R}, \ldots, x_{k}\right)}{\bar{z}_{i}^{k}} \\
\left\{\prod_{\substack{j=1 \\
j \neq i, R}}^{k}\left(1-z_{i} z_{j}\right)\left(1-\bar{z}_{i} z_{j}\right)\left(1-\bar{z}_{i} \bar{z}_{j}\right)\right\} \prod_{j=R+1}^{k}\left(z_{j}-z_{i}\right) \prod_{\substack{j=1 \\
j \neq i}}^{R-1}\left(1-z_{i} \bar{z}_{j}\right) .
\end{gathered}
$$


Finally, when $R<i \leq k$, we have:

$$
\begin{gathered}
B_{i}=\frac{ \pm\left(1-2 z_{i}\right)\left(1-z_{i}^{2}\right)\left(1-z_{i} \bar{z}_{i}\right) \Phi_{k-2}\left(x_{1}, \ldots, \widehat{x}_{R}, \ldots, \widehat{x}_{i}, \ldots, x_{k}\right)}{z_{i}^{k+1}} \\
\left\{\prod_{\substack{j=1 \\
j \neq i, R}}^{k}\left(1-z_{i} z_{j}\right)\left(1-z_{i} \bar{z}_{j}\right)\left(z_{i}+z_{j}-1\right)\right\} \prod_{\substack{j=R+1 \\
j \neq i}}^{k}\left(1-\bar{z}_{i} z_{j}\right) \prod_{j=1}^{R-1}\left(z_{i}-z_{j}\right) .
\end{gathered}
$$

[ Type 'S1411 $(k, R)$ :' in ROBBINS, for specific $k$ and R.]

Proof: By Freshman Calculus (or rather the algebraic part of it), we know that the left side of (Tamar), when viewed as a rational function of $x_{R}$, can be written as on its right side, for some polynomial $P_{R}$, and constants (in the present context, i.e. quantities not involving $x_{R}$ ) $A_{i}, B_{i}$. The degree of $\Phi_{k}$, in $x_{R}$ (or for that matter, in any variable), from its definition in $\left(G_{0} g_{1}\right)$, is $\leq 4 k-3$, and the degree of the denominator of the left side of (Tamar) is exactly $2(k-1)$. Hence the degree of $P_{R}$, in $x_{R}$, is $\leq(4 k-3)-(2 k-2)=2 k-1$. The coefficients of $P_{R}$ are certain rational functions of the remaining variables whose exact form does not interest us. The exact forms of $A_{i}$ and $B_{i}$ should interest us, since they are used heavily in the proof. So let's brace ourselves and embark on partial-fractioning.

Multiplying both sides of (Tamar) by the denominator of its left side yields the equation:

$$
\begin{aligned}
& \pm \Phi_{k}\left(x_{1}, \ldots, x_{k}\right)=\left\{\prod_{\substack{j=1 \\
j \neq R}}^{k}\left(1-z_{j} x_{R}\right) \prod_{j=1}^{R-1}\left(1-\bar{z}_{j} x_{R}\right) \prod_{j=R+1}^{k}\left(1-z_{j} \bar{x}_{R}\right)\right\} P_{R} \\
& +\sum_{\substack{i=1 \\
i \neq R}}^{k}\left\{\prod_{\substack{j=1 \\
j \neq R, i}}^{k}\left(1-z_{j} x_{R}\right) \prod_{j=1}^{R-1}\left(1-\bar{z}_{j} x_{R}\right) \prod_{j=R+1}^{k}\left(1-z_{j} \bar{x}_{R}\right)\right\} A_{i} \\
& +\sum_{i=1}^{R-1}\left\{\prod_{\substack{j=1 \\
j \neq R}}^{k}\left(1-z_{j} x_{R}\right) \prod_{\substack{j=1 \\
j \neq i}}^{R-1}\left(1-\bar{z}_{j} x_{R}\right) \prod_{j=R+1}^{k}\left(1-z_{j} \bar{x}_{R}\right)\right\} B_{i} \\
& +\sum_{i=R+1}^{k}\left\{\prod_{\substack{j=1 \\
j \neq R}}^{k}\left(1-z_{j} x_{R}\right) \prod_{j=1}^{R-1}\left(1-\bar{z}_{j} x_{R}\right) \prod_{\substack{j=R+1 \\
j \neq i}}^{k}\left(1-z_{j} \bar{x}_{R}\right)\right\} B_{i} \cdot
\end{aligned}
$$

To decipher $A_{i}(1 \leq i \leq k, i \neq R)$, we plug in $x_{R}=1 / z_{i}$ on both sides of (Tamar') to get:

$$
\begin{gathered}
A_{i}=\frac{ \pm\left.\Phi_{k}\left(x_{1}, \ldots, x_{k}\right)\right|_{x_{R}=1 / z_{i}}}{\left\{\prod_{\substack{j=1 \\
j \neq R, i}}^{k}\left(1-z_{j} / z_{i}\right) \prod_{j=1}^{R-1}\left(1-\bar{z}_{j} / z_{i}\right) \prod_{j=R+1}^{k}\left(1-z_{j}\left(1-1 / z_{i}\right)\right)\right\}} \\
=\frac{ \pm\left. z_{i}^{2 k-3} \Phi_{k}\left(x_{1}, \ldots, x_{k}\right)\right|_{x_{R}=1 / z_{i}}}{\left\{\prod_{\substack{j=1 \\
j \neq R, i}}^{k}\left(z_{i}-z_{j}\right) \prod_{j=1}^{R-1}\left(z_{i}-\bar{z}_{j}\right) \prod_{j=R+1}^{k}\left(1-\bar{z}_{i} \bar{z}_{j}\right)\right\}} .
\end{gathered}
$$


To decipher $B_{i}(1 \leq i<R)$, we plug in $x_{R}=1 / \bar{z}_{i}$ on both sides of $\left(\right.$ Tamar $\left.^{\prime}\right)$ to get:

$$
\begin{gathered}
B_{i}=\frac{ \pm\left.\Phi_{k}\left(x_{1}, \ldots, x_{k}\right)\right|_{x_{R}=1 / \bar{z}_{i}}}{\left\{\prod_{\substack{j=1 \\
j \neq R}}^{k}\left(1-z_{j} / \bar{z}_{i}\right) \prod_{\substack{j=1 \\
j \neq i}}^{R-1}\left(1-\bar{z}_{j} / \bar{z}_{i}\right) \prod_{j=R+1}^{k}\left(1-z_{j}\left(1-1 / \bar{z}_{i}\right)\right)\right\}} \\
=\frac{ \pm\left.\bar{z}_{i}^{2 k-3} \Phi_{k}\left(x_{1}, \ldots, x_{k}\right)\right|_{x_{R}=1 / \bar{z}_{i}}}{\left\{\prod_{\substack{j=1 \\
j \neq R}}^{k}\left(\bar{z}_{i}-z_{j}\right) \prod_{\substack{k=1 \\
j \neq i}}^{R-1}\left(\bar{z}_{i}-\bar{z}_{j}\right) \prod_{j=R+1}^{k}\left(1-z_{i} \bar{z}_{j}\right)\right\}} \quad(1 \leq i<R) .
\end{gathered}
$$

To decipher $B_{i}(R<i \leq k)$, we plug in $\bar{x}_{R}=1 / z_{i}$, i.e. $x_{R}=1-1 / z_{i}=-\bar{z}_{i} / z_{i}$, on both sides of (Tamar') to get:

$$
\begin{gathered}
B_{i}=\frac{ \pm\left.\Phi_{k}\left(x_{1}, \ldots, x_{k}\right)\right|_{\bar{x}_{R}=1 / z_{i}}}{\left\{\prod_{\substack{j=1 \\
j \neq R}}^{k}\left(1+z_{j} \bar{z}_{i} / z_{i}\right) \prod_{j=1}^{R-1}\left(1+\bar{z}_{j} \bar{z}_{i} / z_{i}\right) \prod_{\substack{k=R+1 \\
j \neq i}}^{k}\left(1-z_{j} / z_{i}\right)\right\}} \\
=\frac{ \pm\left. z_{i}^{2 k-3} \Phi_{k}\left(x_{1}, \ldots, x_{k}\right)\right|_{\bar{x}_{R}=1 / z_{i}}}{\left\{\prod_{\substack{j=1 \\
j \neq R}}^{k}\left(1-\bar{z}_{i} \bar{z}_{j}\right) \prod_{j=1}^{R-1}\left(1-\bar{z}_{i} z_{j}\right) \prod_{\substack{k=R+1 \\
j \neq i}}^{k}\left(z_{i}-z_{j}\right)\right\}} \quad(R<i \leq k) .
\end{gathered}
$$

We now need:

Subsubsubsublemma 1.4.1.1.1: For $1 \leq R \neq i \leq k$ :

$$
\begin{gathered}
\left.\Phi_{k}\left(x_{1}, x_{2}, \ldots, x_{k}\right)\right|_{x_{R}=1 / z_{i}}= \pm \frac{\left(z_{i}-2\right)\left(1-2 z_{i}\right)\left(1-z_{i}^{2}\right)\left(1-z_{i} \bar{z}_{i}\right) \cdot \Phi_{k-2}\left(x_{1}, \ldots, \widehat{x}_{i}, \ldots, \widehat{x}_{R}, \ldots, x_{k}\right)}{z_{i}^{3 k-3}} . \\
\prod_{\substack{j=1 \\
j \neq i, R}}^{k}\left\{\left(1-z_{i} z_{j}\right)\left(1-\bar{z}_{i} z_{j}\right)\left(1-z_{i} \bar{z}_{j}\right)\left(1-\bar{z}_{i} \bar{z}_{j}\right)\left(z_{j}-z_{i}\right)\left(z_{j}+z_{i}-1\right)\right\}
\end{gathered}
$$

[ Type 'S14111(k,R,i) :' in ROBBINS, for specific $k, R$, and i.]

Proof: By the anti-symmetry of $\Phi_{k}$ with respect to the symmetric group, it suffices to consider $R=1$. By its anti-symmetry w.r.t. the sign-action, it suffices to consider the case $z_{i}=x_{i}$ (i.e. $\left.\varepsilon_{i}=+1\right)$. But this follows from multiplying the respective sides of the identities of $(s u b)^{4}$ lemma 1.3.1.1.1 (proved above) and subsublemma 1.5.2 (proved below). This completes the proof of $(s u b)^{4}$ lemma 1.4.1.1.1.

Substituting (1.4.1.1.1) into (PP1), (PP2), and (PP3), and cancelling out whenever possible, finishes up the proof of $(s u b)^{3}$ lemma 1.4.1.1. For $(P P 3)$ we must note that $\Phi_{k}$ remains the same, except for a sign change, when one replaces $x_{R}$ by $\bar{x}_{R}$, so we can also apply 1.4.1.1.1 with $x_{R}$ replaced by $\bar{x}_{R}$. This completes the proof of $(s u b)^{3}$ lemma 1.4.1.1.

For the rest of the proof of $(s u b)^{2}$ lemma 1.4.1, we need some new notation. Let $P O L$ stand for "some polynomial of $\left(x_{1}, \ldots, x_{k}\right)$ ", and let $R A T$ stand for "some rational function of $\left(x_{1}, \ldots, x_{k}\right)$ ". $\operatorname{POL}\left(\widehat{x}_{R}\right)$ stands for such a polynomial that does not involve $x_{R} \operatorname{POLRAT}\left(x_{R} ; x_{1}, \ldots, \widehat{x}_{R}, \ldots, x_{k}\right)$, or $\operatorname{POLRAT}\left(x_{R} ; \ldots\right)$ for short, stands for a polynomial in $x_{R}$ with coefficients that are rational 
functions of the rest of the variables. POLRAT $\left(x_{i} ; \widehat{x}_{R}\right)$ stands for a polynomial in $x_{i}$ with coefficients that are rational functions of the remaining variables and that do not involve $x_{R}$.

Inserting (Tamar) (in the statement of 1.4.1.1) into (Jane) is going to yield the following $(s u b)^{3}$ lemma:

Subsubsublemma 1.4.1.2:

$$
\begin{gathered}
F_{n, k}\left(\varepsilon_{1}\left(x_{1}\right), \ldots, x_{R}, \ldots, \varepsilon_{k}\left(x_{k}\right)\right) \\
=\frac{\widetilde{P}_{R}}{x_{R}^{n+1} \bar{x}_{R}^{n+R+1}}+\sum_{\substack{i=1 \\
i \neq R}}^{k} \frac{\widetilde{A}_{i}}{1-z_{i} x_{R}}+\sum_{i=1}^{R-1} \frac{\widetilde{B}_{i}}{1-\bar{z}_{i} x_{R}}+\sum_{i=R+1}^{k} \frac{\widetilde{B}_{i}}{1-z_{i} \bar{x}_{R}},
\end{gathered}
$$

where $\widetilde{P}_{R}$ is a polynomial of degree $\leq 2 k-1$ in $x_{R}$, with coefficients that are rational functions of the rest of the variables, and $\widetilde{A}_{i}$, and $\widetilde{B}_{i}$ can be written as follows.

For $1 \leq i<R$, we have

$$
\begin{gathered}
\widetilde{A}_{i}=\frac{\operatorname{POL}\left(\widehat{x}_{R}\right)}{x_{R}^{n+1} \bar{x}_{R}^{n+R+1} z_{i}^{n+k+1} \bar{z}_{i}^{n+i+1}} \cdot \prod_{\substack{j=1 \\
j \neq i, R}}^{k} \frac{1}{z_{j}^{n+1} \bar{z}_{j}^{n+j+1}} \cdot \prod_{\substack{1 \leq r<s \leq k \\
r, s \neq R, i}} \frac{1}{\left(1-z_{r} z_{s}\right)\left(1-\bar{z}_{r} z_{s}\right)} \\
=\frac{\operatorname{POLRAT}\left(x_{i} ; \widehat{x}_{R}\right)}{x_{R}^{n+1} \bar{x}_{R}^{n+R+1} z_{i}^{n+k+1} \bar{z}_{i}^{n+i+1}}
\end{gathered}
$$

For $R<i \leq k$, we have

$$
\begin{gathered}
\widetilde{A}_{i}=\frac{\operatorname{POL}\left(\widehat{x}_{R}\right)}{x_{R}^{n+1} \bar{x}_{R}^{n+R+1} z_{i}^{n+k+2} \bar{z}_{i}^{n+i+1}} \cdot \prod_{\substack{j=1 \\
j \neq i, R}}^{k} \frac{1}{z_{j}^{n+1} \bar{z}_{j}^{n+j+1}} \cdot \prod_{\substack{1 \leq r<s \leq k \\
r, s \neq R, i}} \frac{1}{\left(1-z_{r} z_{s}\right)\left(1-\bar{z}_{r} z_{s}\right)} \\
=\frac{\operatorname{POLRAT}\left(x_{i} ; \widehat{x}_{R}\right)}{x_{R}^{n+1} \bar{x}_{R}^{n+R+1} z_{i}^{n+k+2} \bar{z}_{i}^{n+i+1}}
\end{gathered}
$$

For $1 \leq i<R$, we have

$$
\begin{gathered}
\widetilde{B}_{i}=\frac{\operatorname{POL}\left(\widehat{x}_{R}\right)}{x_{R}^{n+1} \bar{x}_{R}^{n+R+1} z_{i}^{n+1} \bar{z}_{i}^{n+i+k+1}} \cdot \prod_{\substack{j=1 \\
j \neq i, R}}^{k} \frac{1}{z_{j}^{n+1} \bar{z}_{j}^{n+j+1}} \cdot \prod_{\substack{1 \leq r<s \leq k \\
r, s \neq R, i}} \frac{1}{\left(1-z_{r} z_{s}\right)\left(1-\bar{z}_{r} z_{s}\right)} \\
=\frac{\operatorname{POLRAT}\left(x_{i} ; \widehat{x}_{R}\right)}{x_{R}^{n+1} \bar{x}_{R}^{n+R+1} z_{i}^{n+1} \bar{z}_{i}^{n+i+k+1}} .
\end{gathered}
$$

Finally, for $R<i \leq k$, we have

$$
\widetilde{B}_{i}=\frac{\operatorname{POL}\left(\widehat{x}_{R}\right)}{x_{R}^{n+1} \bar{x}_{R}^{n+R+1} z_{i}^{n+k+2} \bar{z}_{i}^{n+i+1}} \cdot \prod_{\substack{j=1 \\ j \neq i, R}}^{k} \frac{1}{z_{j}^{n+1} \bar{z}_{j}^{n+j+1}} \cdot \prod_{\substack{1 \leq r<s \leq k \\ r, s \neq R, i}} \frac{1}{\left(1-z_{r} z_{s}\right)\left(1-\bar{z}_{r} z_{s}\right)}
$$


THE Electronic Journal of COMbinatorics 3 (2) (1996), \#R13

$$
=\frac{P O L R A T\left(x_{i} ; \widehat{x}_{R}\right)}{x_{R}^{n+1} \bar{x}_{R}^{n+R+1} z_{i}^{n+k+2} \bar{z}_{i}^{n+i+1}}
$$

Furthermore, all the POLRATS are of degree $\leq 2 k+1$ in $x_{i}$ (we actually only use this fact for the POLRAT of $(\widetilde{B} i 1))$.

[ Type 'S1412(k,n,eps, R) :' in ROBBINS, for specific k,n,eps, and R.]

Proof: Insert (Tamar) into (Jane), bringing the quantity in front of the sums inside, and for each $A_{i}$ and $B_{i}$, split the double product:

$$
\prod_{\substack{1 \leq r<s \leq k \\ r, s \neq R}} \frac{1}{\left(1-z_{r} z_{s}\right)\left(1-\bar{z}_{r} z_{s}\right)}=\prod_{\substack{1 \leq r<s \leq k \\ r, s \neq R, i}} \frac{1}{\left(1-z_{r} z_{s}\right)\left(1-\bar{z}_{r} z_{s}\right)} \cdot \prod_{\substack{j=1 \\ j \neq R}}^{i-1} \frac{1}{\left(1-z_{j} z_{i}\right)\left(1-\bar{z}_{j} z_{i}\right)} \prod_{\substack{j=i+1 \\ j \neq R}}^{k} \frac{1}{\left(1-z_{i} z_{j}\right)\left(1-\bar{z}_{i} z_{j}\right)}
$$

where the condition $j \neq R$ is vacuous in exactly one of the two last products according to whether $i<R$ or $i>R$. Finally perform all possible cancellations. This completes the proof of $(s u b)^{3}$ lemma 1.4.1.2.

We will say that a rational function $h=h\left(x_{1}, \ldots, x_{k}\right)$ has property (Hadas) if:

$$
\operatorname{Res}_{x_{\pi(1)}, \ldots, x_{\pi(k)}}[h]=\operatorname{Res}_{x_{\pi(1)}, \ldots, x_{\pi(k)}}\left[h\left(x_{R} \rightarrow \bar{x}_{R}\right)\right],
$$

where $h\left(x_{R} \rightarrow \bar{x}_{R}\right)$ is the rational function obtained from $h$ by replacing the variable $x_{R}$ by $\bar{x}_{R}$ $\left(=1-x_{R} \cdot\right)$

The statement of subsublemma 1.4.1 is equivalent to saying that

$$
F_{n, k}\left(\varepsilon_{1}\left(x_{1}\right), \ldots, x_{R}, \ldots, \varepsilon_{k}\left(x_{k}\right)\right)
$$

has property (Hadas). Property (Hadas) is obviously additive (since the action of taking residue, and performing $x_{R} \rightarrow \bar{x}_{R}$ are), so in order to complete the proof of 1.4 .1 (and hence of 1.4), it would suffice to show that each term in (Celia) has property (Hadas). This will be accomplished by the following series of subsubsublemmas $(1.4 .1 .3-6$.)

Subsubsublemma 1.4.1.3: Let $\widetilde{P}_{R}$ be as in the statement of 1.4 .1 .2 , i.e. a polynomial of degree $\leq 2 \underset{P_{R}}{ }-1$ in $x_{R}$ with coefficients that are rational functions of the remaining variables. Then $\frac{\widetilde{P}_{R}}{x_{R}^{n+1} \bar{x}_{R}^{n+R+1}}$ has property (Hadas):

$$
\operatorname{Res}_{x_{\pi(1)}, \ldots, x_{\pi(k)}}\left[\frac{\widetilde{P}_{R}}{x_{R}^{n+1} \bar{x}_{R}^{n+R+1}}\right]=\operatorname{Res}_{x_{\pi(1)}, \ldots, x_{\pi(k)}}\left[\frac{\widetilde{P}_{R}}{x_{R}^{n+1} \bar{x}_{R}^{n+R+1}}\left(x_{R} \rightarrow \bar{x}_{R}\right)\right] .
$$

[ Type 'S1413(k,n):' in ROBBINS, for specific $k$ and $n$.

Proof: Recall that $R=\pi(u)$. Since $\widetilde{P}_{R}$ is a $\operatorname{POLRAT}\left(x_{R} ; x_{1}, \ldots, \widehat{x}_{R}, \ldots, x_{k}\right)$, we have that

$$
\operatorname{Res}_{x_{\pi(u+1)}, \ldots, x_{\pi(k)}}\left[\frac{\widetilde{P}_{R}}{x_{R}^{n+1} \bar{x}_{R}^{n+R+1}}\right]=\operatorname{Res}_{x_{\pi(u+1)}, \ldots, x_{\pi(k)}}\left[\frac{\operatorname{POLRAT}\left(x_{R} ; x_{\pi(1)}, \ldots, \widehat{x}_{R}, x_{\pi(u+1)}, \ldots, x_{\pi(k)}\right)}{x_{R}^{n+1} \bar{x}_{R}^{n+R+1}}\right]
$$


THE Electronic Journal of COMbinatorics 3 (2) (1996), \#R13

$$
=\frac{\operatorname{POLRAT}\left(x_{R} ; x_{\pi(1)}, \ldots, x_{\pi(u-1)}\right)}{x_{R}^{n+1} \bar{x}_{R}^{n+R+1}}=\frac{x_{R}^{R} \cdot \operatorname{POLRAT}\left(x_{R} ; x_{\pi(1)}, \ldots, x_{\pi(u-1)}\right)}{x_{R}^{n+R+1} \bar{x}_{R}^{n+R+1}} .
$$

Since the degree of $x_{R}^{R} P O L R A T\left(x_{R} ; \ldots\right)$, in $x_{R}$, is $\leq R+(2 k-1) \leq 2(n+R)$, it follows from crucial fact $\aleph_{5}^{\prime}$ that

$$
\operatorname{Res}_{x_{R}}\left[\frac{\operatorname{POLRAT}\left(x_{R} ; \ldots\right)}{x_{R}^{n+1} \bar{x}_{R}^{n+R+1}}\right]=\operatorname{Res}_{x_{R}}\left[\frac{\operatorname{POLRAT}\left(x_{R} ; \ldots\right)}{x_{R}^{n+1} \bar{x}_{R}^{n+R+1}}\left(x_{R} \rightarrow \bar{x}_{R}\right)\right] .
$$

Applying $\operatorname{Res}_{x_{\pi(1)}, \ldots, x_{\pi(u-1)}}$ to both sides of the above equation establishes that $\frac{\widetilde{P}_{R}}{x_{R}^{n+1} \bar{x}_{R}^{n+R+1}}$ indeed has property (Hadas), so that $\left(\right.$ Hadas $\left._{1}\right)$ holds. This completes the proof of subsubsublemma 1.4.1.3.

Subsubsublemma 1.4.1.4: Let $\widetilde{A}_{i}$ be as in the statement of 1.4.1.2, i.e. given by $(\widetilde{A} i 1)$ and $(\widetilde{A} i 2)$ according to whether $i<R$ or $i>R$ respectively. Then:

$$
\operatorname{Res}_{x_{\pi(1)}, \ldots, x_{\pi(k)}}\left[\frac{\widetilde{A}_{i}}{1-z_{i} x_{R}}\right]=\operatorname{Res}_{x_{\pi(1)}, \ldots, x_{\pi(k)}}\left[\frac{\widetilde{A}_{i}}{1-z_{i} x_{R}}\left(x_{R} \rightarrow \bar{x}_{R}\right)\right]
$$

[ Type 'S1414 $(\mathrm{k}, \mathrm{n})$ :' in ROBBINS, for specific $\mathrm{k}$ and n.]

Proof: Recall that $u=\pi^{-1}(R)$. ( $\left.\widetilde{A} i 1\right)$ that gives $\widetilde{A}_{i}$ for $i<R$, and $(\widetilde{A} i 2)$ that gives $\widetilde{A}_{i}$ for $i>R$ are of the same form, except that the power of $z_{i}$ in the denominator of ( $\left.\widetilde{A} i 2\right)$ is 1 more (namely $n+k+2)$ than its counterpart $(n+k+1)$ in $(\widetilde{A} i 1)$. Multiplying top and bottom of $(\widetilde{A} i 1)$ by $z_{i}$ makes them exactly of the same form.

Case I: $i \in\{\pi(u+1), \ldots, \pi(k)\}$.

Let $v:=\pi^{-1}(i)$, so that $i=\pi(v)$, and $v>u$.

We have that:

$$
\frac{\widetilde{A}_{i}}{1-z_{i} x_{R}}=\frac{\operatorname{POLRAT}\left(x_{i} ; x_{\pi(1)}, \ldots, \widehat{x}_{R}, \ldots, x_{\pi(v-1)}, x_{\pi(v+1)}, \ldots, x_{\pi(k)}\right)}{x_{R}^{n+1} \bar{x}_{R}^{n+R+1} z_{i}^{n+k+2} \bar{z}_{i}^{n+i+1}\left(1-z_{i} x_{R}\right)} .
$$

Applying $R e s_{x_{\pi(v+1)}, \ldots, x_{\pi(k)}}$ to it gives:

$$
\operatorname{Res}_{x_{\pi(v+1)}, \ldots, x_{\pi(k)}}\left[\frac{\widetilde{A}_{i}}{1-z_{i} x_{R}}\right]=\frac{\operatorname{POLRAT}\left(x_{i} ; x_{\pi(1)}, \ldots, \widehat{x}_{R}, \ldots, x_{\pi(v-1)}\right)}{x_{R}^{n+1} \bar{x}_{R}^{n+R+1} z_{i}^{n+k+2} \bar{z}_{i}^{n+i+1}\left(1-z_{i} x_{R}\right)} .
$$

Applying $\operatorname{Res}_{x_{i}}$ to the above gives:

$$
\operatorname{Res}_{x_{i}, x_{\pi(v+1)}, \ldots, x_{\pi(k)}}\left[\frac{\widetilde{A}_{i}}{1-z_{i} x_{R}}\right]=\operatorname{Res}_{x_{i}}\left[\frac{\operatorname{POLRAT}\left(x_{i} ; x_{\pi(1)}, \ldots, \widehat{x}_{R}, \ldots, x_{\pi(v-1)}\right)}{x_{R}^{n+1} \bar{x}_{R}^{n+R+1} z_{i}^{n+k+2} \bar{z}_{i}^{n+i+1}\left(1-z_{i} x_{R}\right)}\right]
$$


THE Electronic Journal of COMbinatorics 3 (2) (1996), \#R13

$$
=\frac{1}{x_{R}^{n+1} \bar{x}_{R}^{n+R+1}} \cdot \operatorname{Res}_{x_{i}}\left[\frac{\operatorname{POLRAT}\left(x_{i} ; x_{\pi(1)}, \ldots, \widehat{x}_{R}, \ldots, x_{\pi(v-1)}\right)}{z_{i}^{n+k+2} \bar{z}_{i}^{n+i+1}\left(1-z_{i} x_{R}\right)}\right] .
$$

We now distinguish two subcases:

Subcase Ia: $\varepsilon_{i}=+1$, that is: $z_{i}=x_{i}$.

In this subcase, the right side of (Doron) equals:

$$
\frac{1}{x_{R}^{n+1} \bar{x}_{R}^{n+R+1}} \cdot \operatorname{Coeff} f_{x_{i}^{n+k+1}}\left[\frac{\operatorname{POLRAT}\left(x_{i} ; x_{\pi(1)}, \ldots, \widehat{x}_{R}, \ldots, x_{\pi(v-1)}\right)}{\bar{x}_{i}^{n+i+1}\left(1-x_{i} x_{R}\right)}\right] .
$$

Expanding everything involving $x_{i}$ as a power series in $x_{i}$ :

$$
\begin{gathered}
\operatorname{POLRAT}\left(x_{i} ; x_{\pi(1)}, \ldots, \widehat{x}_{R}, \ldots, x_{\pi(v-1)}\right)=\sum_{t=0}^{\text {degree }} R A T_{t}\left(x_{\pi(1)}, \ldots, \widehat{x}_{R}, \ldots, x_{\pi(v-1)}\right) x_{i}^{t}, \\
\frac{1}{\bar{x}_{i}^{n+i+1}}=\sum_{t=0}^{\infty} N U M B E R_{t} \cdot x_{i}^{t},
\end{gathered}
$$

and, most importantly:

$$
\left(1-x_{i} x_{R}\right)^{-1}=\sum_{t=0}^{\infty} x_{R}^{t} x_{i}^{t}
$$

Multiplying out, and collecting the coefficient of $x_{i}^{n+k+1}$, gives that Coef $f_{x_{i}^{n+k+1}}$ in (Gil) is a certain polynomial in $x_{R}$, of degree $\leq n+k+1$, with coefficients that are rational functions of $\left(x_{\pi(1)}, \ldots, \widehat{x}_{R}, \ldots, x_{\pi(v-1)}\right)$. In other words it is a $\operatorname{POLRAT}\left(x_{R} ; x_{\pi(1)}, \ldots, x_{\pi(v-1)}\right)$ with $\operatorname{deg}_{x_{R}} \leq$ $n+k+1$. Hence,

$\operatorname{Res}_{x_{R}, x_{\pi(u+1)}, \ldots, x_{\pi(k)}}\left[\frac{\widetilde{A}_{i}}{1-x_{i} x_{R}}\right]=\operatorname{Res}_{x_{R}, x_{\pi(u+1)}, \ldots, x_{\pi(v-1)}}\left[\frac{\operatorname{POLRAT}\left(x_{R} ; x_{\pi(1)}, \ldots, \widehat{x}_{R}, \ldots, x_{\pi(v-1)}\right)}{x_{R}^{n+1} \bar{x}_{R}^{n+R+1}}\right]$,

where the POLRAT in $\left(G i l^{\prime}\right)$ has degree $\leq n+k+1$ in $x_{R}$. Applying $\operatorname{Res}_{x_{\pi(u+1)}, \ldots, x_{\pi(v-1)}}$ to the residuand of $\left(G i l^{\prime}\right)$ gets rid of the dependence on $\left(x_{\pi(u+1)}, \ldots, x_{\pi(v-1)}\right)$, so we have:

$$
\operatorname{Res}_{x_{R}, x_{\pi(u+1)}, \ldots, x_{\pi(k)}}\left[\frac{\widetilde{A}_{i}}{1-x_{i} x_{R}}\right]=\operatorname{Res}_{x_{R}}\left[\frac{\operatorname{POLRAT}\left(x_{R} ; x_{\pi(1)}, \ldots, x_{\pi(u-1)}\right)}{x_{R}^{n+1} \bar{x}_{R}^{n+R+1}}\right], \quad\left(G i l^{\prime \prime}\right)
$$

where $\operatorname{POLRAT}\left(x_{R} ; x_{\pi(1)}, \ldots, x_{\pi(u-1)}\right)$ has degree $\leq n+k+1$ in $x_{R}$.

But, the right of $\left(G i l^{\prime \prime}\right)$ can be rewritten as:

$$
\operatorname{Res}_{x_{R}}\left[\frac{\operatorname{POLRAT}\left(x_{R} ; x_{\pi(1)}, \ldots, x_{\pi(u-1)}\right)}{x_{R}^{n+1} \bar{x}_{R}^{n+R+1}}\right]=\operatorname{Res}_{x_{R}}\left[\frac{x_{R}^{R} \cdot \operatorname{POLRAT}\left(x_{R} ; x_{\pi(1)}, \ldots, x_{\pi(u-1)}\right)}{x_{R}^{n+R+1} \bar{x}_{R}^{n+R+1}}\right] .
$$


By crucial fact $\aleph_{5}^{\prime}$, since $R+(n+k+1) \leq 2(n+R)$ (recall that always $n \geq k$ and $R \geq 1$ ), we can perform $x_{R} \rightarrow \bar{x}_{R}$ in the residuand of $\left(G i l^{\prime \prime \prime}\right)$, so

$$
\operatorname{Res}_{x_{R}, x_{\pi(u+1)}, \ldots, x_{\pi(k)}}\left[\frac{\widetilde{A}_{i}}{1-x_{i} x_{R}}\right]=\operatorname{Res}_{x_{R}, x_{\pi(u+1)}, \ldots, x_{\pi(k)}}\left[\frac{\widetilde{A}_{i}}{1-x_{i} x_{R}}\left(x_{R} \rightarrow \bar{x}_{R}\right)\right] .
$$

Applying $\operatorname{Res}_{x_{\pi(1)}, \ldots, x_{\pi(u-1)}}$ to both sides of the above equation finishes up subcase Ia of the proof of $(s u b)^{3}$ lemma 1.4.1.4.

Subcase Ib: $\varepsilon_{i}=-1$, that is: $z_{i}=\bar{x}_{i}$.

In this subcase, the right side of (Doron) equals:

$$
\frac{1}{x_{R}^{n+1} \bar{x}_{R}^{n+R+1}} \cdot \text { Coeff } x_{x_{i}^{n+i}}\left[\frac{\operatorname{POLRAT}\left(x_{i} ; x_{\pi(1)}, \ldots, \widehat{x}_{R}, \ldots, x_{\pi(v-1)}\right)}{\bar{x}_{i}^{n+k+2}\left(1-\bar{x}_{i} x_{R}\right)}\right] .
$$

Expanding everything involving $x_{i}$ as a power series in $x_{i}$ :

$$
\begin{gathered}
\operatorname{POLRAT}\left(x_{i} ; x_{\pi(1)}, \ldots, \widehat{x}_{R}, \ldots, x_{\pi(v-1)}\right)=\sum_{t=0}^{\text {degree }} R A T_{t}\left(x_{\pi(1)}, \ldots, \widehat{x}_{R}, \ldots, x_{\pi(v-1)}\right) x_{i}^{t}, \\
\frac{1}{\bar{x}_{i}^{n+k+2}}=\sum_{t=0}^{\infty} N U M B E R_{t} \cdot x_{i}^{t},
\end{gathered}
$$

and, most importantly:

$$
\left(1-\bar{x}_{i} x_{R}\right)^{-1}=\left(1-x_{R}+x_{i} x_{R}\right)^{-1}=\left(\bar{x}_{R}+x_{i} x_{R}\right)^{-1}=\sum_{t=0}^{\infty}(-1)^{t} \frac{x_{R}^{t}}{\bar{x}_{R}^{t+1}} x_{i}^{t},
$$

multiplying out, and collecting the coefficient of $x_{i}^{n+i}$, gives that $\operatorname{Coef} f_{x_{i}^{n+i}}$ in $(G i l)$ is a certain sum of terms of the form

$$
R A T_{t}\left(x_{\pi(1)}, \ldots, \widehat{x}_{R}, \ldots, x_{\pi(v-1)}\right) \frac{x_{R}^{t}}{\bar{x}_{R}^{t+1}} \quad,(t \geq 0)
$$

Hence,

$$
\operatorname{Res}_{x_{R}, x_{\pi(u+1)}, \ldots, x_{\pi(k)}}\left[\frac{\widetilde{A}_{i}}{1-\bar{x}_{i} x_{R}}\right]
$$

is a sum of terms of the forms

$$
\operatorname{Res}_{x_{R}, x_{\pi(u+1)}, \ldots, x_{\pi(v-1)}}\left[\frac{x_{R}^{t} \cdot R A T_{t}\left(x_{\pi(1)}, \ldots, \widehat{x}_{R}, \ldots, x_{\pi(v-1)}\right)}{x_{R}^{n+1} \bar{x}_{R}^{n+R+t+2}}\right],(t \geq 0) .
$$

Applying $\operatorname{Res}_{x_{\pi(u+1)}, \ldots, x_{\pi(v-1)}}$ to the residuand of $\left(G i l^{\prime}\right)$ gets rid of the dependence on $\left(x_{\pi(u+1)}, \ldots, x_{\pi(v-1)}\right)$, so we have that

$$
\operatorname{Res}_{x_{R}, x_{\pi(u+1)}, \ldots, x_{\pi(k)}}\left[\frac{\widetilde{A}_{i}}{1-\bar{x}_{i} x_{R}}\right]
$$


is a sum of terms of the form

$$
\operatorname{Res}_{x_{R}}\left[\frac{x_{R}^{t} R A T_{t}\left(x_{\pi(1)}, \ldots, x_{\pi(u-1)}\right)}{x_{R}^{n+1} \bar{x}_{R}^{n+R+t+2}}\right],(t \geq 0)
$$

But, each such term can be written as

$$
R A T_{t}\left(x_{\pi(1)}, \ldots, x_{\pi(u-1)}\right) \operatorname{Res}_{x_{R}}\left[\frac{x_{R}^{R+2 t+1}}{x_{R}^{n+R+t+2} \bar{x}_{R}^{n+R+t+2}}\right] \quad, \quad(t \geq 0)
$$

By crucial fact $\aleph_{5}^{\prime}$, since $R+2 t+1 \leq 2(n+R+t+1)$, we can perform $x_{R} \rightarrow \bar{x}_{R}$ in the residuand of $\left(G i l^{\prime \prime \prime}\right)$, so

$$
\operatorname{Res}_{x_{R}, x_{\pi(u+1)}, \ldots, x_{\pi(k)}}\left[\frac{\widetilde{A}_{i}}{1-\bar{x}_{i} x_{R}}\right]=\operatorname{Res}_{x_{R}, x_{\pi(u+1)}, \ldots, x_{\pi(k)}}\left[\frac{\widetilde{A}_{i}}{1-\bar{x}_{i} x_{R}}\left(x_{R} \rightarrow \bar{x}_{R}\right)\right] .
$$

Applying $\operatorname{Res}_{x_{\pi(1)}, \ldots, x_{\pi(u-1)}}$ to both sides of the above equation finishes up subcase Ib of the proof of subsubsublemma 1.4.1.4.

Case II: $i \in\{\pi(1), \ldots, \pi(u-1)\}$.

Let $v:=\pi^{-1}(i)$, so that $i=\pi(v)$, and $v<u$. By the definition of $u, \varepsilon_{i}$ must be equal to +1 , so that $z_{i}=x_{i}$.

The left side of $\left(\mathrm{Hadas}_{2}\right)$ (in the statement of $(s u b)^{3}$ lemma 1.4.1.4, which we are currently proving,) can be written as:

$$
\operatorname{Res}_{x_{\pi(1)}, \ldots, x_{\pi(k)}}\left[\frac{\widetilde{A}_{i}}{1-x_{i} x_{R}}\right]=\operatorname{Res}_{x_{\pi(1)}, \ldots, x_{\pi(v-1)}, x_{i}, x_{\pi(v+1)}, \ldots, x_{\pi(u-1)}, x_{R}, x_{\pi(u+1)}, \ldots, x_{\pi(k)}}\left[\frac{\widetilde{A}_{i}}{1-x_{i} x_{R}}\right] .
$$

The next $(s u b)^{4}$ lemma assures us that, on the right side of (Ruthie), in the order of residue taking $R e s_{x_{\pi(1)}, \ldots, x_{\pi(k)}}$, it is permissible to transpose $x_{i}$ and $x_{R}$, without affecting its value, and likewise when the residuand is replaced by $\frac{\widetilde{A}_{i}}{1-x_{i} x_{R}}\left(x_{R} \rightarrow \bar{x}_{R}\right)$.

Subsubsubsublemma 1.4.1.4.1:

$$
\begin{array}{r}
\operatorname{Res}_{x_{\pi(1)}, \ldots, x_{\pi(v-1)}, x_{i}, x_{\pi(v+1)}, \ldots, x_{\pi(u-1)}, x_{R}, x_{\pi(u+1)}, \ldots, x_{\pi(k)}}\left[\frac{\widetilde{A}_{i}}{1-x_{i} x_{R}}\right] \\
=\operatorname{Res}_{x_{\pi(1)}, \ldots, x_{\pi(v-1)}, x_{R}, x_{\pi(v+1)}, \ldots, x_{\pi(u-1)}, x_{i}, x_{\pi(u+1)}, \ldots, x_{\pi(k)}}\left[\frac{\widetilde{A}_{i}}{1-x_{i} x_{R}}\right],
\end{array}
$$

and

$$
\operatorname{Res}_{x_{\pi(1)}, \ldots, x_{\pi(v-1)}, x_{i}, x_{\pi(v+1)}, \ldots, x_{\pi(u-1)}, x_{R}, x_{\pi(u+1)}, \ldots, x_{\pi(k)}}\left[\frac{\widetilde{A}_{i}}{1-x_{i} x_{R}}\left(x_{R} \rightarrow \bar{x}_{R}\right)\right]
$$


THE Electronic Journal of COMBinatorics 3 (2) (1996), \#R13

$$
=\operatorname{Res}_{x_{\pi(1)}, \ldots, x_{\pi(v-1)}, x_{R}, x_{\pi(v+1)}, \ldots, x_{\pi(u-1)}, x_{i}, x_{\pi(u+1)}, \ldots, x_{\pi(k)}}\left[\frac{\widetilde{A}_{i}}{1-x_{i} x_{R}}\left(x_{R} \rightarrow \bar{x}_{R}\right)\right] .
$$

We will only prove $($ Noam $)$, since the proof of $(\overline{\text { Noam }})$ goes verbatim. Changing the order of residue-taking from $\operatorname{Res}_{x_{\pi(1)}, \ldots, x_{i}, \ldots, x_{R}, \ldots, x_{\pi(k)}}$ to $\operatorname{Res}_{x_{\pi(1)}, \ldots, x_{R}, \ldots, x_{i}, \ldots, x_{\pi(k)}}$ is permitted by crucial fact $\aleph_{4}$, provided the residuand of $\operatorname{Res}_{x_{\pi(1)}, \ldots, x_{i}, \ldots, x_{R}}$ on the left side of (Noam), namely,

$$
\operatorname{Res}_{x_{\pi(u+1)}, \ldots, x_{\pi(k)}}\left[\frac{\widetilde{A}_{i}}{1-x_{i} x_{R}}\right] \text {, }
$$

possesses a bona fide Laurent expansion in its variables $\left(x_{\pi(1)}, \ldots, x_{i}, \ldots, x_{\pi(u-1)}, x_{R}\right)$, i.e. that it is a rational function whose denominator is a monomial times a polynomial with a non-zero constant term.

This fact would follow, by invoking the case $w=u$, from the following $(s u b)^{5}$ lemma.

Subsubsubsubsublemma 1.4.1.4.1.1 : Let $u \leq w \leq k$, and $\pi^{-1}(i)<u$, then

$$
\operatorname{Res}_{x_{\pi(w+1)}, \ldots, x_{\pi(k)}}\left[\frac{\widetilde{A}_{i}}{1-x_{i} x_{R}}\right]
$$

can be expressed as a sum of terms of the form

$$
\frac{\operatorname{POL}\left(x_{\pi(1)}, \ldots, \widehat{x}_{R}, \ldots, x_{\pi(w)}\right)}{1-x_{i} x_{R}} \cdot \prod_{j=1}^{w} \frac{1}{z_{\pi(j)}^{\alpha_{j}} \bar{z}_{\pi(j)}^{\beta_{j}}} \cdot \prod_{\substack{r, s \in\{\pi(1), \ldots, \pi(w)\} \\ r<s ; r, s \neq R, i}} \frac{1}{\left(1-z_{r} z_{s}\right)\left(1-\bar{z}_{r} z_{s}\right)}
$$

Proof: The proof is by decreasing induction on $w$. When $w=k$ then this follows tautologically from $(\widetilde{A} i 1)$ and $(\widetilde{A} i 2)$. Suppose the statement of the $(s u b)^{5}$ lemma is true when $w$ is replaced by $w+1$, and let's deduce from it that it is true for $w$ proper.

By the inductive hypothesis, the quantity of interest is a sum of terms of the form

$$
\operatorname{Res}_{x_{\pi(w+1)}}\left[\frac{\operatorname{POL}\left(x_{\pi(1)}, \ldots, \widehat{x}_{R}, \ldots, x_{\pi(w)}, x_{\pi(w+1)}\right)}{1-x_{i} x_{R}} \cdot \prod_{j=1}^{w+1} \frac{1}{z_{\pi(j)}^{\alpha_{j}} \bar{z}_{\pi(j)}^{\beta_{j}}} \cdot \prod_{\substack{r, s \in\{\pi(1), \ldots, \pi(w+1)\} \\ r<s ; r, s \neq R, i}} \frac{1}{\left(1-z_{r} z_{s}\right)\left(1-\bar{z}_{r} z_{s}\right)}\right] .
$$

Let's abbreviate $\pi(w+1)$ to $S, \alpha_{w+1}$ to $\alpha$ and $\beta_{w+1}$ to $\beta$. Then the above can be rewritten as:

$$
\begin{gathered}
\frac{1}{1-x_{i} x_{R}} \prod_{j=1}^{w} \frac{1}{z_{\pi(j)}^{\alpha_{j}} \bar{z}_{\pi(j)}^{\beta_{j}}} \cdot \prod_{\substack{r, s \in\{\pi(1), \ldots, \pi(w)\} \\
r<s ; r, s \neq R, i}} \frac{1}{\left(1-z_{r} z_{s}\right)\left(1-\bar{z}_{r} z_{s}\right)} \cdot \operatorname{Res}_{x_{S}}[ \\
\left.\frac{\operatorname{POL}\left(x_{\pi(1)}, \ldots, \widehat{x}_{R}, \ldots, x_{\pi(w)}, x_{S}\right)}{z_{S}^{\alpha} \bar{z}_{S}^{\beta}} \prod_{\substack{r \in\{\pi(1), \ldots, \pi(w)\} \\
r \neq S, R, i}} \frac{1}{1-z_{r} z_{S}} \prod_{\substack{r \in\{\pi(1), \ldots, \pi(w)\} \\
r<S ; r \neq R, i}} \frac{1}{1-\bar{z}_{r} z_{S}} \prod_{\substack{r \in\{\pi(1), \ldots, \pi(w)\} \\
r>S ; r \neq R, i}} \frac{1}{1-\bar{z}_{S} z_{r}}\right] .
\end{gathered}
$$


$R e s_{x_{S}}[\ldots]$ above can be rewritten as:

$$
\begin{gathered}
\operatorname{Coeff}_{\left.x_{S}^{(\alpha} \text { or } \beta\right)-1} \\
{\left[\frac{\operatorname{POL}\left(x_{\pi(1)}, \ldots, \widehat{x}_{R}, \ldots, x_{\pi(w)}, x_{S}\right)}{\bar{x}_{S}^{(\beta \text { or } \alpha)}} \prod_{\substack{r \in\{\pi(1), \ldots, \pi(w)\} \\
r \neq S, R, i}} \frac{1}{1-z_{r} z_{S}} \prod_{\substack{r \in\{\pi(1), \ldots, \pi(w)\} \\
r<S ; r \neq R, i}} \frac{1}{1-\bar{z}_{r} z_{S}} \prod_{\substack{r \in\{\pi(1), \ldots, \pi(w)\} \\
r>S ; r \neq R, i \\
\text { ration }}} \frac{1}{1-\bar{z}_{S} z_{r}}\right] .}
\end{gathered}
$$

(Danny)

Expanding everything in powers of $x_{S}$, using

$$
\begin{gathered}
\operatorname{POL}\left(x_{\pi(1)}, \ldots, \widehat{x}_{R}, \ldots, x_{\pi(w)}, x_{S}\right)=\sum_{t=0}^{\text {finite }} P O L_{t}\left(x_{\pi(1)}, \ldots, \widehat{x}_{R}, \ldots, x_{\pi(w)}\right) x_{S}^{t}, \\
\frac{1}{1-x_{S} z_{r}}=\sum_{t=0}^{\infty} z_{r}^{t} x_{S}^{t} \quad, \quad \frac{1}{1-x_{S} \bar{z}_{r}}=\sum_{t=0}^{\infty} \bar{z}_{r}^{t} x_{S}^{t},
\end{gathered}
$$

and/or

$$
\frac{1}{1-\bar{x}_{S} z_{r}}=\frac{1}{\bar{z}_{r}+z_{r} x_{S}}=\sum_{t=0}^{\infty} \frac{(-1)^{t} z_{r}^{t}}{\bar{z}_{r}^{t+1}} x_{S}^{t} \quad, \quad \frac{1}{1-\bar{x}_{S} \bar{z}_{r}}=\frac{1}{z_{r}+\bar{z}_{r} x_{S}}=\sum_{t=0}^{\infty} \frac{(-1)^{t} \bar{z}_{r}^{t}}{z_{r}^{t+1}} x_{S}^{t}
$$

for $r \in\{\pi(1), \ldots, \pi(w)\} \backslash\{i, R\}$, as well as,

$$
\frac{1}{\bar{x}_{S}^{\text {number }}}=\sum_{t=0}^{\infty} N U M B E R_{t} \cdot x_{S}^{t}
$$

multiplying out, and then taking the appropriate coefficient of $x_{S}$ in (Danny), shows that indeed the statement of the $(s u b)^{5}$ lemma also holds with $w$, except that the previous sum (in the case $w+1$ ) has been replaced by a much larger, but still finite, sum. This completes the proof of $(s u b)^{5}$ lemma 1.4.1.4.1.1.

Going back to the proof of $(s u b)^{4}$ lemma 1.4.1.4.1, we employ the above $(s u b)^{5}$ lemma with its lowest case, $w=u$. By the definition of $u, \varepsilon_{\pi(1)}=\ldots, \varepsilon_{\pi(u-1)}=+1$, so that $z_{\pi(1)}=x_{\pi(1)}, \ldots, z_{\pi(u-1)}=$ $x_{\pi(u-1)}$, and we see that

$$
\operatorname{Res}_{x_{\pi(u+1)}, \ldots, x_{\pi(k)}}\left[\frac{\widetilde{A}_{i}}{1-x_{i} x_{R}}\right]
$$

can be expressed as a sum of terms of the form

$$
\frac{\operatorname{POL}\left(x_{\pi(1)}, \ldots, x_{\pi(u-1)}\right)}{1-x_{R} x_{i}} \cdot \prod_{j=1}^{u} \frac{1}{x_{\pi(j)}^{\alpha_{j}} \bar{x}_{\pi(j)}^{\beta_{j}}} \cdot \prod_{\substack{r, s \in\{\pi(1), \ldots, \pi(u-1)\} \\ r<s ; r, s \neq i}} \frac{1}{\left(1-x_{r} x_{s}\right)\left(1-\bar{x}_{r} x_{s}\right)}
$$

each of which possesses a genuine Laurent series in its arguments $\left(x_{\pi(1)}, \ldots, x_{\pi(u)}\right)$, so for each of these, by $\aleph_{4}^{\prime}$, applying Res $\ldots, x_{i}, \ldots, x_{R}$ to them is the same as applying $R e s_{\ldots, x_{R}, \ldots, x_{i}}$, and this finishes up the proof of $(s u b)^{4}$ lemma 1.4.1.4.1. 
Going back to the proof of Case II of $(s u b)^{3}$ lemma 1.4.1.4, we use $(s u b)^{4}$ lemma 1.4.1.4.1 to change the right side of (Ruthie) to:

$$
\operatorname{Res}_{x_{\pi(1)}, \ldots, x_{\pi(v-1)}, x_{R}, x_{\pi(v+1)}, \ldots, x_{\pi(u-1)}, x_{i}, x_{\pi(u+1)}, \ldots, x_{\pi(k)}}\left[\frac{\widetilde{A}_{i}}{1-x_{i} x_{R}}\right] .
$$

$($ Ruthie $)$

But this is identical to the form treated by subcase Ia, which says that this is the same as:

$$
\left.\operatorname{Res}_{x_{\pi(1)}, \ldots, x_{\pi(v-1)}, x_{R}, x_{\pi(v+1)}, \ldots, x_{\pi(u-1)}, x_{i}, x_{\pi(u+1)}, \ldots, x_{\pi(k)}}\left[\frac{\widetilde{A}_{i}}{1-x_{i} x_{R}}\left(x_{R} \rightarrow \bar{x}_{R}\right)\right] . \quad \text { (Ruthie } e^{\prime \prime}\right)
$$

Now, we use the second part of $(s u b)^{4}$ lemma 1.4.1.4.1, i.e. $(\overline{N o a m})$ (whose proof was omitted since it was identical to that of $($ Noam $)$ ), to change this into

$$
\operatorname{Res}_{x_{\pi(1)}, \ldots, x_{\pi(v-1)}, x_{i}, x_{\pi(v+1)}, \ldots, x_{\pi(u-1)}, x_{R}, x_{\pi(u+1)}, \ldots, x_{\pi(k)}}\left[\frac{\widetilde{A}_{i}}{1-x_{i} x_{R}}\left(x_{R} \rightarrow \bar{x}_{R}\right)\right],
$$

which was exactly what we had to show in order to complete the proof of Case II of $(s u b)^{3}$ lemma 1.4.1.4. This completes the proof of $(s u b)^{3}$ lemma 1.4.1.4.

Subsubsublemma 1.4.1.5: Let $\widetilde{B}_{i},(1 \leq i<R)$, be as in the statement of 1.4 .1 .2 , i.e. given by $(\widetilde{B} i 1)$. Then

$$
\operatorname{Res}_{x_{\pi(1)}, \ldots, x_{\pi(k)}}\left[\frac{\widetilde{B}_{i}}{1-\bar{z}_{i} x_{R}}\right]=\operatorname{Res}_{x_{\pi(1)}, \ldots, x_{\pi(k)}}\left[\frac{\widetilde{B}_{i}}{1-\bar{z}_{i} x_{R}}\left(x_{R} \rightarrow \bar{x}_{R}\right)\right] .
$$

[ Type 'S1415 $(\mathrm{k}, \mathrm{n})$ :' in ROBBINS, for specific $\mathrm{k}$ and $\mathrm{n}$.

\section{Proof:}

Case I: $i \in\{\pi(u+1), \ldots, \pi(k)\}$.

Let $v:=\pi^{-1}(i)$, so that $i=\pi(v)$, and $v>u$. We have that:

$$
\frac{\widetilde{B}_{i}}{1-\bar{z}_{i} x_{R}}=\frac{\operatorname{POLRAT}\left(x_{i} ; x_{\pi(1)}, \ldots, \widehat{x}_{R}, \ldots, x_{\pi(v-1)}, x_{\pi(v+1)}, \ldots, x_{\pi(k)}\right)}{x_{R}^{n+1} \bar{x}_{R}^{n+R+1} z_{i}^{n+1} \bar{z}_{i}^{n+i+k+1}\left(1-\bar{z}_{i} x_{R}\right)} .
$$

Applying $R e s_{x_{\pi(v+1)}, \ldots, x_{\pi(k)}}$ to it gives:

$$
\operatorname{Res}_{x_{\pi(v+1)}, \ldots, x_{\pi(k)}}\left[\frac{\widetilde{B}_{i}}{1-\bar{z}_{i} x_{R}}\right]=\frac{\operatorname{POLRAT}\left(x_{i} ; x_{\pi(1)}, \ldots, \widehat{x}_{R}, \ldots, x_{\pi(v-1)}\right)}{x_{R}^{n+1} \bar{x}_{R}^{n+R+1} z_{i}^{n+1} \bar{z}_{i}^{n+i+k+1}\left(1-\bar{z}_{i} x_{R}\right)} .
$$

Applying $\operatorname{Res}_{x_{i}}$ to the above gives:

$$
\operatorname{Res}_{x_{i}, x_{\pi(v+1)}, \ldots, x_{\pi(k)}}\left[\frac{\widetilde{B}_{i}}{1-\bar{z}_{i} x_{R}}\right]=\operatorname{Res}_{x_{i}}\left[\frac{\operatorname{POLRAT}\left(x_{i} ; x_{\pi(1)}, \ldots, \widehat{x}_{R}, \ldots, x_{\pi(v-1)}\right)}{x_{R}^{n+1} \bar{x}_{R}^{n+R+1} z_{i}^{n+1} \bar{z}_{i}^{n+i+k+1}\left(1-\bar{z}_{i} x_{R}\right)}\right]
$$


THE Electronic Journal of COMbinatorics 3 (2) (1996), \#R13

$$
=\frac{1}{x_{R}^{n+1} \bar{x}_{R}^{n+R+1}} \cdot \operatorname{Res}_{x_{i}}\left[\frac{\operatorname{POLRAT}\left(x_{i} ; x_{\pi(1)}, \ldots, \widehat{x}_{R}, \ldots, x_{\pi(v-1)}\right)}{z_{i}^{n+1} \bar{z}_{i}^{n+i+k+1}\left(1-\bar{z}_{i} x_{R}\right)}\right] .
$$

We now distinguish two subcases:

Subcase Ia: $\varepsilon_{i}=-1$, that is: $\bar{z}_{i}=x_{i}$ (i.e. $\left.z_{i}=\bar{x}_{i}\right)$.

In this subcase, the right side of (Doron) equals:

$$
\frac{1}{x_{R}^{n+1} \bar{x}_{R}^{n+R+1}} \cdot \operatorname{Coeff} f_{x_{i}^{n+i+k}}\left[\frac{\operatorname{POLRAT}\left(x_{i} ; x_{\pi(1)}, \ldots, \widehat{x}_{R}, \ldots, x_{\pi(v-1)}\right)}{\bar{x}_{i}^{n+1}\left(1-x_{i} x_{R}\right)}\right]
$$

Expanding everything involving $x_{i}$ as a power series in $x_{i}$ :

$$
\begin{gathered}
\operatorname{POLRAT}\left(x_{i} ; x_{\pi(1)}, \ldots, \widehat{x}_{R}, \ldots, x_{\pi(v-1)}\right)=\sum_{t=0}^{\text {degree }} R A T_{t}\left(x_{\pi(1)}, \ldots, \widehat{x}_{R}, \ldots, x_{\pi(v-1)}\right) x_{i}^{t}, \\
\frac{1}{\bar{x}_{i}^{n+1}}=\sum_{t=0}^{\infty} N U M B E R_{t} \cdot x_{i}^{t},
\end{gathered}
$$

and, most importantly:

$$
\left(1-x_{i} x_{R}\right)^{-1}=\sum_{t=0}^{\infty} x_{R}^{t} x_{i}^{t}
$$

multiplying out, and collecting the coefficient of $x_{i}^{n+i+k}$, gives that Coef $f_{x_{i}^{n+i+k}}$ in (Gil) is a certain polynomial in $x_{R}$, of degree $\leq n+i+k$, with coefficients that are rational functions of $\left(x_{\pi(1)}, \ldots, \widehat{x}_{R}, \ldots, x_{\pi(v-1)}\right)$. In other words it is a $\operatorname{POLRAT}\left(x_{R} ; x_{\pi(1)}, \ldots, x_{\pi(v-1)}\right)$ with $\operatorname{deg}_{x_{R}} \leq$ $n+i+k$. Hence,

$\operatorname{Res}_{x_{R}, x_{\pi(u+1)}, \ldots, x_{\pi(k)}}\left[\frac{\widetilde{B}_{i}}{1-x_{i} x_{R}}\right]=\operatorname{Res}_{x_{R}, x_{\pi(u+1)}, \ldots, x_{\pi(v-1)}}\left[\frac{\operatorname{POLRAT}\left(x_{R} ; x_{\pi(1)}, \ldots, \widehat{x}_{R}, \ldots, x_{\pi(v-1)}\right)}{x_{R}^{n+1} \bar{x}_{R}^{n+R+1}}\right]$,

where the POLRAT in $\left(G i l^{\prime}\right)$ has degree $\leq n+i+k$ in $x_{R}$. Applying $\operatorname{Res}_{x_{\pi(u+1)}, \ldots, x_{\pi(v-1)}}$ to the residuand of $\left(G i l^{\prime}\right)$ gets rid of the dependence on $\left(x_{\pi(u+1)}, \ldots, x_{\pi(v-1)}\right)$, so we have:

$$
\operatorname{Res}_{x_{R}, x_{\pi(u+1)}, \ldots, x_{\pi(k)}}\left[\frac{\widetilde{B}_{i}}{1-x_{i} x_{R}}\right]=\operatorname{Res}_{x_{R}}\left[\frac{\operatorname{POLRAT}\left(x_{R} ; x_{\pi(1)}, \ldots, x_{\pi(u-1)}\right)}{x_{R}^{n+1} \bar{x}_{R}^{n+R+1}}\right]
$$

where $\operatorname{POLRAT}\left(x_{R} ; x_{\pi(1)}, \ldots, x_{\pi(u-1)}\right)$ has degree $\leq n+i+k$ in $x_{R}$.

But, the right of $\left(G i l^{\prime \prime}\right)$ can be rewritten as:

$$
\operatorname{Res}_{x_{R}}\left[\frac{x_{R}^{R} \cdot \operatorname{POLRAT}\left(x_{R} ; x_{\pi(1)}, \ldots, x_{\pi(u-1)}\right)}{x_{R}^{n+R+1} \bar{x}_{R}^{n+R+1}}\right] .
$$


By crucial fact $\aleph_{5}^{\prime}$, since $R+(n+i+k) \leq 2(n+R)$ (recall that always $n \geq k$ and $i<R$ ), we can perform $x_{R} \rightarrow \bar{x}_{R}$ in the residuand of $\left(G i l^{\prime \prime \prime}\right)$, so

$$
\operatorname{Res}_{x_{R}, x_{\pi(u+1)}, \ldots, x_{\pi(k)}}\left[\frac{\widetilde{B}_{i}}{1-x_{i} x_{R}}\right]=\operatorname{Res}_{x_{R}, x_{\pi(u+1)}, \ldots, x_{\pi(k)}}\left[\frac{\widetilde{B}_{i}}{1-x_{i} x_{R}}\left(x_{R} \rightarrow \bar{x}_{R}\right)\right] .
$$

Applying $\operatorname{Res}_{x_{\pi(1)}, \ldots, x_{\pi(u-1)}}$ to both sides of the above equation finishes up subcase Ia of the proof of subsublemma 1.4.1.5.

Subcase Ib: $\varepsilon_{i}=1$, that is: $\bar{z}_{i}=\bar{x}_{i}\left(\right.$ i.e. $\left.z_{i}=x_{i}\right)$.

In this subcase, the right side of (Doron) equals:

$$
\frac{1}{x_{R}^{n+1} \bar{x}_{R}^{n+R+1}} \cdot \text { Coeff }_{x_{i}^{n}}\left[\frac{\operatorname{POLRAT}\left(x_{i} ; x_{\pi(1)}, \ldots, \widehat{x}_{R}, \ldots, x_{\pi(v-1)}\right)}{\bar{x}_{i}^{n+i+k+1}\left(1-\bar{x}_{i} x_{R}\right)}\right] .
$$

Expanding everything involving $x_{i}$ as a power series in $x_{i}$ :

$$
\begin{gathered}
\operatorname{POLRAT}\left(x_{i} ; x_{\pi(1)}, \ldots, \widehat{x}_{R}, \ldots, x_{\pi(v-1)}\right)=\sum_{t=0}^{\text {degree }} R A T_{t}\left(x_{\pi(1)}, \ldots, \widehat{x}_{R}, \ldots, x_{\pi(v-1)}\right) x_{i}^{t}, \\
\frac{1}{\bar{x}_{i}^{n+i+k+1}}=\sum_{t=0}^{\infty} N U M B E R_{t} \cdot x_{i}^{t},
\end{gathered}
$$

and, most importantly:

$$
\left(1-\bar{x}_{i} x_{R}\right)^{-1}=\left(1-x_{R}+x_{i} x_{R}\right)^{-1}=\left(\bar{x}_{R}+x_{i} x_{R}\right)^{-1}=\sum_{t=0}^{\infty}(-1)^{t} \frac{x_{R}^{t}}{\bar{x}_{R}^{t+1}} x_{i}^{t},
$$

multiplying out, and collecting the coefficient of $x_{i}^{n}$, gives that Coef $f_{x_{i}^{n}}$ in $(G i l)$ is a certain sum of terms of the form

$$
R A T_{t}\left(x_{\pi(1)}, \ldots, \widehat{x}_{R}, \ldots, x_{\pi(v-1)}\right) \frac{x_{R}^{t}}{\bar{x}_{R}^{t+1}} \quad,(t \geq 0)
$$

Hence,

$$
\operatorname{Res}_{x_{R}, x_{\pi(u+1)}, \ldots, x_{\pi(k)}}\left[\frac{\widetilde{B}_{i}}{1-\bar{x}_{i} x_{R}}\right]
$$

is a sum of terms of the forms

$$
\operatorname{Res}_{x_{R}, x_{\pi(u+1)}, \ldots, x_{\pi(v-1)}}\left[\frac{x_{R}^{t} \cdot R A T_{t}\left(x_{\pi(1)}, \ldots, \widehat{x}_{R}, \ldots, x_{\pi(v-1)}\right)}{x_{R}^{n+1} \bar{x}_{R}^{n+R+t+2}}\right],(t \geq 0) .
$$

Applying $\operatorname{Res}_{x_{\pi(u+1)}, \ldots, x_{\pi(v-1)}}$ to the residuand of $\left(G i l^{\prime}\right)$ gets rid of the dependence on $\left(x_{\pi(u+1)}, \ldots, x_{\pi(v-1)}\right)$, so we have that

$$
\operatorname{Res}_{x_{R}, x_{\pi(u+1)}, \ldots, x_{\pi(k)}}\left[\frac{\widetilde{B}_{i}}{1-\bar{x}_{i} x_{R}}\right]
$$


is a sum of terms of the form

$$
\operatorname{Res}_{x_{R}}\left[\frac{x_{R}^{t} R A T_{t}\left(x_{\pi(1)}, \ldots, x_{\pi(u-1)}\right)}{x_{R}^{n+1} \bar{x}_{R}^{n+R+t+2}}\right],(t \geq 0)
$$

But, each such term can be written as

$$
R A T_{t}\left(x_{\pi(1)}, \ldots, x_{\pi(u-1)}\right) \cdot \operatorname{Res}_{x_{R}}\left[\frac{x_{R}^{R+2 t+1}}{x_{R}^{n+R+t+2} \bar{x}_{R}^{n+R+t+2}}\right] \quad, \quad(t \geq 0)
$$

By crucial fact $\aleph_{5}^{\prime}$, since $R+2 t+1 \leq 2(n+R+t+1)$, we can perform $x_{R} \rightarrow \bar{x}_{R}$ in the residuand of $\left(G i l^{\prime \prime \prime}\right)$, so

$$
\operatorname{Res}_{x_{R}, x_{\pi(u+1)}, \ldots, x_{\pi(k)}}\left[\frac{\widetilde{B}_{i}}{1-\bar{x}_{i} x_{R}}\right]=\operatorname{Res}_{x_{R}, x_{\pi(u+1)}, \ldots, x_{\pi(k)}}\left[\frac{\widetilde{B}_{i}}{1-\bar{x}_{i} x_{R}}\left(x_{R} \rightarrow \bar{x}_{R}\right)\right] .
$$

Applying $\operatorname{Res}_{x_{\pi(1)}, \ldots, x_{\pi(u-1)}}$ to both sides of the above equation finishes up subcase Ib of the proof of subsubsublemma 1.4.1.5.

Case II: $i \in\{\pi(1), \ldots, \pi(u-1)\}$.

Let $v:=\pi^{-1}(i)$, so that $i=\pi(v)$, and $v<u$. By the definition of $u, \varepsilon_{i}$ must be equal to +1 , so that $z_{i}=x_{i}$.

The left side of $\left(\mathrm{Hadas}_{3}\right)$ can be written as:

$$
\operatorname{Res}_{x_{\pi(1)}, \ldots, x_{\pi(k)}}\left[\frac{\widetilde{B}_{i}}{1-\bar{x}_{i} x_{R}}\right]=\operatorname{Res}_{x_{\pi(1)}, \ldots, x_{\pi(v-1)}, x_{i}, x_{\pi(v+1)}, \ldots, x_{\pi(u-1)}, x_{R}, x_{\pi(u+1)}, \ldots, x_{\pi(k)}}\left[\frac{\widetilde{B}_{i}}{1-\bar{x}_{i} x_{R}}\right]_{\text {(Ruthie) }} .
$$

The first step is to 'unbar' the $x_{i}$ in (Ruthie).

Subsubsubsublemma 1.4.1.5.1: Let $\widetilde{B}_{i},(1 \leq i<R)$, be as in the statement of 1.4.1.2, i.e. given by $(\widetilde{B} i 1)$. (Recall that $\pi$ is an arbitrary permutation, $\pi(u)=R, \pi(v)=i$ and $v<u$.) Then

$$
\begin{gathered}
\operatorname{Res}_{x_{\pi(1)}, \ldots, x_{\pi(k)}}\left[\frac{\widetilde{B}_{i}\left(x_{i} \rightarrow \bar{x}_{i}\right)}{1-x_{i} x_{R}}\right]=\operatorname{Res}_{x_{\pi(1)}, \ldots, x_{\pi(k)}}\left[\frac{\widetilde{B}_{i}}{1-\bar{x}_{i} x_{R}}\right] . \\
\operatorname{Res}_{x_{\pi(1)}, \ldots, x_{\pi(k)}}\left[\frac{\widetilde{B}_{i}\left(x_{i} \rightarrow \bar{x}_{i}, x_{R} \rightarrow \bar{x}_{R}\right)}{1-x_{i} \bar{x}_{R}}\right]=\operatorname{Res}_{x_{\pi(1)}, \ldots, x_{\pi(k)}}\left[\frac{\widetilde{B}_{i}\left(x_{R} \rightarrow \bar{x}_{R}\right)}{1-\bar{x}_{i} \bar{x}_{R}}\right] .
\end{gathered}
$$

Proof: The proof of (Michal) and $\left(\mathrm{Michal}^{\prime}\right)$ are very similar to subcases Ia and Ib respectively, except that the roles of $i$ and $R$ are reversed. We will only prove (Michal), since $\left(M i c h a l^{\prime}\right)$ is almost identical to the proof of subcase Ib (with $i$ and $R$ interchanged.) 
We have that:

$$
\frac{\widetilde{B}_{i}\left(x_{i} \rightarrow \bar{x}_{i}\right)}{1-x_{i} x_{R}}=\frac{\operatorname{POLRAT}\left(x_{i} ; x_{\pi(1)}, \ldots, \widehat{x}_{R}, \ldots, x_{\pi(v-1)}, x_{\pi(v+1)}, \ldots, x_{\pi(k)}\right)}{x_{R}^{n+1} \bar{x}_{R}^{n+R+1} \bar{x}_{i}^{n+1} x_{i}^{n+i+k+1}\left(1-x_{i} x_{R}\right)}
$$

Applying $\operatorname{Res}_{x_{\pi(u+1)}, \ldots, x_{\pi(k)}}$ to it gives:

$$
\operatorname{Res}_{x_{\pi(u+1)}, \ldots, x_{\pi(k)}}\left[\frac{\widetilde{B}_{i}\left(x_{i} \rightarrow \bar{x}_{i}\right)}{1-x_{i} x_{R}}\right]=\frac{\operatorname{POLRAT}\left(x_{i} ; x_{\pi(1)}, \ldots, \widehat{x}_{i}, \ldots, x_{\pi(u-1)}\right)}{x_{R}^{n+1} \bar{x}_{R}^{n+R+1} \bar{x}_{i}^{n+1} x_{i}^{n+i+k+1}\left(1-x_{i} x_{R}\right)} .
$$

Applying $R e s_{x_{R}}$ to the above gives:

$$
\begin{aligned}
& \operatorname{Res}_{x_{R}, x_{\pi(u+1)}, \ldots, x_{\pi(k)}}\left[\frac{\widetilde{B}_{i}\left(x_{i} \rightarrow \bar{x}_{i}\right)}{1-x_{i} x_{R}}\right]=\operatorname{Res}_{x_{R}}\left[\frac{\operatorname{POLRAT}\left(x_{i} ; x_{\pi(1)}, \ldots, \widehat{x}_{i}, \ldots, x_{\pi(u-1)}\right)}{x_{R}^{n+1} \bar{x}_{R}^{n+R+1} \bar{x}_{i}^{n+1} x_{i}^{n+i+k+1}\left(1-x_{i} x_{R}\right)}\right] \\
& =\frac{\operatorname{POLRAT}\left(x_{i} ; x_{\pi(1)}, \ldots, \widehat{x}_{i}, \ldots, x_{\pi(u-1)}\right)}{x_{i}^{n+i+k+1} \bar{x}_{i}^{n+1}} \cdot \operatorname{Res}_{x_{R}}\left[\frac{1}{x_{R}^{n+1} \bar{x}_{R}^{n+R+1}\left(1-x_{i} x_{R}\right)}\right] \cdot \quad(H
\end{aligned}
$$

The right side of (Herb) equals:

$$
\frac{\operatorname{POLRAT}\left(x_{i} ; x_{\pi(1)}, \ldots, \widehat{x}_{i}, \ldots, x_{\pi(u-1)}\right)}{x_{i}^{n+i+k+1} \bar{x}_{i}^{n+1}} \cdot \operatorname{Coeff} f_{x_{R}^{n}}\left[\frac{1}{\bar{x}_{R}^{n+R+1}\left(1-x_{i} x_{R}\right)}\right] .
$$

Expanding the two quantities involving $x_{R}$ above as a power series in $x_{R}$ :

$$
\frac{1}{\bar{x}_{R}^{n+R+1}}=\sum_{t=0}^{\infty} N U M B E R_{t} \cdot x_{R}^{t}
$$

and, more importantly:

$$
\left(1-x_{i} x_{R}\right)^{-1}=\sum_{t=0}^{\infty} x_{i}^{t} x_{R}^{t}
$$

multiplying out, and collecting the coefficient of $x_{R}^{n}$, gives that Coef $f_{x_{R}^{n}}$ in (David) is a certain polynomial in $x_{i}$, of degree $\leq n$. Combined with the POLRAT in the front of (David) (whose degree in $x_{i}$ is $\leq 2 k+1$, by the last sentence of the statement of $(s u b)^{3}$ lemma 1.4.1.2) we have a POLRAT in $x_{i}$ of degree $\leq n+2 k+1$ in $x_{i}$.

Hence,

$\operatorname{Res}_{x_{i}, x_{\pi(v+1)}, \ldots, x_{\pi(k)}}\left[\frac{\widetilde{B}_{i}\left(x_{i} \rightarrow \bar{x}_{i}\right)}{1-x_{i} x_{R}}\right]=\operatorname{Res}_{x_{i}, x_{\pi(v+1)}, \ldots, x_{\pi(u-1)}}\left[\frac{\operatorname{POLRAT}\left(x_{i} ; x_{\pi(1)}, \ldots, \widehat{x}_{i}, \ldots, x_{\pi(u-1)}\right)}{x_{i}^{n+i+k+1} \bar{x}_{i}^{n+1}}\right]$,

$\left(\right.$ David $\left.^{\prime}\right)$ 
where the POLRAT in (David') has degree $\leq n+2 k+1$ in $x_{i}$. Applying $\operatorname{Res}_{x_{\pi(v+1)}, \ldots, x_{\pi(u-1)}}$ to the residuand of the right side of $\left(\right.$ David $\left.^{\prime}\right)$ gets rid of the dependence on $\left(x_{\pi(v+1)}, \ldots, x_{\pi(u-1)}\right)$, so we have:

$$
\operatorname{Res}_{x_{i}, x_{\pi(v+1)}, \ldots, x_{\pi(k)}}\left[\frac{\widetilde{B}_{i}\left(x_{i} \rightarrow \bar{x}_{i}\right)}{1-x_{i} x_{R}}\right]=\operatorname{Res}_{x_{i}}\left[\frac{\operatorname{POLRAT}\left(x_{i} ; x_{\pi(1)}, \ldots, x_{\pi(v-1)}\right)}{x_{i}^{n+i+k+1} \bar{x}_{i}^{n+1}}\right] \quad, \quad\left(\text { David }^{\prime \prime}\right)
$$

where $\operatorname{POLRAT}\left(x_{i} ; x_{\pi(1)}, \ldots, x_{\pi(v-1)}\right)$ has degree $\leq n+2 k+1$ in $x_{i}$.

But, the right of (David") can be rewritten as:

$$
\operatorname{Res}_{x_{i}}\left[\frac{\bar{x}_{i}^{i+k} \cdot \operatorname{POLRAT}\left(x_{i} ; x_{\pi(1)}, \ldots, x_{\pi(v-1)}\right)}{x_{i}^{n+i+k+1} \bar{x}_{i}^{n+i+k+1}}\right] .
$$

By crucial fact $\aleph_{5}^{\prime}$, since $(i+k)+n+(2 k+1) \leq 2(n+i+k)$ (recall that always $n \geq k$ and $\left.i \geq 1\right)$, we can perform $x_{i} \rightarrow \bar{x}_{i}$ in the residuand of (David'"'), so

$$
\operatorname{Res}_{x_{i}, x_{\pi(v+1)}, \ldots, x_{\pi(k)}}\left[\frac{\widetilde{B}_{i}\left(x_{i} \rightarrow \bar{x}_{i}\right)}{1-x_{i} x_{R}}\right]=\operatorname{Res}_{x_{i}, x_{\pi(v+1)}, \ldots, x_{\pi(k)}}\left[\frac{\widetilde{B}_{i}}{1-\bar{x}_{i} x_{R}}\right] .
$$

Applying $\operatorname{Res}_{x_{\pi(1)}, \ldots, x_{\pi(v-1)}}$ to both sides of the above equation finishes up the proof of (Michal). As we said, the proof of $\left(M i c h a l^{\prime}\right)$ is left to the reader. This finishes the proof of $(s u b)^{4}$ lemma 1.4.1.5.1.

Going back to the proof of case II of $(s u b)^{3}$ lemma 1.4.1.5, we have to prove that the right sides of $\left(\right.$ Michal) and $\left(\right.$ Michal $\left.^{\prime}\right)$ are equal to each other. By the above $(s u b)^{4}$ lemma, this would follow from their left sides being equal to each other. But the proof of this is exactly as that of case II of $(s u b)^{3}$ lemma 1.4.1.4: one proves the analog of $($ Noam $)$ and $(\overline{\text { Noam }})$, which reduce the statements to that of subcase Ia. This completes the proof of $(s u b)^{3}$ lemma 1.4.1.5.

We have one more $(s u b)^{3}$ lemma to go before we can complete the proof of $(s u b)^{2}$ lemma 1.4.1, which is to show that the last sum on the right side of (Celia) (in the statement of $(s u b)^{3}$ lemma 1.4.1.2, also has property (Hadas).)

Subsubsublemma 1.4.1.6: Let $\widetilde{B}_{i},(R<i \leq k)$, be as in the statement of 1.4.1.2, i.e. given by $(\widetilde{B} i 2)$. Then

$$
\operatorname{Res}_{x_{\pi(1)}, \ldots, x_{\pi(k)}}\left[\frac{\widetilde{B}_{i}}{1-z_{i} \bar{x}_{R}}\right]=\operatorname{Res}_{x_{\pi(1)}, \ldots, x_{\pi(k)}}\left[\frac{\widetilde{B}_{i}}{1-z_{i} \bar{x}_{R}}\left(x_{R} \rightarrow \bar{x}_{R}\right)\right] .
$$

[ Type 'S1416(k,n):' in ROBBINS, for specific $k$ and $n$.

Proof: The form of $\widetilde{B}_{i}$, for $R<i \leq k$, given by $(\widetilde{B} i 2)$ is identical to that of $\widetilde{A}_{i}$, for $R<i \leq k$, given by $(\widetilde{A} i 2)$. The only difference between the statement of $(s u b)^{3}$ lemma 1.4.1.4 and that of 1.4.1.6 is 
that the $x_{R}$ in the denominator of $\frac{\widetilde{A}_{i}}{1-z_{i} x_{R}}$ is changed into $\bar{x}_{R}: \frac{\widetilde{B}_{i}}{1-z_{i} \bar{x}_{R}}$. The proof goes verbatim. This completes the proof of $(s u b)^{3}$ lemma 1.4.1.6.

Going back to the proof of $(s u b)^{2}$ lemma 1.4.1, we know by $(s u b)^{3}$ lemmas $(1.4 .1 .3-6)$ that each part of $($ Celia $)$, in the statement of $(s u b)^{3}$ lemma 1.4.1.2, has property (Hadas), and by the additivity of this property, so does the whole $F_{n, k}\left(\varepsilon_{1}\left(x_{1}\right), \ldots, x_{R}, \ldots, \varepsilon_{k}\left(x_{k}\right)\right)$. But for the latter to have property (Hadas) is precisely what $(s u b)^{2}$ lemma 1.4 .1 is saying. This completes the proof of $(s u b)^{2}$ lemma 1.4.1.

As we noted at the very beginning of the proof of sublemma 1.4, subsublemma 1.4.1, read from right to left, can be used repeatedly to 'unbar' $x_{i}$ 's, until we get that

$$
\operatorname{Res}_{x_{\pi(1)}, \ldots, x_{\pi(k)}}\left[F_{n, k}\left(\varepsilon_{1}\left(x_{1}\right), \ldots, \varepsilon_{k}\left(x_{k}\right)\right)\right]=\operatorname{Res}_{x_{\pi(1)}, \ldots, x_{\pi(k)}}\left[F_{n, k}\left(x_{1}, \ldots, x_{k}\right)\right] \quad . \quad \text { (SofTov) }
$$

Since $F_{n, k}\left(x_{1}, \ldots, x_{k}\right)$ has a genuine Laurent expansion, crucial fact $\aleph_{4}^{\prime}$ reassures us that we can unscramble the order of residue-taking on the right side of (SofTov):

$$
\operatorname{Res}_{x_{\pi(1)}, \ldots, x_{\pi(k)}}\left[F_{n, k}\left(x_{1}, \ldots, x_{k}\right)\right]=\operatorname{Res}_{x_{1}, \ldots, x_{k}}\left[F_{n, k}\left(x_{1}, \ldots, x_{k}\right)\right]
$$

Combining (SofTov) with (HakolTov) yields $\left(1.4^{\prime}\right)$, which we noted was equivalent to the statement of sublemma 1.4. This completes the proof of sublemma 1.4.

\section{INTERACT, Part II}

At this juncture, we have to introduce the rational function $T_{k, n}(x)=T_{k, n}\left(x_{1}, \ldots, x_{k}\right)$ defined by:

$$
T_{k, n}(x):=\prod_{i=1}^{k} \frac{1}{\left(\bar{x}_{i} x_{i}\right)^{n+k+1}} \prod_{1 \leq i<j \leq k} \frac{1}{\left(1-x_{i} x_{j}\right)\left(1-\bar{x}_{i} x_{j}\right)\left(1-x_{i} \bar{x}_{j}\right)\left(1-\bar{x}_{i} \bar{x}_{j}\right)} .
$$

We can rewrite Sublemmas 1.1' and 1.2' more compactly as follows.

Sublemma 1.1" The total number of $n \times k$-Magog trapezoids, $b_{k}(n)$, is given by:

$$
\operatorname{Res}_{x_{1}, \ldots, x_{k}}\left[\Delta_{k}(x) T_{k, n}(x) \prod_{i=1}^{k} x_{i}^{i+1} \prod_{1 \leq i<j \leq k}\left(1-\bar{x}_{i} x_{j}\right)\left(1-x_{i} \bar{x}_{j}\right)\left(1-\bar{x}_{i} \bar{x}_{j}\right)\right] \quad \cdot\left(\text { MagogTotal }^{\prime \prime}\right)
$$

\section{Proof:}

Sublemma 1.2": The total number of $n \times k-$ Gog trapezoids, $m_{k}(n)$, is given by:

$$
\left.\operatorname{Res}_{x_{1}, \ldots, x_{k}}\left[\Phi_{k}(x) T_{k, n}(x) \prod_{i=1}^{k}\left(\bar{x}_{i}\right)^{k-i} x_{i}^{k} \prod_{1 \leq i<j \leq k}\left(1-x_{i} \bar{x}_{j}\right)\left(1-\bar{x}_{i} \bar{x}_{j}\right)\right] \quad, \quad \quad \text { (GogTotal }{ }^{\prime \prime}\right)
$$


where $\Phi_{k}(x)=\Phi_{k}\left(x_{1}, \ldots, x_{k}\right)$ is the polynomial defined in $\left(G_{o g}\right)$.

\section{Proof:}

Using sublemmas 1.3 and 1.4, and the fact that $T_{k, n}(x)$ is symmetric while $\Delta_{k}$ and $\Phi_{k}$ are antisymmetric with respect to the action of the group of signed permutations $W\left(\mathcal{B}_{k}\right)$, we can average Sublemmas 1.1" and 1.2", to get yet other residue expressions for the desired quantities $b_{k}(n)$ and $m_{k}(n)$, as follows.

Sublemma 1.1"': The total number of $n \times k$-Magog trapezoids, $b_{k}(n)$, is given by:

$\frac{1}{2^{k} k !} \operatorname{Res}_{x_{1}, \ldots, x_{k}}\left\{T_{k, n}(x) \Delta_{k}(x) \cdot \sum_{g \in W\left(\mathcal{B}_{k}\right)} \operatorname{sgn}(g) \cdot g\left[\prod_{i=1}^{k} x_{i}^{i+1} \prod_{1 \leq i<j \leq k}\left(1-\bar{x}_{i} x_{j}\right)\left(1-x_{i} \bar{x}_{j}\right)\left(1-\bar{x}_{i} \bar{x}_{j}\right)\right]\right\}$

Proof:

Sublemma 1.2"': The total number of $n \times k$-Gog trapezoids, $m_{k}(n)$, is given by ,

$$
\frac{(-1)^{k}}{2^{k} k !} \operatorname{Res}_{x_{1}, \ldots, x_{k}}\left[T_{k, n}(x) \Phi_{k}(x)^{2}\right] \quad,
$$

where $\Phi_{k}(x)=\Phi_{k}\left(x_{1}, \ldots, x_{k}\right)$ is the polynomial defined by $\left(\operatorname{Gog}_{1}\right)$, in the statement of sublemma 1.2 .

Proof: See the definition of $\Phi_{k}(x)$ given in $\left(\operatorname{Gog}_{1}\right) \square$. 


\section{Act V. GOG $=$ MAGOG}

... Gog and Magog, to gather them together to battle: the number of whom is as the sand of the sea. - $\quad$ (Revelations XX,8)

We have one more sublemma to go, that asserts that not only are the iterated-residues expressing $b_{k}(n)$ and $m_{k}(n)$, as given in $\left(\right.$ MagogTotal $\left.^{\prime \prime \prime}\right)$ and $\left(\right.$ GogTotal $\left.^{\prime \prime \prime}\right)$ respectively, equal to each other, but the actual residue-ands are.

Sublemma 1.5: Let $\Phi_{k}(x)=\Phi_{k}\left(x_{1}, \ldots, x_{k}\right)$ and $\Delta_{k}(x)=\Delta_{k}\left(x_{1}, \ldots, x_{k}\right)$ be defined as above, i.e. by

$$
\begin{gathered}
\Phi_{k}\left(x_{1}, \ldots, x_{k}\right):=(-1)^{k} \sum_{g \in W\left(\mathcal{B}_{k}\right)} \operatorname{sgn}(g) \cdot g\left[\prod_{i=1}^{k} \bar{x}_{i}^{k-i} x_{i}^{k} \prod_{1 \leq i<j \leq k}\left(1-x_{i} \bar{x}_{j}\right)\left(1-\bar{x}_{i} \bar{x}_{j}\right)\right] \\
\Delta_{k}\left(x_{1}, \ldots, x_{k}\right):=\prod_{i=1}^{k}\left(1-2 x_{i}\right) \prod_{1 \leq i<j \leq k}\left(x_{j}-x_{i}\right)\left(x_{j}+x_{i}-1\right)
\end{gathered}
$$

The following identity holds:

$\Delta_{k}\left(x_{1}, \ldots, x_{k}\right) \sum_{g \in W\left(\mathcal{B}_{k}\right)} \operatorname{sgn}(g) \cdot g\left[\prod_{i=1}^{k} x_{i}^{i+1} \prod_{1 \leq i<j \leq k}\left(1-\bar{x}_{i} x_{j}\right)\left(1-x_{i} \bar{x}_{j}\right)\left(1-\bar{x}_{i} \bar{x}_{j}\right)\right]=\begin{gathered}(-1)^{k} \Phi_{k}\left(x_{1}, \ldots, x_{k}\right)^{2} \\ (\text { Gog }=\text { Magog })\end{gathered}$

[ Type 'S15(k):' in ROBBINS, for specific k.]

Proof: Since $\Phi_{k}(x)$ is $W\left(\mathcal{B}_{k}\right)$-antisymmetric, it can be written as $\Phi_{k}(x)=\Delta_{k}(x) \Omega_{k}(x)$, for some polynomial $\Omega_{k}(x)=\Omega_{k}\left(x_{1}, \ldots, x_{k}\right)$ that is symmetric with respect to $W\left(\mathcal{B}_{k}\right)$. This means that $\Omega_{k}(x)$ is a symmetric polynomial in $\left(x_{1} \bar{x}_{1}, \ldots, x_{k} \bar{x}_{k}\right)$. Since the degree of $\Phi_{k}(x)$, viewed as a polynomial in $x_{1}$, is $\leq(2 k-1)+2(k-1)$, and that of $\Delta_{k}(x)$ is $1+2(k-1)$, it follows that the degree of $\Omega_{k}(x)$, as a polynomial in $x_{1}$ is $\leq 2 k-2$, and hence as a polynomial in $x_{1} \bar{x}_{1}$ is $\leq k-1$. Similarly, the left side of (Gog=Magog) can be written as $(-1)^{k} \Delta_{k}(x)^{2} L_{k}(x)$, for some polynomial $L_{k}(x)$, that is symmetric in $\left(x_{1} \bar{x}_{1}, \ldots, x_{k} \bar{x}_{k}\right)$. The degree of $L_{k}$, as a polynomial in $x_{1}$, is $\leq(k+1)+3(k-1)-(1+2(k-1))=2 k-1$, and hence its degree in $x_{1} \bar{x}_{1}$ is $\leq k-1$. We have to prove that $L_{k}-\Omega_{k}^{2}$ is identically zero. Since $L_{k}-\Omega_{k}^{2}$ is a polynomial of degree $\leq 2 k-2$ in $x_{1} \bar{x}_{1}$, it suffices to prove that it is zero at $2 k-1$ distinct values of $x_{1} \bar{x}_{1}$. This will follow by induction on $k$ from the following two subsublemmas.

Subsublemma 1.5.1: Let $L_{k}(x)$ be the left side of (Gog=Magog) divided by $\Delta_{k}(x)^{2}$. We have

$$
\left.L_{k}\left(x_{1}, \ldots, x_{k}\right)\right|_{x_{1}=x_{i}^{-1}}=\left(\prod_{\substack{j=2 \\ j \neq i}}^{k} \frac{\left(1-\bar{x}_{i} x_{j}\right)\left(1-\bar{x}_{i} \bar{x}_{j}\right)}{x_{i}}\right)^{2} L_{k-2}\left(x_{2}, \ldots, \widehat{x}_{i}, \ldots, x_{k}\right) \quad, \quad(i=2, \ldots, k)
$$




$$
\begin{gathered}
\left.L_{k}\left(x_{1}, \ldots, x_{k}\right)\right|_{x_{1}=\bar{x}_{i}^{-1}}=\left(\prod_{\substack{j=2 \\
j \neq i}}^{k} \frac{\left(1-x_{i} x_{j}\right)\left(1-x_{i} \bar{x}_{j}\right)}{\bar{x}_{i}}\right)^{2} L_{k-2}\left(x_{2}, \ldots, \widehat{x}_{i}, \ldots, x_{k}\right) \quad, \quad(i=2, \ldots, k) \\
\left.L_{k}\left(x_{1}, \ldots, x_{k}\right)\right|_{x_{1}=0}=L_{k-1}\left(x_{2}, \ldots, x_{k}\right) .
\end{gathered}
$$

[ Type 'S151(k):' in ROBBINS, for specific k.]

Proof: By definition, $L_{k}(x)$ is the left side of (Gog=Magog) divided by $\Delta_{k}(x)^{2}$. By using the definition $\left(\right.$ Delta) of $\Delta_{k}(x)$, as well as its $W\left(\mathcal{B}_{k}\right)$-antisymmetry, we get,

$$
\begin{gathered}
L_{k}\left(x_{1}, \ldots, x_{k}\right)=\sum_{g \in W\left(\mathcal{B}_{k}\right)} g\left[\prod_{i=1}^{k} \frac{x_{i}^{i+1}}{\left(1-2 x_{i}\right)} \prod_{1 \leq i<j \leq k} \frac{\left(1-\bar{x}_{i} x_{j}\right)\left(1-x_{i} \bar{x}_{j}\right)\left(1-\bar{x}_{i} \bar{x}_{j}\right)}{\left(x_{j}-x_{i}\right)\left(x_{j}+x_{i}-1\right)}=\right. \\
\sum_{\varepsilon \in\{+1,-1\}^{k}} \varepsilon\left\{\sum_{\pi \in \mathcal{S}_{k}} \pi\left[\prod_{i=1}^{k} \frac{x_{i}^{i+1}}{\left(1-2 x_{i}\right)} \prod_{1 \leq i<j \leq k} \frac{\left(1-\bar{x}_{i} x_{j}\right)\left(1-x_{i} \bar{x}_{j}\right)\left(1-\bar{x}_{i} \bar{x}_{j}\right)}{\left(x_{j}-x_{i}\right)\left(x_{j}+x_{i}-1\right)}\right]\right\} .
\end{gathered}
$$

Since the argument of $\pi$ is antisymmetric w.r.t. to $\mathcal{S}_{k}$, except for $\prod_{i=1}^{k} x_{i}^{i+1}=\prod_{i=1}^{k} x_{i}^{2} \prod_{i=1}^{k} x_{i}^{i-1}$, it follows, by Vandermonde's determinant identity (see "Crucial Facts"), that

$$
L_{k}\left(x_{1}, \ldots, x_{k}\right)=\sum_{\varepsilon \in\{+1,-1\}^{k}} \varepsilon\left\{\prod_{i=1}^{k} \frac{x_{i}^{2}}{\left(1-2 x_{i}\right)} \prod_{1 \leq i<j \leq k} \frac{\left(1-\bar{x}_{i} x_{j}\right)\left(1-x_{i} \bar{x}_{j}\right)\left(1-\bar{x}_{i} \bar{x}_{j}\right)}{\left(x_{j}+x_{i}-1\right)}\right\} .
$$

To prove (1.5.1a) for $i=2, \ldots, k$, it suffices, by symmetry, to consider $i=2$. This would also imply, by the symmetry $x \leftrightarrow \bar{x}$, built into $W\left(\mathcal{B}_{k}\right)$, that $(1.5 .1 \mathrm{~b})$ is true. So let's plug in $x_{1}=x_{2}^{-1}$ in (1.5.1d). The only way that $\varepsilon\left[\left(1-\bar{x}_{1} x_{2}\right)\left(1-x_{1} \bar{x}_{2}\right)\left(1-\bar{x}_{1} \bar{x}_{2}\right)\right]$ cannot be zero then, is for $\varepsilon_{1}$ and $\varepsilon_{2}$ to be both +1 . Hence the $2^{k}$-summand sum (1.5.1d) shrinks to a $2^{k-2}$-summand sum:

$$
\begin{aligned}
& \frac{\left(x_{2}^{-1}\right)^{2}}{\left(1-2 x_{2}^{-1}\right)} \frac{\left(x_{2}\right)^{2}}{\left(1-2 x_{2}\right)} \frac{\left(1-\overline{x_{2}^{-1}} x_{2}\right)\left(1-x_{2}^{-1} \bar{x}_{2}\right)\left(1-\overline{x_{2}^{-1}} \bar{x}_{2}\right)}{x_{2}+x_{2}^{-1}-1} \sum_{\substack{\varepsilon \in\{+1,-1\} k \\
\varepsilon_{1}=1, \varepsilon_{2}=1}} \varepsilon\left[\prod _ { j = 3 } ^ { k } \left(\frac{\left(1-\overline{x_{2}^{-1}} x_{j}\right)\left(1-x_{2}^{-1} \bar{x}_{j}\right)\left(1-\bar{x}_{2}^{-1} \bar{x}_{j}\right)}{x_{j}+x_{2}^{-1}-1} .\right.\right. \\
& \left.\left.\frac{\left(1-\bar{x}_{2} x_{j}\right)\left(1-x_{2} \bar{x}_{j}\right)\left(1-\bar{x}_{2} \bar{x}_{j}\right)}{x_{j}+x_{2}-1}\right) \prod_{j=3}^{k} \frac{x_{j}^{2}}{1-2 x_{j}} \prod_{3 \leq i<j \leq k} \frac{\left(1-\bar{x}_{i} x_{j}\right)\left(1-x_{i} \bar{x}_{j}\right)\left(1-\bar{x}_{i} \bar{x}_{j}\right)}{\left(x_{j}+x_{i}-1\right)}\right] . \quad \text { (1.5.1e) }
\end{aligned}
$$

We now need the following utterly trivial subsubsublemmas.

\section{Subsubsublemma 1.5.1.1:}

$$
\frac{\left(1-\overline{x_{2}^{-1}} t\right)\left(1-x_{2}^{-1} \bar{t}\right)\left(1-\overline{x_{2}^{-1}} \bar{t}\right)}{t+x_{2}^{-1}-1} \cdot \frac{\left(1-\overline{x_{2}} t\right)\left(1-x_{2} \bar{t}\right)\left(1-\bar{x}_{2} \bar{t}\right)}{t+x_{2}-1}=\left(\frac{\left(1-\bar{x}_{2} \bar{t}\right)\left(1-\bar{x}_{2} t\right)}{x_{2}}\right)^{2} .
$$


[ Type 'S1511():' in ROBBINS, to get a rigorous proof.]

Proof:

Subsubsublemma 1.5.1.2:

$$
\frac{\left(x_{2}^{-1}\right)^{2}}{\left(1-2 x_{2}^{-1}\right)} \frac{\left(x_{2}\right)^{2}}{\left(1-2 x_{2}\right)} \frac{\left(1-\overline{x_{2}^{-1}} x_{2}\right)\left(1-x_{2}^{-1} \bar{x}_{2}\right)\left(1-\overline{x_{2}^{-1}} \bar{x}_{2}\right)}{x_{2}+x_{2}^{-1}-1}=1
$$

[ Type 'S1512() :' in ROBBINS, to get a rigorous proof.]

\section{Proof: $\square$.}

Substituting (1.5.1.1) and (1.5.1.2) into (1.5.1e) yields:

$$
\begin{gathered}
L_{k}\left(x_{2}^{-1}, x_{2}, x_{3}, \ldots, x_{k}\right)=\sum_{\substack{\varepsilon \in\{+1,-1\} k \\
\varepsilon_{1}=1, \varepsilon_{2}=1}} \varepsilon\left[\prod_{j=3}^{k}\left(\frac{\left(1-\bar{x}_{2} \bar{x}_{j}\right)\left(1-\bar{x}_{2} x_{j}\right)}{x_{2}}\right)^{2} \cdot\right. \\
\left.\prod_{j=3}^{k} \frac{x_{j}^{2}}{2 x_{j}-1} \prod_{3 \leq i<j \leq k} \frac{\left(1-\bar{x}_{i} x_{j}\right)\left(1-x_{i} \bar{x}_{j}\right)\left(1-\bar{x}_{i} \bar{x}_{j}\right)}{\left(x_{j}+x_{i}-1\right)}\right]=\left(\prod_{j=3}^{k} \frac{\left(1-\bar{x}_{2} \bar{x}_{j}\right)\left(1-\bar{x}_{2} x_{j}\right)}{x_{2}}\right)^{2} L_{k-2}\left(x_{3}, \ldots, x_{k}\right) .
\end{gathered}
$$

This completes the proof of (1.5.1a) and (1.5.1b). To prove (1.5.1c), note that when $x_{1}=0$, the only non-zero summands of (1.5.1d) are those for which $\varepsilon_{1}=-1$. The $2^{k}$-summand sum (1.5.1d) collapses then to a $2^{k-1}$-summand sum as follows:

$$
\sum_{\substack{\varepsilon \in\{+1,-1\} k \\ \varepsilon_{1}=-1}} \varepsilon\left(\prod_{i=2}^{k} \frac{x_{i}^{2}}{1-2 x_{i}} \prod_{j=2}^{k} \frac{\left(1-\bar{x}_{j}\right)}{x_{j}} \prod_{2 \leq i<j \leq k} \frac{\left(1-\bar{x}_{i} x_{j}\right)\left(1-x_{i} \bar{x}_{j}\right)\left(1-\bar{x}_{i} \bar{x}_{j}\right)}{\left(x_{j}+x_{i}-1\right)}\right)=L_{k-1}\left(x_{2}, \ldots, x_{k}\right)
$$

This completes the proof of subsublemma 1.5.1 $\square$.

Subsublemma 1.5.2: $\Omega_{k}(x):=\Phi_{k}(x) / \Delta_{k}(x)$ (see $\left(G_{1} g_{1}\right)$ and (Delta), in the statement of sublemma 1.5 for definitions), satisfies

$$
\begin{aligned}
&\left.\Omega_{k}\left(x_{1}, \ldots, x_{k}\right)\right|_{x_{1}=x_{i}^{-1}}=\left(\prod_{\substack{j=2 \\
j \neq i}}^{k} \frac{\left(1-\bar{x}_{i} x_{j}\right)\left(1-\bar{x}_{i} \bar{x}_{j}\right)}{x_{i}}\right) \Omega_{k-2}\left(x_{2}, \ldots, \widehat{x}_{i}, \ldots, x_{k}\right) \quad, \quad(i=2, \ldots, k) \\
&\left.\Omega_{k}\left(x_{1}, \ldots, x_{k}\right)\right|_{x_{1}=\bar{x}_{i}^{-1}}=\left(\prod_{\substack{j=2 \\
j \neq i}}^{k} \frac{\left(1-x_{i} x_{j}\right)\left(1-x_{i} \bar{x}_{j}\right)}{\bar{x}_{i}}\right) \Omega_{k-2}\left(x_{2}, \ldots, \widehat{x}_{i}, \ldots, x_{k}\right) \quad, \quad(i=2, \ldots, k) \\
&\left.\Omega_{k}\left(x_{1}, \ldots, x_{k}\right)\right|_{x_{1}=0}=\Omega_{k-1}\left(x_{2}, \ldots, x_{k}\right) \quad .(1.5 .2 b) \\
&(1.5 .2 c)
\end{aligned}
$$

[ Type 'S152(k):' in ROBBINS, for specific k.] 
Proof: By $W\left(\mathcal{B}_{k}\right)$ symmetry, it is enough to prove $(1.5 .2 \mathrm{a})$ for $x_{1}=x_{2}^{-1}$, which also implies (1.5.2b). To wit, we have to prove that:

$$
\left.x_{2}^{k-2} \Omega_{k}\left(x_{1}, \ldots, x_{k}\right)\right|_{x_{1}=x_{2}^{-1}}=\left(\prod_{j=3}^{k}\left(1-\bar{x}_{2} x_{j}\right)\left(1-\bar{x}_{2} \bar{x}_{j}\right)\right) \Omega_{k-2}\left(x_{3}, \ldots, x_{k}\right) .
$$

We now need:

Subsubsublemma 1.5.2.1: Let $\Omega_{k}(x):=\Phi_{k}(x) / \Delta_{k}(x)$ be as in the statement of subsublemma 1.5.2, then

$$
\left.\Omega_{k}\left(x_{1}, \ldots, x_{k}\right)\right|_{x_{1}=x_{2}^{-1}}
$$

is a Laurent polynomial in $x_{2}$ of degree $\leq(k-2)$ and of low-degree $\geq-(k-2)$.

[The low-degree, in $x$, of a Laurent polynomial $f(x)$ is the degree of $f\left(x^{-1}\right)$.]

[ Type 'S1521(k):' in ROBBINS, for specific k.]

Proof: It is readily checked that $\left.\Delta_{k}\left(x_{1}, \ldots, x_{k}\right)\right|_{x_{1}=x_{2}^{-1}}$ is a Laurent polynomial in $x_{2}$ of degree $(2 k-1)$ and low-degree $-(2 k-1)$, the statement of the $(s u b)^{3}$ lemma is equivalent to the statement that $\left.\Phi_{k}\left(x_{1}, \ldots, x_{k}\right)\right|_{x_{1}=x_{2}^{-1}}$ is a Laurent polynomial in $x_{2}$ of degree $\leq 3 k-3$ and low-degree $\geq$ $-(3 k-3)$. We will prove that this is even true for each and every single term in the defining sum $\left(\operatorname{Gog}_{1}\right)$ of $\Phi_{k}$. Let $g=(\pi, \varepsilon)$ be a typical signed permutation. Let $\alpha:=\pi^{-1}(1)$ and $\beta:=\pi^{-1}(2)$, so that $\pi(\alpha)=1$ and $\pi(\beta)=2$. Without loss of generality we can assume that $\alpha<\beta$, since in the opposite case we can exchange $x_{1}$ and $x_{2}$. Let $\operatorname{POL}\left(\widehat{x}_{1}, \widehat{x}_{2}\right)$ denote a polynomial in $\left(x_{3}, \ldots, x_{k}\right)$. Let $z_{i}:=\varepsilon_{i}\left(x_{i}\right)$ (i.e. $z_{i}=x_{i}$ if $\varepsilon_{i}=1$ and $z_{i}=\bar{x}_{i}\left(=1-x_{i}\right)$ if $\varepsilon_{i}=-1$.)

The typical term corresponding to $g=(\pi, \varepsilon)$ in the definition $\left(G o g_{1}\right)$ of $\Phi_{k}$ can be written as follows:

$$
\begin{aligned}
g\left[\prod_{i=1}^{k} \bar{x}_{i}^{k-i} x_{i}^{k} \prod_{1 \leq i<j \leq k}\left(1-x_{i} \bar{x}_{j}\right)\left(1-\bar{x}_{i} \bar{x}_{j}\right)\right]=\prod_{i=1}^{k} \bar{z}_{\pi(i)}^{k-i} z_{\pi(i)}^{k} \prod_{1 \leq i<j \leq k}\left(1-z_{\pi(i)} \bar{z}_{\pi(j)}\right)\left(1-\bar{z}_{\pi(i)} \bar{z}_{\pi(j)}\right) \\
=\operatorname{POL}\left(\widehat{x}_{1}, \widehat{x}_{2}\right) \cdot\left(\bar{z}_{1}^{k-\alpha} z_{1}^{k}\right) \cdot\left(\bar{z}_{2}^{k-\beta} z_{2}^{k}\right)\left(1-z_{1} \bar{z}_{2}\right)\left(1-\bar{z}_{1} \bar{z}_{2}\right) \prod_{i=1}^{\alpha-1}\left(1-z_{\pi(i)} \bar{z}_{1}\right)\left(1-\bar{z}_{\pi(i)} \bar{z}_{1}\right) \prod_{\substack{j=\alpha+1 \\
j \neq \beta}}^{k}\left(1-z_{1} \bar{z}_{\pi(j)}\right)\left(1-\bar{z}_{1} \bar{z}_{\pi(j)}\right) \\
\\
\qquad \prod_{\substack{i=1 \\
i \neq \alpha}}^{\beta-1}\left(1-z_{\pi(i)} \bar{z}_{2}\right)\left(1-\bar{z}_{\pi(i)} \bar{z}_{2}\right) \prod_{j=\beta+1}^{k}\left(1-z_{2} \bar{z}_{\pi(j)}\right)\left(1-\bar{z}_{2} \bar{z}_{\pi(j)}\right)
\end{aligned}
$$

If $\varepsilon_{2}=-1$, i.e. $z_{2}=\bar{x}_{2}$, the above term must vanish when $x_{1} x_{2}=1$, because of $\left(1-z_{1} \bar{z}_{2}\right)\left(1-\bar{z}_{1} \bar{z}_{2}\right)$. So we can assume that $\varepsilon_{2}=+1$, and so $z_{2}=x_{2}$. We now distinguish two cases:

Case I: $\varepsilon_{1}=+1$, i.e. $z_{1}=x_{1}$, then the term is

$\operatorname{POL}\left(\widehat{x}_{1}, \widehat{x}_{2}\right) \cdot\left(\bar{x}_{1}^{k-\alpha} x_{1}^{k}\right) \cdot\left(\bar{x}_{2}^{k-\beta} x_{2}^{k}\right)\left(1-x_{1} \bar{x}_{2}\right)\left(1-\bar{x}_{1} \bar{x}_{2}\right) \prod_{i=1}^{\alpha-1}\left(1-z_{\pi(i)} \bar{x}_{1}\right)\left(1-\bar{z}_{\pi(i)} \bar{x}_{1}\right) \prod_{\substack{j=\alpha+1 \\ j \neq \beta}}^{k}\left(1-x_{1} \bar{z}_{\pi(j)}\right)\left(1-\bar{x}_{1} \bar{z}_{\pi(j)}\right)$. 


$$
\prod_{\substack{i=1 \\ i \neq \alpha}}^{\beta-1}\left(1-z_{\pi(i)} \bar{x}_{2}\right)\left(1-\bar{z}_{\pi(i)} \bar{x}_{2}\right) \prod_{j=\beta+1}^{k}\left(1-x_{2} \bar{z}_{\pi(j)}\right)\left(1-\bar{x}_{2} \bar{z}_{\pi(j)}\right)
$$

Now plug in $x_{1}=x_{2}^{-1}$. The product of the four products above times $\left(1-x_{2}^{-1} \bar{x}_{2}\right)\left(1-\overline{x_{2}^{-1}} \bar{x}_{2}\right)$ $\left(\left(1-x_{1} \bar{x}_{2}\right)\left(1-\bar{x}_{1} \bar{x}_{2}\right)\right.$ evaluated at $\left.x_{1}=x_{2}^{-1}\right)$ is a Laurent polynomial in $x_{2}$ of degree $\leq 2(k-2)+2=$ $2 k-2$ and low-degree $\geq-(2 k-2)$. On the other hand $\left(1-x_{2}^{-1}\right)^{k-\alpha}\left(1-x_{2}\right)^{k-\beta}$ has degree $\leq k-\beta \leq k-1$ and low-degree $\geq-(k-\alpha) \geq-(k-1)$. So the degree in $x_{2}$ of the whole term is $\leq 2 k-2+k-1 \leq 3 k-3$, and its low-degree is $\geq-[(2 k-2)+k-1] \geq-(3 k-3)$. This completes case I of subsubsublemma 1.5.2.1.

Case II: $\varepsilon_{1}=-1$, i.e. $z_{1}=\bar{x}_{1}$, then the term is

$$
\begin{gathered}
\operatorname{POL}\left(\widehat{x}_{1}, \widehat{x}_{2}\right) \cdot\left(x_{1}^{k-\alpha} \bar{x}_{1}^{k}\right) \cdot\left(\bar{x}_{2}^{k-\beta} x_{2}^{k}\right)\left(1-\bar{x}_{1} \bar{x}_{2}\right)\left(1-x_{1} \bar{x}_{2}\right) \prod_{i=1}^{\alpha-1}\left(1-z_{\pi(i)} x_{1}\right)\left(1-\bar{z}_{\pi(i)} x_{1}\right) \prod_{\substack{j=\alpha+1 \\
j \neq \beta}}^{k}\left(1-\bar{x}_{1} \bar{z}_{\pi(j)}\right)\left(1-x_{1} \bar{z}_{\pi(j)}\right) \cdot \\
\prod_{\substack{i=1 \\
i \neq \alpha}}^{\beta-1}\left(1-z_{\pi(i)} \bar{x}_{2}\right)\left(1-\bar{z}_{\pi(i)} \bar{x}_{2}\right) \prod_{j=\beta+1}^{k}\left(1-x_{2} \bar{z}_{\pi(j)}\right)\left(1-\bar{x}_{2} \bar{z}_{\pi(j)}\right)
\end{gathered}
$$

Plugging in $x_{1}=x_{2}^{-1}$ above yields a Laurent polynomial in $x_{2}$ of degree $\leq 2(k-2)+2=2 k-2$ and low-degree $\geq-(2 k-2)$, times

$$
\left(x_{1}^{k-\alpha} \bar{x}_{1}^{k}\right) \cdot\left(\bar{x}_{2}^{k-\beta} x_{2}^{k}\right)=\left(x_{1} x_{2}\right)^{k-\alpha} \bar{x}_{1}^{k} \bar{x}_{2}^{k-\beta} x_{2}^{\alpha}=\left(1-x_{2}^{-1}\right)^{k}\left(1-x_{2}\right)^{k-\beta} x_{2}^{\alpha} .
$$

But $\left(1-x_{2}^{-1}\right)^{k}\left(1-x_{2}\right)^{k-\beta} x_{2}^{\alpha}$ has degree $\leq k-\beta+\alpha \leq k-1$ and low-degree $\geq-(k-\alpha) \geq-(k-1)$. So the degree in $x_{2}$ of the whole term is $\leq 2 k-2+k-1 \leq 3 k-3$, and its low-degree is $\geq-[(2 k-2)+k-1] \geq-(3 k-3)$. This completes the proof of subsubsublemma 1.5.2.1.

Going back to the proof of subsublemma 1.5.2, both sides of (Dominique) are polynomials of degree $\leq 2(k-2)$ in $\bar{x}_{2}$. (By subsubsublemma 1.5.2.1, The left side is a polynomial in $x_{2}$ of degree $\leq 2(k-2)$, and hence also in $\bar{x}_{2}$, since $\left.x_{2}=1-\bar{x}_{2}.\right)$ The right side vanishes at the $2(k-2)$ values $\bar{x}_{2}=x_{j}^{-1}, \bar{x}_{2}=\bar{x}_{j}{ }^{-1}, j=3, \ldots, k$. The next subsubsublemma asserts that this is true as well for the left side of (Dominique).

Subsubsublemma 1.5.2.2: The left side of (Dominique),

$$
\left.x_{2}^{k-2} \Omega_{k}\left(x_{1}, \ldots, x_{k}\right)\right|_{x_{1}=x_{2}^{-1}}
$$

when viewed as a polynomial in $\bar{x}_{2}$, vanishes at the $2(k-2)$ values $\bar{x}_{2}=x_{j}^{-1}, \bar{x}_{2}=\bar{x}_{j}^{-1}$, for $j=3, \ldots, k$.

[ Type 'S1522(k) :' in ROBBINS, for specific k>2.] 
Proof: By the $W\left(\mathcal{B}_{k}\right)$-symmetry of $\Omega_{k}\left(x_{1}, \ldots, x_{k}\right)$, it suffices to prove that the left side of (Dominique) vanishes when $\bar{x}_{2}=x_{3}^{-1}$. This is equivalent to proving that $\Omega_{k}\left(x_{1}, \ldots, x_{k}\right)$ vanishes whenever both $1-x_{1} x_{2}=0$ and $1-\bar{x}_{2} x_{3}=0$. Note that these two last conditions also imply that $1-\bar{x}_{1} \bar{x}_{3}=0$. Since $\Delta_{k}(x)$ does not vanish there, it suffices to prove that $\Phi_{k}(x)$ vanishes when these relations hold. Putting $z_{i}:=\varepsilon_{i}\left(x_{i}\right), \Phi_{k}\left(x_{1}, \ldots, x_{k}\right)$ can be written as:

$\Phi_{k}\left(x_{1}, \ldots, x_{k}\right)=(-1)^{k} \sum_{\varepsilon \in\{-1,+1\}^{k}} \sum_{\pi \in \mathcal{S}_{k}} \operatorname{sgn}(\varepsilon) \operatorname{sgn}(\pi) \prod_{i=1}^{k} \bar{z}_{\pi(i)}^{k-i} z_{\pi(i)}^{k} \prod_{1 \leq i<j \leq k}\left(1-z_{\pi(i)} \bar{z}_{\pi(j)}\right)\left(1-\bar{z}_{\pi(i)} \bar{z}_{\pi(j)}\right)$.

(Bruce)

We will prove that each and every term of (Bruce) vanishes when $1-x_{1} x_{2}=0$ and $1-\bar{x}_{2} x_{3}=0$. We now split the sum in (Bruce) as follows:

$$
\begin{aligned}
& \sum_{\varepsilon \in\{-1,+1\}^{k}} \sum_{1 \leq \alpha<\beta<\gamma \leq k} \sum_{\substack{\pi \in \mathcal{S}_{k} \\
\{\pi(\alpha), \pi(\beta), \pi(\gamma)\}=\{1,2,3\}}} \operatorname{sgn}(\varepsilon) \operatorname{sgn}(\pi) \prod_{i=1}^{k} \bar{z}_{\pi(i)}^{k-i} z_{\pi(i)}^{k} \prod_{\substack{1 \leq i<j \leq k \\
i, j \notin\{\alpha, \beta, \gamma\}}}\left(1-z_{\pi(i)} \bar{z}_{\pi(j)}\right)\left(1-\bar{z}_{\pi(i)} \bar{z}_{\pi(j)}\right) . \\
& \left(1-z_{\pi(\alpha)} \bar{z}_{\pi(\beta)}\right)\left(1-\bar{z}_{\pi(\alpha)} \bar{z}_{\pi(\beta)}\right)\left(1-z_{\pi(\alpha)} \bar{z}_{\pi(\gamma)}\right)\left(1-\bar{z}_{\pi(\alpha)} \bar{z}_{\pi(\gamma)}\right)\left(1-z_{\pi(\beta)} \bar{z}_{\pi(\gamma)}\right)\left(1-\bar{z}_{\pi(\beta)} \bar{z}_{\pi(\gamma)}\right)
\end{aligned}
$$

$\left(\right.$ Bruce $\left.^{\prime}\right)$

For each such term in $\left(\right.$ Bruce $\left.^{\prime}\right)$, let $\sigma \in \mathcal{S}_{3}$ be defined by $\sigma(1)=\pi(\alpha), \sigma(2)=\pi(\beta)$, and $\sigma(3)=\pi(\gamma)$. Also let $\varepsilon^{\prime}$ be the first three components of $\varepsilon$ : i.e. $\varepsilon^{\prime}(1)=\varepsilon(1), \varepsilon^{\prime}(2)=\varepsilon(2)$ and $\varepsilon^{\prime}(3)=\varepsilon(3)$. The fact that each and every single term in $\left(B r u c e^{\prime}\right)$ vanishes when $1-x_{1} x_{2}=0$ and $1-\bar{x}_{2} x_{3}=0$ follows from the next $(s u b)^{4}$ lemma:

Subsubsubsublemma 1.5.2.2.1: For each signed permutation $\left(\sigma, \varepsilon^{\prime}\right)$ of $W\left(\mathcal{B}_{3}\right)$,

$$
\left(\sigma, \varepsilon^{\prime}\right)\left[\left(1-x_{1} \bar{x}_{2}\right)\left(1-\bar{x}_{1} \bar{x}_{2}\right)\left(1-x_{1} \bar{x}_{3}\right)\left(1-\bar{x}_{1} \bar{x}_{3}\right)\left(1-x_{2} \bar{x}_{3}\right)\left(1-\bar{x}_{2} \bar{x}_{3}\right)\right]
$$

vanishes when $x_{3}=\left(\bar{x}_{2}\right)^{-1}$ and $x_{1}=\left(x_{2}\right)^{-1}$.

[ Type 'S15221() :' in ROBBINS, to get a rigorous proof.]

\section{Proof:}

It follows that indeed each and every single term of $($ Bruce $)$ vanishes when $x_{3}=\left(\bar{x}_{2}\right)^{-1}$ and $x_{1}=\left(x_{2}\right)^{-1}$, and since $\left(2^{k} k !\right) \cdot 0=0$, the whole thing vanishes as well. This completes the proof of subsubsublemma 1.5.2.2.

Going back to the proof of subsublemma 1.5.2, in order to complete the proof of (Dominique), we need to compare at one more value of $\bar{x}_{2}$. The value to pick is $\bar{x}_{2}=0$, which implies $x_{1}=x_{2}=1$. And, indeed $\Omega_{k}\left(1,1, x_{3}, \ldots, x_{k}\right)=\Omega_{k-2}\left(x_{3}, \ldots, x_{k}\right)$, as follows from $(\overline{\text { Walt }})$ in subsublemma 1.5.2.3 below, applied twice.

Since both sides of (Dominique) are polynomials of degree $\leq 2(k-2)$ in $\bar{x}_{2}$, that agree in $2 k-3$ distinct values of $\bar{x}_{2}$, they must be identically equal. This completes the proof of (Dominique) and hence of $(1.5 .2 a-b)$. 
By symmetry, the statement of (1.5.2c) is equivalent to (Walt) in the following subsubsublemma.

Subsubsublemma 1.5.2.3: $\Omega_{k}(x)=\Phi_{k}(x) / \Delta_{k}(x)(k \geq 1)$ satisfies:

$$
\begin{aligned}
& \Omega_{k}\left(x_{1}, \ldots, x_{k-1}, 0\right)=\Omega_{k-1}\left(x_{1}, \ldots, x_{k-1}\right) . \\
& \Omega_{k}\left(x_{1}, \ldots, x_{k-1}, 1\right)=\Omega_{k-1}\left(x_{1}, \ldots, x_{k-1}\right) .
\end{aligned}
$$

[ Type 'S1523(k):' in ROBBINS, for specific k.]

Proof: By the $W\left(\mathcal{B}_{k}\right)$-symmetry of $\Omega_{k}$ it suffices to prove $(W a l t)$. When $x_{k}=0$ all the summands, in the definition $\left(G_{o g}\right)$ of $\Phi_{k}(x)$, vanish except those coming from $(\pi, \varepsilon)$ for which $\pi(k)=k$, $\varepsilon_{k}=-1$. Thus the sum reduces to a sum over $W\left(\mathcal{B}_{k-1}\right)$ that is easily seen to be equal to

$$
\frac{\Delta_{k}\left(x_{1}, \ldots, x_{k-1}, 0\right)}{\Delta_{k-1}\left(x_{1}, \ldots, x_{k-1}\right)} \Phi_{k-1}\left(x_{1}, \ldots, x_{k-1}\right)
$$

This completes the proof of subsubsublemma 1.5.2.3.

This completes the proof of subsublemma 1.5.2 .

Combining subsublemmas 1.5.1 and 1.5.2, and using induction on $k$, we see that indeed $L_{k}(x)=$ $\Omega_{k}(x)^{2}$, for any $k \geq 2$, since this is true for $k=0,1$. This completes the proof of sublemma 1.5. Combining Sublemmas 1.1"', 1.2"' , and 1.5 finishes the proof of Lemma 1. 


\section{REFERENCES}

[A1] G.E. Andrews, Plane Partitions III: the weak Macdonald conjecture, Invent. Math. 53(1979), 193-225.

[A2] G.E. Andrews, Plane Partitions V: the T.S.S.C.P.P conjecture, J. Combin. Theory Ser. A, 66(1994), 28-39.

[D] R.J. Duffin, Basic properties of discrete analytic functions, Duke Math. J. 23(1956), 335-363.

[G] I. Gessel, Tournaments and Vandermonde's determinant, J. Graph Theory 3(1979), 305-307.

[Ma] I.G. Macdonald, SYMMETRIC FUNCTIONS AND HALL POLYNOMIALS , Oxford University Press, Oxford, 1979.

[MRR1] W.H. Mills, D.P. Robbins, and H.Rumsey, Proof of the Macdonald conjecture, Invent. Math. 66(1982), 73-87.

[MRR2] W.H. Mills, D.P. Robbins, and H.Rumsey, Alternating sign matrices and descending plane partitions, J. Combin. Theo. Ser. A, 34(1983), 340-359.

[MRR3] W.H. Mills, D.P. Robbins, and H.Rumsey, Self complementary totally symmetric plane partitions, J. Combin. Theory Ser. A 42(1986), 277-292.

[PS] G. Pólya and G. Szëgo, PROBLEMS AND THEOREMS IN ANALYSIS[1], Springer, 1976.

[R] D.P. Robbins, The story of 1, 2, 7, 42, 429, 7436, ... , Math. Intell v.13, \#2(Spring 1991), $12-19$.

[RR] D.P. Robbins and H. Rumsey, Jr., Determinants and alternating sign matrices, Advances in Mathematics 62(1986), 169-184.

[Stanl] R.P. Stanley A baker's dozen of conjectures concerning plane partitions, in: COMBINATOIRE ENUMERATIVE, ed. by G. Labelle and P. Leroux, Lecture Notes in Mathematics 1234, Springer, Berlin, 1986.

[Stant] D. Stanton, Sign variations of the Macdonald identities, SIAM J. Math. Anal. 17(1986), 1454-1460.

[Ste] J. Stembridge, A short proof of Macdonald's conjecture for the root system of type A, Proc. Amer. Math. Soc. 102(1988), 777-786.

[WZ] H.S. Wilf and D. Zeilberger, An algorithmic proof theory for hypergeometric (ordinary and "q") multisum/integral identities, Invent. Math. 108(1992), 575-633.

[Z1] D. Zeilberger, The algebra of linear partial difference operators and its applications, SIAM J. 
Math. Anal. 11(1980), 919-934.

[Z2] D. Zeilberger Partial difference equations in $m_{1} \geq \ldots \geq m_{n} \geq 0$ and their applications to combinatorics, Discrete Math 31(1980), 65-77.

[Z3] D. Zeilberger, A constant term identity featuring the ubiquitous (and mysterious) AndrewsMills-Robbins-Rumsey numbers $\{1,2,7,42,429, \ldots\}$, J. Combin. Theory Ser. A 66(1994), 17-27.

[Z4] D. Zeilberger, A unified approach to Macdonald's root-system conjectures, SIAM J. Math. Anal. 19(1988), 987-1013.

[Z5] D. Zeilberger, A Stembridge-Stanton style proof of the Habsieger-Kadell q-Morris identity , Discrete Math. 79(1989/90), 313-322.

[ZB] D. Zeilberger and D.M. Bressoud, A proof of Andrews' q-Dyson conjecture, Discrete Math. 54(1985) , 201-224.

Original Version: Kislev 5753; This Version: Nisan 5755. 


\section{EXODION}

\section{The Skeleton of the Proof with Checkers' Names}

A person's name after the appropriate $(s u b)^{i}$ lemma $(0 \leq i \leq 6)$, means that she or he has checked that its proof is formally correct, provided that all the direct descendent-sublemmas are correct. They assume no responsibility whatsoever to the actual correctness of the offsprings of the $(s u b)^{i}$ lemma that they have been charged with, that lies solely with their subcheckers.

1 (G. Andrews, D. Foata, W. Johnson)

1.1 (N. Alon, H. Wilf)

1.1.1 (A. Björner)

1.1.1.1 (C. Orr)

1.1.1.2 (D. Bar-Natan)

1.1.1.2.1 (J. Noonan)

1.1.1.2.2 (J. Majewicz)

1.1.1.3 (S. Cooper)

$1.1 .2(\mathrm{~V} . \mathrm{Kann})$

1.1.3 (R. Stanley)

1.2 (X.Y. Sun)

1.2.1 (B. Salvy)

1.2.1.1 (D. Stanton)

1.2.1.2 (Anon., J. Legrange)

1.2.1.2.1 (N. Bergeron)

1.2.1.2.1.1 (R. Simion, R.J. Simpson)

1.2.1.2.1.1.1 (R. Howe, R. Graham)

1.2.1.2.1.1.2 (J. Borwein, D.E. Knuth)

1.2.1.2.2 (D. Robbins)

1.2.1.2.3 (L. Zhang) 


\subsection{1 (M. Bousquet-Mélou)}

1.2.1.2.3.1.1 (L. Habsieger) (also requires 1.4.1.1)

1.2.1.2.4 (F. Brenti)

1.2.1.2.5 (L. Ehrenpreis, S.B. Ekhad, G. -C. Rota)

1.2.1.3 (F. Bergeron, M. Wachs)

1.3 (M. Knopp, S. Parnes)

1.3.1 (M. Knopp)

1.3.1.1 (R. Canfield)

1.3.1.1.1 (W. Chen)

1.3.1.2 (J. Noonan)

1.3.1.3 (K. Ding)

1.3.1.4 (P. Zimmermann)

Case I (C. Dunkl, R. Ehrenborg)

Subcase Ia (C. Dunkl)

Subcase Ib (C. Dunkl, K. Eriksson, R. Sulanke)

Case II (M. Werman)

1.4 (O. Foda)

1.4.1 (J. Haglund)

1.4.1.1 (M. Readdy, N. Takayama)

1.4.1.1.1 (A. Fraenkel, J. Labelle) (also requires section 1.5.2)

1.4.1.2 (J. Friedman)

1.4.1.3 (S. Okada, Chu W.)

1.4.1.4 (F. Garvan, G. Gasper)

Case I (V. Strehl)

Subcase Ia (A. Granville) 
Subcase Ib (E. Grinberg)

Case II (G. Kalai, I. Sheftel)

1.4.1.4.1 (C. Krattenthaler)

1.4.1.4.1.1 (G. Labelle)

1.4.1.5 (G. Almkvist, D. Loeb, V. Strehl)

Case I (P. Leroux, V. Strehl)

Subcase Ia (E. Lewis, V. Strehl)

Subcase Ib (D. Loeb, V. Strehl)

Case II (V. Strehl)

1.4.1.5.1 (G. Bhatnagar, S. Milne, V. Strehl)

1.4.1.6 (F. Garvan)

1.5 (R. Supper)

1.5.1 (Han G.-N., P. Paule, A. Ram)

1.5.1.1 (S. B. Ekhad, C. Zeilberger)

1.5.1.2 (S. B. Ekhad, T. Zeilberger)

1.5.2 (A. Regev, J. Remmel)

1.5.2.1 (C. Reutenauer, B. Reznick)

Case I (B. Reznick)

Case II (B. Reznick, C. Rousseau)

1.5.2.2 (B. Sagan)

1.5.2.2.1 (S.B. Ekhad, H. Zeilberger)

1.5.2.3 (W. Stromquist, X.G. Viennot) 


\section{The Checkers}

The sublemmas checked by each of the checkers is given inside the square bracket at the end of each entry, where the periods were dropped. Thus [123456] would stand (if it existed) for 1.2.3.4.5.6. If the person also contributed general comments about the rest of the paper, this is indicated by 'GC'. Persons who are only credited with 'GC' contributed important general comments, but did not check the formal correctness of any statement.

The following abbreviations were used: AcM:='Acta Math.', AdM:='Advances in Math', AMM:='Amer. Math. Monthly', BAMS:='Bulletin of the Amer. Math. Soc', CM:='Contemporary Math.', CR:='Comptes Rendus', DM:='Discrete Math.', JCT(A):='J. of Comb. Theory (Ser. A)', ICM:='Inter. Congress of Mathematicians', JSC:='J. for Symbolic Comp.', LNM:='Lecture Notes in Math'(Springer), MI:='Math. Intell.', PNAS:='Proc. Nat. Acad. Sci.', PAMS:='Proc. Amer. Math. Soc.', Inv:='Inventionae Math.', SB:='Seminaire Bourbaki',. Sloane's abbreviated citing scheme has been followed: [volume first_page year].

Gert Almkvist, Lund, gert@maths.1th.se, is the 'guy who generalized a mistake of Bourbaki' (CM 143609 93), which lead to important work in algebraic K-theory. Now he became a wizard in asymptotic number theory (CM $1432193)$. [1415]

Noga Alon, Tel-Aviv, noga@math.tau.ac.il , is one of the most powerful combinatorialists, graph-theorists, and complexity-ists in the world, who developed and applied innovative algebraic techniques. His many honors include ICM 90', and being granted a permanent-member-size office when he was member of IAS in 93-94. [11]

George Andrews, Penn State, andrews@math.psu.edu, is the king of q-theory, who pioneered computer-assisted research that lead to astounding results in combinatorics, number theory, and physics (e.g. CBMS \#66,AMS.) [1]

Richard Askey, Wisconsin, askey@math.wisc.edu, is the king of special functions, of Askey-Gasper inequality (AcM 154137 85) and Askey-Wilson polynomials fame. [GC]

Dror Bar-Natan, Harvard, dror@math.harvard.edu, has done ground-breaking work in topological Quantum Field theory, and has a novel approach to Vassiliev invariants. His beautiful papers can be anonymously ftp-ed from math.harvard.edu, and once he moves to Jerusalem (Sept. 1995), from math.huji.ac.il. [1112]

Bernard Beauzamy, Paris VII and Inst. Calc. Math., beauzamy@mathp7.jussieu.fr , is a leading Banach spacer who has also made significant contributions to computer algebra (JSC 13463 92.)[GC]

Francois Bergeron, UQAM, bergeron@catalan.math.uqam.ca, is a leading Species-ist who has also made beautiful work on card shuffling and the descent algebra. [1213]

Nantel Bergeron, Harvard, nantel@math.harvard.edu, contributed significantly to card-shuffling, knot theory (with Bar-Natan), the descent algebra, and Schubert polynomials.[12121]

Gaurav Bhatnagar, Ohio-State, gaurav@math.ohio-state.edu, is completing a deep and important thesis under Steve Milne. [14151]

Anders Björner, Stockholm, bjorner@math.kth.se, is a leading algebraic combinatorialist (e.g. ICM 86), who has 
applied the arcane Möbuius function to mundane, but important, computational complexity problems. [111]

Jonathan Borwein, Simon Fraser, jborwein@cecm.sfu.ca, The Borwein brothers showed the world (with the Chudnowsky brothers) that computing $\pi$ to zillion digits involves very beautiful and deep mathematics. Their paper (with Dilcher) (AMM 100274 93), according to Andrews(MI 16\#4 16 94), is a prime example of why humans will never be replaceable by machines. [1212112]

Mireille Bousquest-Mélou, bousquet@labri.u-bordeaux.fr, has come a long way since she was the 'first woman to have ever ranked first in the concourse to the 'great' schools of France.' She has used Viennot's heaps, as well as the DSV methodology, to find very deep, and elegant, results in polyomino-enumeration. (Publ. LACIM (UQAM), \#8). [121231]

Francesco Brenti, Perugia and IAS, brenti@math.ias.edu, applied total positivity to prove deep combinatorial inequalities. Currently he is studying the Kazhdan-Lusztig polynomials from a combinatorial point of view. [12124]

David M. Bressoud, Macalister, dvb@math.psu.edu, is a leading q-ist (JNT 1287 80) and combinatorialist(DM 38313 82), who is also a master expositor('Second Year Calculus', 'Radical Calculus'.) [So far everything except for 13 and 14 and their descendents.]

E. Rodney Canfield, Georgia, erc@csun1.cs.uga.edu , disproved the Rota conjecture (AdM 29 179 ), and did many more things since, especially in asymptotic combinatorics. [1311]

William Chen, LANL, chen@monsoon.c3.lanl.gov, did breathtaking work on bijections(PNAS 87 9635 90), formal grammars, permutation statistics and many other things. [13111]

Chu Wenchang, Rome, wenchang@mat.uniroma1.it, has done beautiful work in systematizing and explaining combinatorial identities. [1413]

Shaun Cooper, Wisconsin, scooper@math.wisc.edu, is currently completing his thesis under Dick Askey, and has proved a conjecture of P. Forrester (DM, to appear.) [1113]

Kequan Ding, IAS and Illinois, ding@math.ias.edu has done beautiful work on the crossroads of combinatorics, geometry, and topology. His approach to the Rook polynomials is wonderful. [1313]

Charles Dunkl, Virginia, cfd5z@virginia.edu, is of 'Dunkl operators' fame, without which Cherednick would not have been able to prove Macdonald's constant term conjecture. [1314, case I, and descendents]

Richard Ehrenborg, ehrenborg@mipsmath.math.uqam.ca, is both a great juggling mathematician and a great mathematical juggler.[1314, case I, and descendents]

Leon Ehrenpreis, Temple, leon@math.temple.edu, did work on partial differential equations that Halmos et. al. (AMM 83503 76) consider to be one of the crowning achievements of American mathematics since 1940. He is listed by Dieudonné ('Panorama of Pure Math', p. 70,) along with Laplace, Fourier, and 13 others, as one of the originators of the general theory of linear partial differential equations. [12125,GC] 
Shalosh B. Ekhad, Temple, ekhad@math.temple.edu, has written more than fifteen papers, and intends to write many more. [12125,1511,1512,15221]

Kimmo Eriksson, KTH, kimmo@math.kth.se has done beautiful work on Coxeter groups, the Number game, and Fulton's essential set. He is also a great speaker. [1314, subcase Ib]

Dominique Foata, Strasbourg, foata@math.u-strasbg.fr, has revolutionized combinatorics at least twice (LNM \#85 and JCT(A) 2425078 (see also ICM 83)). The first time it also revolutionized theoretical computer science, and the second time it also revolutionized special functions. [1]

Omar Foda, Melbroune, foda@maths.mu.0Z.AU, is a leading mathematical physicist and quantum group-ist, who has proved far-reaching Rogers-Ramanujan-type identities.[14]

Aviezri Fraenkel, Weizmann Inst., MAFRAENKEL@WEIZMANN. WEIZMANN. AC. IL, has both Erdös number 1, and Einstein number 2 (via his advisor Ernst Strauss.) He is a leader in games, combinatorial number theory, complexity, and many other things. His uncle is Abraham H. of Zermelo-F. fame. [14111]

Jane Friedman, janef@teetot. acusd.edu, has made lasting contributions to Rogers-Ramanujan theory (Temple thesis, 90), to the generalized Odlyzko conjecture (PAMS 1181013 93), and to lattice path counting. [1412]

Frank Garvan, Florida, frank@math.ufl.edu, cracked a 1944-conjecture of Dyson by cranking out a new 'crank' for partitions (Dyson, in: 'Ramanujan Revisited'). He also did many other things, including the first proof of the F4-Macdonald root system conjecture (BAMS 24343 91). [1416]

George Gasper, Northwestern, george@math.nwu.edu, is of the fame of the Askey-Gasper inequality and of the Gasper-Rahman classic on 'Basic Hypergeometric Series'. He is the most serious candidate for proving an inequality that was shown by Jensen to imply RH. [1414]

Bill Gosper, Independent, rwg@NEWTON.macsyma.com, is a 20th-century Ramanujan. His Gosper algorithm (PNAS 754078 ) changed summation theory for ever. [GC]

Ron Graham, Bell, rlg@research.att.com, ( $\in$ NAS), is present and former president of AMS and AJA resp. Both his technical contributions and charismatic personality (e.g. DLS II, Univ. Videos) has helped make combinatorics a house-hold name. [1212111]

Andrew Granville, Georgia, andrew@sophie.math.uga.edu , proved the Carmichael conjecture (with Pomerance)(ICM 94), need I say more? [1414, subcase Ia]

Eric Grinberg, Temple, grinberg@math.temple.edu , has done important work on the Radon transform, and on the Bussman-Petit problem. [1414, subcase Ib]

Laurent Habsieger, Bordeaux, habsieger@geocub.greco-prog.fr, proved, in his brilliant thesis, Askey's conjectured extension of Selberg's integral (also done by Kadell), and the G2 case of Macdonald's constant term conjecture (CR 303211 86), (also done by Zeilberger.) He has since diversified, and did beautiful work in number theory. Most recently he improved bounds in the notorious 'football(soccer) pool' problem. [1212311] 
Jim Haglund, Illinois (starting 8/95), until 8/95: jhaglund@kscmail. Kennesaw.edu, has written a brilliant thesis under Rodney Canfield, that contains lots of beautiful and deep results about rook polynomials, KOH-type identities, and the Simon Newcomb problem. Lately he is studying the combinatorics of the Riemann zeta function. [1414, case I]

Han Guo-Niu, Strasbourg, han@math.u-strasbg.fr , is the world's greatest expert on the Denert statistics, and is a very powerful bijectionist and word-enumerator. [151]

Roger Howe, Yale, howe@math.yale.edu, $(\in \mathrm{NAS})$, is not only one of the deepest and most influential Lie theorists of our day, but also a great lecturer and expositor (AMM 9035383 (L. Ford 84' award.)) [1212111]

Warren Johnsnon, Penn State, johnson@math.psu.edu, has done deep and elegant work both on the q-Bell numbers and q-Abel identities. [1,GC]

Gil Kalai, Jerusalem, kalai@cs.huji.ac.il, revolutionized convexity and polytope theory with his 'algebraic shifting' (ICM 94). With Jeff Kahn, he finished off (negatively) the famous Borsuk problem (that resisted the attacks of at least one Fields-medalist). [1414, case II]

Viggo Kann, KTH, viggo@nada.kth.se, wrote a brilliant thesis on approximate algorithms. His computer, Sol Tre, is a co-author of S.B. Ekhad. [112]

Marvin Knopp, Temple, Never had e-mail (and never will), wrote the classic 'Modular Functions' (Chelsea), started the notion of 'rational period function' and developed a deep theory of Eichler cohomology. [13,131]

Don Knuth, Stanford, No more e-mail, ( $\in$ NAS), is both the Diderot (Art of Comp. Prog.), and Gutenberg(TeX) of the computer age. He is the unique element of TURING $\cap$ HOPPER. [1212112,GC]

Christian Krattenthaler, Vienna, kratt@pap.univie.ac.at , is a Maestro in piano playing, lattice-path combinatorics, hypergeometric series, and computer algebra. [14141]

Gilbert Labelle,UQAM, gilbert@mipsmath.math.uqam.ca, of Species-fame, has the nicest proof ever, of the Largange inversion formula (AdM 42217 81.) [141411]

Jacques Labelle,UQAM, jacques@mipsmath.math.uqam.ca , is a master chess player, species-ist and combinatorialist. [14111]

Jeff Lagarias, Bell, jcl@research.att.com , is not only a high-powered researcher in number theory, cryptology and complexity theory, but is also a master expositor (AMM 923 85, L. Ford award 86'), with a deep sense of history. With Shor he disproved a long-standing conjecture of Keller about tiling (BAMS 27 279 92). This leads me to believe that perhaps the Jacobian conjecture (also posed by Keller) is false as well, at least in high dimensions. [GC]

Jane Legrange, Bell, jdl@allwise.att.com , is a Member of Technical Staff in the Display Research Department, at AT\&T Bell Labs, Murray Hill, NJ. Thanks to people like her, very soon we won't need paper or printers. In 19921993, she was Visiting Associate Professor at Princeton University, as a recipient of the coveted VPW NSF grant. 
She published extensively on liquid crystals and DNA, in numerous journals, including Physical Review Letters and Nature. [1212, GC]

Pierre Leroux, UQAM, leroux@mipsmath.math.uqam.ca, did beautiful work both on species and (with Viennot) on the combinatorics of differential equations. With Foata, he has met Askey's challenge: to find a combinatorial proof to the generating function for Jacobi polynomials. (PAMS 8747 83.) [1415, case I]

Ethan Lewis, Haverford, e1lewis@haverford.edu, proved a long-standing conjecture on infinite covering systems, and extended the Cartier-Foata method. [1415, subcase Ia]

Daniel Loeb, Bordeaux, labri@u-bordeaux.fr, has (in part with Rota) largely extended the scope of the Umbral Calculus, to include formal power series of logarithmic type. He is a master of 'negative thinking' and has started a new era in the umbral calculus [1415,subcase Ib]

John Majewicz, Temple, jmaj@math.temple.edu, is currently studying for his Ph.D. under D. Zeilberger. He has extended the WZ method to Abel-type sums. His papers can be anon. ftp-ed from ftp.math.temple.edu, directory pub/jmaj. [11121][11122]

Steve Milne, Ohio-State, milne@function.mps.ohio-state.edu, does extremely deep research in multivariate hypergeometric series. One of Gelfand's dreams is to relate his geometro-combinatorial approach to hypergeometric functions with Milne's (and Gustafson's) work. [14151]

John Noonan, Temple, noonan@math.temple.edu, is currently studying for his Ph.D. under D. Zeilberger. He studies permutations that sin a prescribed number of times. His great programs and papers can be downloaded from http://www.math.temple.edu/ ${ }^{\sim}$ noonan. [11121]

Andrew Odlyzko, Bell, amo@research.att.com. Contrary to what Herb Wilf (and probably many others) used to believe, ODLYZKO is not a collective name (like Bourbaki) for a team of brilliant mathematicians, but consists of a singleton. His many contributions include work on small discriminants, the disproof of the Mertens conjecture, and vastly extending the empirical evidence for RH (ICM 86). [GC]

Kathy O'Hara, Independent (formerly Iowa),ohara@math.uiowa.edu, has astounded the combinatorial world with her injective proof of the unimodality of the Gaussian polynomials (AMM 96590 89.) [1212111,GC]

Soichi Okada, Nagoya,okada@nagoya-u.ac.jp, is one of the world's greatest determinant and pfaffian evaluators, and an all-around algebraic combinatorialist. [1413]

Craig Orr, Miami, craig@paris-gw.cs.miami.edu , has completed a brilliant thesis under D. Zeilberger. [1111]

Sheldon Parnes, Simon Fraser, sparnes@cecm.sfu.ca, completed last year a brilliant thesis under D. Zeilberger. [13]

Peter Paule, RISC-Linz, ppaule@risc.uni-linz.ac .at, is at the cutting edge between combinatorics and computer algebra. He contributed significantly to the Bailey chain, developed a general theory of summation, and found short and sweet computer proofs (much shorter then the original ones by Ekhad and Tre) of the Rogers-Ramanujan 
identities (ElJC 1 R10.) [151, 152, and their descendents]

Bob Proctor, North Carolina, rap@math.unc.edu, interfaces the combinatorics of tableaux with representation theory very elegantly. His beautiful exposition (AMM 89721 82) of Stanley's brilliant proof of the Erdös-Moser conjecture, stripped it of its inessential (and intimidating) high-brow components, and exposed its bare beauty for the rest of us to savor. [13111]

Arun Ram, Wisconsin, ram@math.wisc.edu, has a very healthy schizophrenia between combinatorics and representation theory, that helped both very much. He is also a brilliant lecturer. [151]

Marge Readdy, readdy@catalan.math.uqam.ca, is one of the most brilliant academic grandchildren of Richard Stanley. She is a very versatile posetist. [1411, GC]

Jeff Remmel, UCSD, jremmel@math.ucsd.edu , has done elegant and deep work on tableaux, permutations, and many other combinatorial objects. The Garsia-Remmel rook polynomials attracted great attention. [152]

Amitai Regev, Penn State and Weizmann Inst., regev@math.psu.edu, proved, in his thesis, that the tensor product of PI rings is yet another one. He is of 'Regev theory' fame (see Rowan's book) that uses tableaux to study PI algebras. [152]

Christoph Reutenauer, UQAM, christo@catalan.math.uqam.ca, has a way with words and plays freely with Lie algebras. Read all about it in the proc. of FPSAC VI. [1521]

Bruce Reznick, Illinois, reznick@math.uiuc.edu, is one of the greatest 19th-century algebraists alive. By the periodicity of mathematical fashions, he is also a great 21st-century mathematician. Hilbert, would have loved his work. [1521]

Don Richards, Virginia, dsr2j@virginia.edu, is a leader on complex functions of matrix arguments. With Ken Gross, he is developing a deep theory of hypergeometric functions on complex matrix space (BAMS 24 349 91.) [GC]

Dave Robbins, IDA-CRD, robbins\%idacrd@princeton.edu, is responsible (with WM and HR), to my spending (wasting(?)) a big chunk of my life proving (which was fun) and then debugging (which was no fun!) the ASM conjecture. Before coming up with his notorious conjectures, he proved his mettle by proving the 'most interesting open problem in enumeration' (Stanley): Macdonald's formula for CSPPs (ref. [MRR1].) [12122]

Gian-Carlo Rota, MIT, rota@math.mit.edu, $(\in$ NAS), has laid the foundation to Combinatorial Theory (see 'Classic Papers in Comb.', ed. by Gessel and Rota.) [12125]

Cecil Rousseau, Memphis State, rousseau@hermes.msci.memst.edu is a great graph theorist and problem solver. He served as coach of the US math Olympic team for many years. [1521, case II]

Bruce Sagan, Michigan State, sagan@nsf1.mth.msu.edu , is a great bijectionist and master expositor ('The symmetric group'). [1522] 
Bruno Salvy, INRIA, Bruno.Salvy@inria.fr, is pioneering the use of computer algebra for combinatorics and theoretical computer science. With Paul Zimermann, he has developed the versatile Maple package gfun for handling generating functions.[121]

Isabel Sheftel, Jerusalem, sheftel@cs.huji.ac.il, is writing a thesis under the direction of Gil Kalai. [1414, case II]

Rodica Simion, George Washington, simion@gwuvm.gwu.edu, has done very original and seminal work both on bijections, posets, enumeration, and (multi-q) theory. [121211]

R. Jamie Simpson, Curtin, TSIMPSONR@cc.curtin.edu.au, is one to the leaders in covering sequences. He had a beautiful (and hence elementary) proof (found independently by Berger, Felzenbaum, and Fraenkel) that the top moduli of an exact covering set must be equal. [121211]

Richard Stanley, MIT, rstan@math.mit.edu, is perhaps more responsible than anyone else to the creation of a new AMS classification (05E.) The totality of his influence is even more than the sum of its parts, which include having so many brilliant academic offsprings, some of whom are listed here. [113]

Dennis Stanton, Minnesota, stanton@s2.math.umn.edu , is equally comfortable with block designs (from a fancy, 'algebraic' point of view.), bijections ('Constructive combinatorics', with D. White), and hypergeometric analysis, to all of which he contributed deeply. Last but not least he is co-responsible for the 'Stanton-Stembridge trick'. [1211]

Volker Strehl, strehl@immd1.informatik.uni-erlangen.de, has carried the Foata approach to special functions to breathtaking heights. His Habilitationschrift is a true classic, that I hope would be published as a book. [1414, case I; 1415 and its descendents]

Walt Stromquist, Wagner Assoc., 74730.2425@compuserve.com, was, for a few months in 1975, the holder of the record for the minimal number of countries needed for a planar map not to be four-colorable. He retains this title, to this day, amongst humans. Since then he did a lot of things, including instructions on how to divide a cake fairly. [1523]

Bob Sulanke, Boise State, sulanke@math.idbsu.edu, did beautiful and elegant work on the combinatorics of lattice paths. He is a master of elegant bijections. [1314, subcase Ib]

X.Y.Sun, Temple, xysun@math.temple.edu, hopes to start his dissertation work soon, under D. Zeilberger. He is a true Maple whiz. [12]

Sheila Sundaram, Miami, sheila@paris-gw.cs.miami.edu, is a leading symplectic tableau-ist and plethysm-ist. See FPSAC VI. [GC]

Raphaële Supper, Strasbourg, c/o avanissian@math.u-strasbg.fr, is a very promising young analyst, who has already contributed significantly to the theory of entire arithmetic functions (CR 312781 91,) and to harmonic functions of exponential type. [15]

Nobuki Takayama, Kobe, taka@math.s.kobe-u.ac.jp, is one of the greatest Gröbner bases algorithmicians in the 
world, as applied to special functions. He developed the package KAN. [1411]

Xavier G. Viennot, Bordeaux, viennot@geocub.greco-prog.fr revolutionized combinatorial statistical physics (SB, Ex. \# 626). His charismatic research and lecture-persona ('Viennotique'= transparency wizardry,) helped make combinatorics mainstream, even in (formerly) snobbish France. He won a CNRS silver medal. [1523,GC]

Michelle Wachs, Miami, wachs@math.miami.edu , is a leading algebraic combinatorialist. She worked on posets, permutation statistics, and vastly extended the scope of shellabilty, that, according to Gil Kalai, should from now on be called michelle-ability. [1213]

Michael Werman, Jerusalem, werman@cs.huji.ac.il, is a master (mathematical-) imager (e.g. PRL 5 87 207). $[1314$, case II]

Herb Wilf, Penn, wilf@central.cis.upenn.edu, went through the three analyses: numerical, mathematical, and (happily ever after,) combinatorial. With Nijenhuis and Greene he designed the best mathematical proof ever. He was a pioneer in combinatorial algorithms, in particular for random selection and listings of combinatorial objects. (See CBMS \#55, SIAM.) [11]

Celia Zeilberger, 71324.3565@compuserve.com, attends sixth grade at John Witherspoon Middle School, Princeton, NJ. [1511]

Hadas Zeilberger, 71324.3565@compuserve.com, attends 'A-class' at the U-NOW Day Nursery, Princeton, that is located on the same street (a couple of blocks away) from the residence of the (tentative) prover of FLT. [15221]

Tamar Zeilberger, 71324.3565@compuserve.com, attends third grade at Johnson Park Elementary School, Princeton, NJ. [1512]

Li Zhang, Temple, lzhang@math.temple.edu, is working towards his dissertation, under D. Zeilberger. He is a great Maple whiz. [12123]

Paul Zimmermann, INRIA, Paul.Zimmermann@inria.fr, is one of the pioneers in the use of computer alegbra for combinatorics and theoretical computer science. See also under Salvy.[1314] 